This is a self-archived version of an original article. This version may differ from the original in pagination and typographic details.

Author(s): Don, Sebastiano; Vittone, Davide

Title: Fine properties of functions with bounded variation in Carnot-Carathéodory spaces

Year: 2019

Version: Accepted version (Final draft)

Copyright: (c) 2019 Elsevier Inc.

Rights: $C C B Y-N C-N D 4.0$

Rights url: https://creativecommons.org/licenses/by-nc-nd/4.0/

Please cite the original version:

Don, S., \& Vittone, D. (2019). Fine properties of functions with bounded variation in CarnotCarathéodory spaces. Journal of Mathematical Analysis and Applications, 479(1), 482-530. https://doi.org/10.1016/j.jmaa.2019.06.035 


\title{
FINE PROPERTIES OF FUNCTIONS WITH BOUNDED VARIATION IN CARNOT-CARATHÉODORY SPACES
}

\author{
SEBASTIANO DON AND DAVIDE VITTONE
}

\begin{abstract}
We study properties of functions with bounded variation in Carnot-Carathéodory spaces. We prove their almost everywhere approximate differentiability and we examine their approximate discontinuity set and the decomposition of their distributional derivatives. Under an additional assumption on the space, called property $\mathcal{R}$, we show that almost all approximate discontinuities are of jump type and we study a representation formula for the jump part of the derivative.
\end{abstract}

\section{INTRODUCTION}

A lot of effort was devoted in the last decades to the development of Analysis and Geometry in general metric spaces and, in particular, to the study of functions with bounded variation $(B V)$. Carnot-Carathéodory $(C C)$ spaces are among the most fruitful settings where $B V$ functions have been introduced $([10,20])$, see also [8, 12, [19, 22, 23, 24] and the more recent [3, 5, 6, 9, 11, 15, 31, 35, 44. The aim of this paper is to give some contributions to this research lines by establishing "fine" properties of $B V$ functions in CC spaces. A non-trivial part of our work consists in fixing the appropriate language in a consistent and robust manner.

A CC space is the space $\mathbb{R}^{n}$ endowed with the Carnot-Carathéodory distance $d$ (see (10) arising from a fixed family $X=\left(X_{1}, \ldots, X_{m}\right)$ of smooth, linearly independent vector fields (called horizontal) in $\mathbb{R}^{n}$ satisfying the Hörmander condition, see (2). As customary in the literature, we always assume that metric balls are bounded with respect to the Euclidean topology. Moreover, we work in equiregular CC spaces, where a homogeneous dimension $Q$, usually larger than the topological dimension $n$, can be defined; recall that any CC space can be lifted to an equiregular one, see e.g. [42].

The space $B V_{X}$ of function with bounded $X$-variation consists of those functions $u$ whose derivatives $X_{1} u, \ldots, X_{m} u$ in the sense of distributions are represented by a vector-valued measure $D_{X} u$ with finite total variation $\left|D_{X} u\right|$. These functions have been extensively studied in the literature and important properties have been proved, like coarea formulae, approximation theorems, Poincaré inequalities.

We now describe some of the results we prove in this paper. The first one, Theorem 1.1 below, concerns the almost everywhere approximate $X$-differentiability (see Section 2.3) of $B V_{X}$ functions; its classical counterpart is very well-known, see e.g. [2, Theorem 3.83]. As customary, we denote by $D_{X}^{a} u$ and $D_{X}^{s} u$, respectively, the absolutely continuous and singular part of $D_{X} u$ with respect to the Lebesgue measure $\mathscr{L}^{n}$.

Date: September 24, 2018.

2010 Mathematics Subject Classification. 26B30, 53C17, 49Q15, $28 \mathrm{~A} 75$.

Key words and phrases. Functions with bounded variation, Carnot-Carathéodory spaces.

The authors are supported by the University of Padova Project Networking and STARS Project "SubRiemannian Geometry and Geometric Measure Theory Issues: Old and New" (SUGGESTION), and by GNAMPA of INdAM (Italy) project "Campi vettoriali, superfici e perimetri in geometrie singolari". The second named author wishes to ackowledge the support and hospitality of FBK-CIRM (Trento), where part of this paper was written. 
Theorem 1.1. Let $\left(\mathbb{R}^{n}, X\right)$ be an equiregular $C C$ space, let $\Omega \subseteq \mathbb{R}^{n}$ be an open set and let $u \in B V_{X}\left(\Omega ; \mathbb{R}^{k}\right)$. Then $u$ is approximately $X$-differentiable at $\mathscr{L}^{n}$-almost every point of $\Omega$. Moreover, the approximate $X$-gradient coincides $\mathscr{L}^{n}$-almost everywhere with the density of $D_{X}^{a} u$ with respect to $\mathscr{L}^{n}$.

The proof of Theorem 1.1 is based on Lemma 3.12, that is, on a suitable extension to CC spaces of the inequality

$$
\int_{B(p, r)} \frac{|u(q)-u(p)|}{|q-p|} d \mathscr{L}^{n}(q) \leq C \int_{0}^{1} \frac{|D u|(B(p, t r))}{t^{n}} d t
$$

valid for a classical $B V$ function $u$ on $\mathbb{R}^{n}$. Lemma 3.12 answers an open problem stated in [5] and it is new even in Carnot groups. We only recall that Carnot groups are connected, simply connected and nilpotent Lie groups whose Lie algebra is stratified, and we refer to [18, 38, 31, 30. for more detailed introduction to the subject. Carnot groups possess a canonical CC structure obtained by fixing a basis $X_{1}, \ldots, X_{m}$ of the first layer of the Lie algebra of left-invariant vector fields; their importance in the theory stems from the fact that they constitute the infinitesimal models of equiregular CC spaces, a fact that we heavily use in this paper.

Theorem [1.1 was proved in the setting of Carnot groups in [5] together with the following result, which we also extend to our more general setting. We denote by $\mathscr{H}^{Q-1}$ the Hausdorff measure of dimension $Q-1$ and by $\mathcal{S}_{u}$ the set of points where a function $u$ does not possess an approximate limit in the sense of Definition 2.19.

Theorem 1.2. Let $\left(\mathbb{R}^{n}, X\right)$ be an equiregular $C C$ space, let $\Omega \subseteq \mathbb{R}^{n}$ be an open set and let $u \in B V_{X}\left(\Omega ; \mathbb{R}^{k}\right)$. Then $\mathcal{S}_{u}$ is contained in a countable union of sets with finite $\mathscr{H}^{Q-1}$ measure.

In the classical theory, an important object associated with a $B V$ function $u$ is its jump set: roughly speaking, this is the set of points $p$ for which there exist $u^{+}(p) \neq u^{-}(p)$ and a unit direction $\nu_{u}(p)$ such that, for small $r>0, u$ is approximately equal to $u^{+}(p)$ on half of $B(p, r)$ and to $u^{-}(p)$ on the complementary half of $B(p, r)$, the two halves being separated by an hyperplane orthogonal to $\nu_{u}(p)$. In this paper we introduce the notion of approximate $X$-jumps, see Definition 2.24, this requires a certain amount of preliminary work, expecially about "fine" local properties of hypersurfaces with intrinsic $C^{1}$ regularity $\left(C_{X}^{1}\right)$.

We denote by $\mathcal{J}_{u} \subseteq \mathcal{S}_{u}$ the set of $X$-jump points of $u$ and by $\left(u^{+}(p), u^{-}(p), \nu_{u}(p)\right)$ the approximate $X$-jump triple (see Definition 2.24) at a point $p \in \mathcal{J}_{u}$. The measures

$$
D_{X}^{j} u:=D_{X}^{s} u\left\llcorner\mathcal{J}_{u}, \quad D_{X}^{c} u:=D_{X}^{s} u\left\llcorner\left(\Omega \backslash \mathcal{J}_{u}\right),\right.\right.
$$

are called, respectively, jump part and Cantor part of $D_{X} u$. We want to study some further properties of $D_{X} u$ and its decomposition

$$
D_{X} u=D_{X}^{a} u+D_{X}^{s} u=D_{X}^{a} u+D_{X}^{c} u+D_{X}^{j} u .
$$

We state some of them in the following result, which is a consequence of Theorem 3.16 and Proposition 3.3 .

Theorem 1.3. Let $\left(\mathbb{R}^{n}, X\right)$ be an equiregular $C C$ space and consider an open set $\Omega \subseteq \mathbb{R}^{n}$, a function $u \in B V_{X}\left(\Omega ; \mathbb{R}^{k}\right)$ and a Borel set $B \subseteq \Omega$. Then the following facts hold:

(i) there exists $\lambda: \mathbb{R}^{n} \rightarrow(0,+\infty)$ (not depending on $\Omega$ nor $u$ ) locally bounded away from 0 such that $\left|D_{X} u\right| \geq \lambda\left|u^{+}-u^{-}\right| \mathscr{S}^{Q-1}\left\llcorner\mathcal{J}_{u}\right.$

(ii) if $\mathscr{H}^{Q-1}(B)=0$, then $\left|D_{X} u\right|(B)=0$;

(iii) if $\mathscr{H}^{Q-1}(B)<+\infty$ and $B \cap \mathcal{S}_{u}=\emptyset$, then $\left|D_{X} u\right|(B)=0$;

(iv) $D_{X}^{a} u=D_{X} u\left\llcorner(\Omega \backslash S)\right.$ and $D_{X}^{s} u=D_{X} u\llcorner S$, where

$$
S:=\left\{p \in \Omega: \lim _{r \rightarrow 0} \frac{\left|D_{X} u\right|(B(p, r))}{r^{Q}}=+\infty\right\} ;
$$


(v) $\mathcal{J}_{u} \subseteq \Theta_{u}$, where $\Theta_{u} \subseteq S$ is defined by

$$
\Theta_{u}:=\left\{p \in \Omega: \liminf _{r \rightarrow 0} \frac{\left|D_{X} u\right|(B(p, r))}{r^{Q-1}}>0\right\} .
$$

However, for classical $B V$ functions much stronger results than Theorems 1.1 and 1.3 are indeed known: some of them are proved in the present paper also for $B V_{X}$ functions under the additional assumption that the space $\left(\mathbb{R}^{n}, X\right)$ satisfies the following natural condition.

Definition 1.4 (Property $\mathcal{R}$ ). Let $\left(\mathbb{R}^{n}, X\right)$ be an equiregular $\mathrm{CC}$ space with homogeneous dimension $Q$. We say that $\left(\mathbb{R}^{n}, X\right)$ satisfies the property $\mathcal{R}$ if, for every open set $\Omega \subseteq \mathbb{R}^{n}$ and every $E \subseteq \mathbb{R}^{n}$ with locally finite $X$-perimeter in $\Omega$, the essential boundary $\partial^{*} E \cap \Omega$ of $E$ is countably $X$-rectifiable, i.e., there exists a countable family $\left(S_{i}\right)_{i \in \mathbb{N}}$ of $C_{X}^{1}$ hypersurfaces such that $\mathscr{H}^{Q-1}\left(\partial^{*} E \cap \Omega \backslash \cup_{i \in \mathbb{N}} S_{i}\right)=0$.

Recall that a measurable set $E \subseteq \mathbb{R}^{n}$ has locally finite $X$-perimeter in $\Omega$ if its characteristic function $\chi_{E}$ has locally bounded $X$-variation in $\Omega$, while we refer to Definition 2.21 for the essential boundary $\partial^{*} E$. It was proved in the fundamental paper [1] that the $X$-perimeter measure $\left|D_{X} \chi_{E}\right|$ of $E$ can be represented as $\theta \mathscr{H}^{Q-1}\left\llcorner\partial^{*} E\right.$ for a suitable positive function $\theta$ that is locally bounded away from 0 , see Theorem 2.39.

The validity of property $\mathcal{R}$ ("rectifiability") for general equiregular CC spaces is an interesting open question even in Carnot groups (see [4] for a partial result). However, property $\mathcal{R}$ is satisfied, besides in Euclidean spaces ([13]), in several interesting situations like Heisenberg groups [22], Carnot groups of step 2 [23] and Carnot groups of type $\star$ [35]: in particular, Theorems 1.5, 1.6] and 1.7] below hold is such classes. We conjecture that property $\mathcal{R}$ holds also in all CC spaces of step 2, see [3]. Building on the results of [14], we prove in Section 4 the validity of the weaker property $\mathcal{L} \mathcal{R}$ ("Lipschitz rectifiability", see Definition 3.4) in all Carnot groups satisfying the algebraic property (47) below; in particular, a weaker version of Theorem 1.5 holds in such groups, see Theorem 3.5.

The first result we are able to prove assuming property $\mathcal{R}$ is a refinement of Theorem 1.2 and, roughly speaking, it states that $\mathscr{H}^{Q-1}$-almost all singularities of a $B V_{X}$ function are of jump type.

Theorem 1.5. Let $\left(\mathbb{R}^{n}, X\right)$ be an equiregular $C C$ space satisfying property $\mathcal{R}$, let $\Omega \subseteq \mathbb{R}^{n}$ be an open set and let $u \in B V_{X}\left(\Omega ; \mathbb{R}^{k}\right)$. Then $\mathcal{S}_{u}$ is countably $X$-rectifiable and $\mathscr{H}^{Q-1}\left(\mathcal{S}_{u} \backslash \mathcal{J}_{u}\right)=0$.

Assuming property $\mathcal{R}$, also Theorem 1.3 can be refined as follows.

Theorem 1.6. Under the assumption and notation of Theorem 1.3 , assume that $\left(\mathbb{R}^{n}, X\right)$ satisfies property $\mathcal{R}$. Then

(i) $\mathscr{H}^{Q-1}\left(\Theta_{u} \backslash \mathcal{J}_{u}\right)=0$ and $D_{X}^{j} u=D_{X} u\left\llcorner\Theta_{u}\right.$;

(ii) $D_{X}^{c} u=D_{X} u\left\llcorner\left(S \backslash \Theta_{u}\right)\right.$;

(iii) if $B \subseteq \Omega$ is such that $\mathscr{H}^{Q-1}\left\llcorner B\right.$ is $\sigma$-finite, then $D_{X}^{c} u(B)=D_{X}^{a} u(B)=0$.

Theorem 1.6 is part of Theorem 3.16, We also mention that, assuming property $\mathcal{R}$, one can define a precise representative $u^{\mathfrak{p}}$ of $u$ (see (45)) and prove that the convergence of the mean values $f_{B(p, r)} u d \mathscr{L}^{n}$ to $u^{\mathfrak{p}}(p)$ holds, as $r \rightarrow 0$, for $\mathscr{H}^{Q-1}$-almost every $p$. See Theorem 3.14 .

Eventually, a further natural assumption, property $\mathcal{D}$ ("density", see Definition 3.8), concerning the local behavior of the spherical Hausdorff measure $\mathscr{S}^{Q-1}$ of $C_{X}^{1}$ hypersurfaces, allows to obtain a stronger result about the jump part $D^{j} u$, see Theorem 1.7. Property $\mathcal{D}$ is satisfied in Riemannian manifolds (trivially), Heisenberg groups, Carnot groups of step 2 and Carnot groups of type $\star$, see section 4 , its validity in more general settings is an interesting open problem that will be object of future investigations. Theorem 1.7 follows from the more general 
Theorem 3.10, which deals with a representation of the restriction of $D_{X} u$ to any countably $X$-rectifiable set $R$.

Theorem 1.7. Let $\left(\mathbb{R}^{n}, X\right)$ be an equiregular $C C$ space satisfying properties $\mathcal{R}$ and $\mathcal{D}$; then, there exists a function $\sigma: \mathbb{R}^{n} \times \mathbb{S}^{m-1} \rightarrow(0,+\infty)$ such that, for every open set $\Omega \subseteq \mathbb{R}^{n}$ and every $u \in B V_{X}\left(\Omega ; \mathbb{R}^{k}\right)$, one has

$$
D_{X}^{j} u=\sigma\left(\cdot, \nu_{u}\right)\left(u^{+}-u^{-}\right) \otimes \nu_{u} \mathscr{S}^{Q-1}\left\llcorner\mathcal{J}_{u} .\right.
$$

The paper is structured as follows. In Section 2 we introduce the preliminary material about CC spaces and their nilpotent approximation (Section 2.1), $C_{X}^{1}$ hypersurfaces and $X$ rectifiable sets (Section 2.2), approximate $X$-jumps and $X$-differentiability (Section 2.3) and $B V_{X}$ functions (Section 2.4). Most of the material in Sections 2.2 and 2.3 is original. Section 3 contains the proof of our results, while in Section 4 we discuss some classes of Carnot groups satisfying properties $\mathcal{R}, \mathcal{L} \mathcal{R}$ and/or $\mathcal{D}$. Eventually, we collected in Appendix A some useful result from Geometric Measure Theory in metric spaces and in Appendix B the proofs of some (new but) technical results (Borel regularity, etc.) about the approximate $X$-jump and the approximate $X$-differentiability sets.

Acknowledgements. It is a pleasure to thank V. Magnani, R. Monti, D. Morbidelli and D. Pallara for their interest in this paper and for several stimulating discussions.

\section{PRELiminaries}

2.1. Carnot-Carathéodory spaces and nilpotent approximation. In what follows $\Omega$ will denote an open set in $\mathbb{R}^{n}$ and $X=\left(X_{1}, \ldots, X_{m}\right)$ an $m$-tuple $(m \leq n)$ of smooth and linearly independent vector fields on $\mathbb{R}^{n}$, with $2 \leq m \leq n$. We say that an absolutely continuous curve $\gamma:[0, T] \rightarrow \mathbb{R}^{n}$ is an $X$-subunit path joining $p$ and $q$ if $\gamma(0)=p, \gamma(T)=q$ and there exist $h_{1}, \ldots, h_{m} \in L^{\infty}([0, T] ; \mathbb{R})$ such that $\sum_{j=1}^{m} h_{j}^{2} \leq 1$ and for almost every $t \in[0, T]$ one has

$$
\dot{\gamma}(t)=\sum_{j=1}^{m} h(t) X_{j}(\gamma(t)) .
$$

For every $p, q \in \mathbb{R}^{n}$, we define the quantity

$$
d(p, q):=\inf \{T>0: \exists \text { a } X \text {-subunit path } \gamma \text { joining } p \text { and } q\},
$$

where we agree that $\inf \emptyset=+\infty$.

A sufficient condition that makes $d$ a metric on $\mathbb{R}^{n}$ is the following

Theorem 2.1 (Chow-Rashevsky). Suppose that

$$
\forall p \in \mathbb{R}^{n} \quad \mathcal{L} i e\left\{X_{1}, \ldots, X_{m}\right\}(p)=T_{p} \mathbb{R}^{n} \cong \mathbb{R}^{n},
$$

where $\mathcal{L} i e\left\{X_{1}, \ldots, X_{m}\right\}(p)$ denotes the linear span of all iterated commutators of the vector fields $X_{1}, \ldots, X_{m}$ computed at $p$. Then $d$ is a distance.

We will refer to (2) as Hörmander condition. When (2) holds, the couple $\left(\mathbb{R}^{n}, X\right)$ is said to be a Carnot-Carathéodory space of rank $m$. We denote by $B(p, r)$ the $d$-ball of center $p \in \mathbb{R}^{n}$ and radius $r>0$.

For every $p \in \mathbb{R}^{n}$ and for every $i \in \mathbb{N}$ we denote by $\mathcal{L}^{i}(p)$ the linear span of all the commutators of $X_{1}, \ldots, X_{m}$ up to order $i$ computed at $p$. Notice that $\mathcal{L} i e\left\{X_{1}, \ldots, X_{m}\right\}(p)=\bigcup_{i \in \mathbb{N}} \mathcal{L}^{i}(p)$. We say that $\left(\mathbb{R}^{n}, X\right)$ is equiregular if there exist natural numbers $n_{0}, n_{1}, \ldots, n_{s}$ such that

$$
0=n_{0}<n_{1}<\cdots<n_{s}=n \quad \text { and } \quad \forall p \in \mathbb{R}^{n} \operatorname{dim} \mathcal{L}^{i}(p)=n_{i} .
$$

The natural number $s$ is called step of the Carnot-Carathéodory space. 
In the following theorem we resume some well-known facts about the geometry of equiregular $\mathrm{CC}$ spaces, see e.g. [41, 36]. Recall that a Radon measure $\mu$ on a metric space $(M, d)$ is doubling if there exists $C>0$ such that

$$
\mu(B(x, 2 r)) \leq C \mu(x, r) \quad \text { for every } x \in M \text { and every } r>0 .
$$

Theorem 2.2. Let $\left(\mathbb{R}^{n}, X\right)$ be an equiregular $C C$ space of step s. Then the following facts hold.

(i) For every compact set $K \subseteq \mathbb{R}^{n}$ there exists $M \geq 1$ such that

$$
\frac{1}{M}|p-q| \leq d(p, q) \leq M|p-q|^{\frac{1}{s}} \quad \text { for any } p, q \in K
$$

(ii) The Hausdorff dimension of the metric space $\left(\mathbb{R}^{n}, d\right)$ is $Q:=\sum_{i=1}^{s} i\left(n_{i}-n_{i-1}\right)$.

(iii) The metric measure space $\left(\mathbb{R}^{n}, d, \mathscr{L}^{n}\right)$ is locally Ahlfors $Q$-regular, i.e., for every compact set $K \subseteq \mathbb{R}^{n}$ there exist $R>0$ and $C>1$ such that for every $p \in K$ and for every $r \in(0, R)$

$$
\frac{1}{C} r^{Q} \leq \mathscr{L}^{n}(B(p, r)) \leq C r^{Q} .
$$

In particular, $\left(\mathbb{R}^{n}, d, \mathscr{L}^{n}\right)$ is locally doubling.

As customary, we assume from now on that the metric balls $B(p, r)$ are bounded with respect to the Euclidean metric in $\mathbb{R}^{n}$; this implies that the $\mathrm{CC}$ space $\left(\mathbb{R}^{n}, X\right)$ is geodesic, i.e., that for every $p, q \in \mathbb{R}^{n}$ there exists a $X$-subunit curve realizing the infimum in (1). The existence of length minimizing curves implies that, for every $p \in \mathbb{R}^{n}$ and for every $r>0$, one has $\mathscr{L}^{n}(\partial B(p, r))=0$; see Proposition A.9.

Definition 2.3 (Adapted exponential coordinates). Let $\left(\mathbb{R}^{n}, X\right)$ be an equiregular CC space and let $p \in \mathbb{R}^{n}$ be fixed; choose an open neighborhood $V \subseteq \mathbb{R}^{n}$ of $p$ and smooth vector fields $Y_{1}, \ldots, Y_{n}$ such that

- $Y_{i}=X_{i}$ for any $i=1, \ldots, m$;

- for every $k=1, \ldots, s$ the vector fields $Y_{n_{k-1}+1}, \ldots, Y_{n_{k}}$ are chosen among the $k$-order commutators of $X_{1}, \ldots, X_{m}$;

- for every $q \in V$ and every $k=1, \ldots, s$ the set $\left\{Y_{1}(q), \ldots, Y_{n_{k}}(q)\right\}$ is a basis of $\mathcal{L}^{k}(q)$.

Then there exists a neighborhood $U$ of 0 in $\mathbb{R}^{n}$ for which the map

$$
\begin{aligned}
F: U & \rightarrow \mathbb{R}^{n} \\
x & \mapsto \exp \left(x_{1} Y_{1}+\cdots+x_{n} Y_{n}\right)(p)
\end{aligned}
$$

is well defined. We say that $\left(x_{1}, \ldots, x_{n}\right)$ are adapted exponential coordinates around $p$.

The definition of $F$ depends on the point $p$; when confusion may arise, we underline this dependence by using the notation $F_{p}$ to denote (for any $x \in \mathbb{R}^{n}$ for which it is defined) the map $F_{p}(x):=\exp \left(x_{1} Y_{1}+\cdots+x_{n} Y_{n}\right)(p)$. When needed, we will also write $F(p, x)$ to denote $\exp \left(x_{1} Y_{1}+\cdots+x_{n} Y_{n}\right)(p)$; notice that, for every bounded set $V \subseteq \mathbb{R}^{n}$, one can find an open neighborhood $U$ of 0 in $\mathbb{R}^{n}$ such that $F$ is well defined in $V \times U$.

For every $p \in \mathbb{R}^{n}$ and every $j=1, \ldots, m$ we define

$$
\widetilde{X}_{j}:=d F_{p}^{-1}\left(X_{j} \circ F_{p}\right) .
$$

It is readily seen that if $X$ satisfies the Hörmander condition, then also $\widetilde{X}$ does and we denote by $\widetilde{d}$ the CC distance in (a suitable open subset of) $\mathbb{R}^{n}$ associated with the $m$-tuple of vector fields $\widetilde{X}=\left(\widetilde{X}_{1}, \ldots, \widetilde{X}_{m}\right)$, and by $\widetilde{B}(x, r)$ the metric balls associated with $\widetilde{d}$. Again, when confusion may arise we shall use the notation $\widetilde{B}_{p}(x, r)$ to specify that the metric ball is induced 
by the map $F_{p}$. Since $d F_{p}(0) e_{j}=Y_{j}(p)$, we have $\tilde{X}_{j}(0)=e_{j}$ for every $j=1, \ldots, m$. Moreover it is easy to verify that for every $p \in \mathbb{R}^{n}$ and every sufficiently small $r>0$ one has

$$
d\left(F_{p}\left(x_{1}\right), F_{p}\left(x_{2}\right)\right)=\widetilde{d}\left(x_{1}, x_{2}\right) \quad \forall x_{1}, x_{2} \in \widetilde{B}(0, r) ;
$$

in particular, $F_{p}(\widetilde{B}(x, r))=B\left(F_{p}(x), r\right)$.

Remark 2.4. Let us consider $\mu_{p}:=\left(F_{p}^{-1}\right)_{\#} \mathscr{L}^{n}$, i.e., the measure defined for every Borel set $A$ in $\mathbb{R}^{n}$ by

$$
\mu_{p}(A)=\mathscr{L}^{n}\left(F_{p}(A)\right)=\int_{A}\left|\operatorname{det} \nabla F_{p}\right| d \mathscr{L}^{n}
$$

It is easy to see that, whenever $0<\varepsilon<\left|\operatorname{det} \nabla F_{p}(0)\right|$, there exists an open neighborhood $U$ of 0 such that

$$
\left(\left|\operatorname{det} \nabla F_{p}(0)\right|-\varepsilon\right) \mathscr{L}^{n}\left\llcorner U \leq \mu_{p}\left\llcorner U \leq\left(\left|\operatorname{det} \nabla F_{p}(0)\right|+\varepsilon\right) \mathscr{L}^{n}\llcorner U .\right.\right.
$$

Definition 2.5 (Degree, dilations and pseudo-norm). If $\left(\mathbb{R}^{n}, X\right)$ is an equiregular CC space and $p, Y_{1}, \ldots, Y_{n}$ are as in Definition 2.3, we define the degree $w_{j}$ of the coordinate $j$ by $Y_{j}(p) \in$ $\mathcal{L}^{w_{j}}(p) \backslash \mathcal{L}^{w_{j-1}}(p)$ or, equivalently, by $n_{w_{j-1}}<j \leq n_{w_{j}}$. For every $r>0$, the anisotropic dilation $\delta_{r}: \mathbb{R}^{n} \rightarrow \mathbb{R}^{n}$ is defined by

$$
\delta_{r}(x):=\left(x_{1}, \ldots, r^{w_{i}} x_{i}, \ldots, r^{s} x_{n}\right) .
$$

We say that a function $f: \mathbb{R}^{n} \rightarrow \mathbb{R}$ is $\delta$-homogeneous of degree $w \in \mathbb{N}$ if for every $p \in \mathbb{R}^{n}$ and every $\lambda>0$ one has $f\left(\delta_{\lambda} p\right)=\lambda^{w} f(p)$. We also introduce the pseudo-norm

$$
\|x\|:=\sum_{j=1}^{n}\left|x_{j}\right|^{1 / w_{j}}, \quad x \in \mathbb{R}^{n}
$$

and the pseudo-balls

$$
A(r):=\left\{x \in \mathbb{R}^{n}:\|x\| \leq r\right\}
$$

Clearly, $\delta_{r}(A(1))=A(r)$.

The following result is proved in [41].

Theorem 2.6. Let $K \subseteq \mathbb{R}^{n}$ be a compact set in an equiregular $C C$ space $\left(\mathbb{R}^{n}, X\right)$ and let $U$ be a neighborhood of 0 such that, for every $p \in K$, the map $F_{p}$ is well-defined in $U$. Then there exists $C>1$ such that for every $x \in U$ and every $p \in K$ we have

$$
\frac{1}{C}\|x\| \leq \widetilde{d}_{p}(0, x) \leq C\|x\|
$$

The following theorem is classical, see e.g. [7] or [39]. For an introduction to Carnot groups (also known as stratified groups) see for instance [18, 38, 31, 30].

Theorem 2.7. Let $\left(\mathbb{R}^{n}, X\right)$ be an equiregular $C C$ space and let $p \in \mathbb{R}^{n}$ be fixed; then, there exists a family $\widehat{X}:=\left(\widehat{X}_{1}, \ldots, \widehat{X}_{m}\right)$ of polynomial vector fields in $\mathbb{R}^{n}$ such that

(i) for every $j=1, \ldots, m, \widehat{X}_{j}$ is 1-homogeneous, i.e. $\left(d \delta_{r}\right)\left[\widehat{X}_{j}\right]=r \widehat{X}_{j} \circ \delta_{r}$ for all $r>0$;

(ii) for every $j=1, \ldots, m$ we have $r\left(d \delta_{r^{-1}}\right)\left[\widetilde{X}_{j} \circ \delta_{r}\right] \rightarrow \widehat{X}_{j}$ in $C_{l o c}^{\infty}\left(\mathbb{R}^{n}\right)$;

(iii) the couple $\left(\mathbb{R}^{n}, \widehat{X}\right)$ is associated with a Carnot group structure on $\mathbb{R}^{n}$;

(iv) (399, Remark 2.6]) $\widehat{X}$ can be completed to a basis $\widehat{X}_{1}, \ldots, \widehat{X}_{n}$ of the Lie algebra of the Carnot group in such a way that $x=\exp \left(\sum_{j=1}^{n} x_{j} \widehat{X}_{j}\right)(0)$ for any $x \in \mathbb{R}^{n}$. 
The vector fields $\widehat{X}_{1}, \ldots, \widehat{X}_{m}$ introduced in Theorem 2.7 are known in the literature as the nilpotent approximation of $X_{1}, \ldots, X_{m}$ at the point $p$; we will say that the structure $\left(\mathbb{R}^{n}, \widehat{X}\right)$ is tangent to $\left(\mathbb{R}^{n}, X\right)$ at $p$. We shall denote by $\widehat{d}$ the Carnot-Carathéodory distance associated with $\widehat{X}$ and by $\widehat{B}$ the corresponding balls; recall that $\widehat{d}\left(\delta_{r} x, \delta_{r} y\right)=r \widehat{d}(x, y)$ for any $r>0$ and $x, y \in \mathbb{R}^{n}$. When confusion may arise, we shall use the notation $\widehat{B}_{p}, \widehat{d}_{p}$ to specify the dependence on the point $p$.

By the Carnot group structure there exists $\widehat{C}=\widehat{C}_{p}>0$ such that

$$
\mathscr{L}^{n}\left(\widehat{B}_{p}(x, r)\right)=\widehat{C} r^{Q} \quad \forall x \in \mathbb{R}^{n}, r>0 .
$$

The constant $\widehat{C}$ depends on $p$; however, given a compact set $K \subseteq \mathbb{R}^{n}$, there exists $M>0$ such that $1 / M \leq \widehat{C}_{p} \leq M$ for any $p \in K$. See Remark 2.10 below.

We will need later the following simple result.

Proposition 2.8. Let $\left(\mathbb{R}^{n}, X\right)$ be an equiregular $C C$ space, and let $r>0$. Then for every $p \in \mathbb{R}^{n}$ one has

$$
x \in \widehat{B}_{p}(0, r) \Longleftrightarrow-x \in \widehat{B}_{p}(0, r) .
$$

Proof. By well-known properties of Carnot groups and Theorem 2.7 (iv) we have

$$
-x=\exp \left(-\sum_{j=1}^{n} x_{j} \widehat{X}_{j}\right)(0)=\left[\exp \left(\sum_{j=1}^{n} x_{j} \widehat{X}_{j}\right)(0)\right]^{-1}=x^{-1}
$$

which combined with the left invariance of $\widehat{d}$ with respect to the group operation implies

$$
\widehat{d}(0,-x)=\widehat{d}\left(0, x^{-1}\right)=\widehat{d}\left(x \cdot 0, x \cdot x^{-1}\right)=\widehat{d}(x, 0) .
$$

This concludes the proof.

We recall for future references the following well-known result, for which we refer e.g. to [7, 36].

Theorem 2.9. Let $\left(\mathbb{R}^{n}, X\right)$ be an equiregular $C C$ space and let $p \in \mathbb{R}^{n}$ be fixed; then

$$
\lim _{r \rightarrow 0}\left(\sup \left\{\frac{\left|\widetilde{d}_{p}(x, y)-\widehat{d}_{p}(x, y)\right|}{r}: x, y \in \widetilde{B}_{p}(0, r)\right\}\right)=0 .
$$

In particular, for any $\varepsilon>0$, there exists $R>0$ such that

$$
\widehat{B}_{p}(0,(1-\varepsilon) r) \subseteq \widetilde{B}_{p}(0, r) \subseteq \widehat{B}_{p}(0,(1+\varepsilon) r) \quad \text { for any } r \in(0, R) .
$$

Remark 2.10. Let $K \subseteq \mathbb{R}^{n}$ be a compact set; then there exists $M \geq 1$ such that the constant $\widehat{C}=\widehat{C}_{p}$ appearing in (8) satisfies

$$
\frac{1}{M} \leq \widehat{C}_{p} \leq M \quad \forall p \in K
$$

This follows because, by Theorem 2.9 , for any $p \in K$

$$
\begin{aligned}
\widehat{C}_{p} & =\lim _{r \rightarrow 0} \frac{\mathscr{L}^{n}\left(\widehat{B}_{p}(0, r)\right)}{r^{Q}}=\lim _{r \rightarrow 0} \frac{\mathscr{L}^{n}\left(\widetilde{B}_{p}(0, r)\right)}{r^{Q}}=\lim _{r \rightarrow 0} \frac{\mathscr{L}^{n}\left(F_{p}^{-1}(B(p, r))\right)}{r^{Q}} \\
& =\frac{1}{\left|\operatorname{det} \nabla F_{p}(0)\right|} \lim _{r \rightarrow 0} \frac{\mathscr{L}^{n}(B(p, r))}{r^{Q}}
\end{aligned}
$$

and one can conclude by using Theorem 2.2 (iii) and the smoothness of $F(p, x)$. 
2.2. Hypersurfaces of class $C_{X}^{1}$. This section is devoted to the study of hypersurfaces with intrinsic $C^{1}$ regularity; we work in a fixed equiregular $\mathrm{CC}$ space $\left(\mathbb{R}^{n}, X\right)$. As customary, given an open set $\Omega \subseteq \mathbb{R}^{n}$ we denote by $C_{X}^{1}(\Omega)$ the space of continuous functions $f: \Omega \rightarrow \mathbb{R}$ such that the derivatives $X_{1} f, \ldots, X_{m} f$ are represented, in the sense of distributions, by continuous functions.

Definition 2.11 (Hypersurface of class $C_{X}^{1}$ ). We say that $S \subseteq \mathbb{R}^{n}$ is a $C_{X}^{1}$ hypersurface if for every $p \in S$ there exist $R>0$ and $f \in C_{X}^{1}(B(p, R))$ such that the following facts hold

(i) $S \cap B(p, R)=\{q \in B(p, R): f(q)=0\}$;

(ii) $X f \neq 0$ on $B(p, R)$.

In this case, for every $p$ in $S$ we define the horizontal normal $\nu_{S}(p) \in \mathbb{S}^{m-1}$ to $S$ at $p$ letting

$$
\nu_{S}(p):=\frac{X f(p)}{|X f(p)|} .
$$

The horizontal normal is well-defined up to a sign and, in particular, it does not depend on the choice of $f$ : this is a consequence, for instance, of Corollary 2.14, below.

We will also use the notion of intrinsic Lipschitz regularity for hypersurfaces introduced in [46]. In the next definition, the Lipschitz continuity of $f$ is understood with respect to the CC distance; recall that $f: \Omega \rightarrow \mathbb{R}$ is locally Lipschitz on an open set $\Omega \subseteq \mathbb{R}^{n}$ if and only if it is continuous and its distributional derivatives $X_{1} f, \ldots, X_{m} f$ belong to $L_{l o c}^{\infty}(\Omega)$; see [21, 25].

Definition 2.12 ( $X$-Lipschitz hypersurface). We say that $S \subseteq \mathbb{R}^{n}$ is an $X$-Lipschitz hypersurface if for every $p \in S$ there exist $R>0$ and a Lipschitz map $f: B(p, R) \rightarrow \mathbb{R}$ such that

(i) $B(p, R) \cap S=\{q \in B(p, R): f(q)=0\}$;

(ii) there exist $C>0$ and $1 \leq j \leq m$ such that $X_{j} f \geq C \mathscr{L}^{n}$-a.e. on $B(p, R)$.

Hypersurfaces with $X$-Lipschitz or $C_{X}^{1}$ regularity have locally finite $(Q-1)$-dimensional Hausdorff measure, see [46].

Given $\nu \in \mathbb{R}^{m}$ we define $\widetilde{L}_{\nu}: \mathbb{R}^{n} \rightarrow \mathbb{R}$ letting

$$
\widetilde{L}_{\nu}(x):=\sum_{i=1}^{m} \nu_{i} x_{i}
$$

This notation will be extensively used throughout the paper. The following proposition shows that the maps $\widetilde{L}_{\nu}$ provide a sort of first-order "linear" approximation for $C_{X}^{1}$ functions.

Proposition 2.13. Let $p \in \mathbb{R}^{n}, R>0$ and $f \in C_{X}^{1}(B(p, R))$ be fixed; then

$$
\lim _{r \rightarrow 0}\left(\sup \left\{\frac{\left|f\left(F_{p}(x)\right)-f(p)-\widetilde{L}_{X f(p)}(x)\right|}{r}: x \in \widetilde{B}(0, r)\right\}\right)=0 .
$$

Proof. It is not restrictive to assume that $f(p)=0$. Let $r \leq R$ and take $x \in \widetilde{B}(0, r)$. Set $d:=\widetilde{d}(x, 0)$ and take a geodesic $\gamma \in \operatorname{Lip}\left([0, d] ; \mathbb{R}^{n}\right)$ such that $\gamma(0)=0, \gamma(d)=x$ and there exists $h:[0, d] \rightarrow \mathbb{R}^{m}$ such that for $\mathscr{L}^{1}$-a.e. $t \in[0, d]$ we have

$$
|h(t)|=1 \quad \text { and } \quad \dot{\gamma}(t)=\sum_{j=1}^{m} h_{j}(t) \widetilde{X}_{j}(\gamma(t)) .
$$


Notice that $\widetilde{X}_{j}(0)=e_{j}$, hence there exists $C>0$ such that $\left|\tilde{X}_{j}(y)-e_{j}\right| \leq C r$ for every $y \in \widetilde{B}(0, r)$ and every $j=1, \ldots, m$. Therefore, for every $k=1, \ldots, m$

$$
\begin{aligned}
\left|x_{k}-\int_{0}^{d} h_{k}(t) d t\right| & =\left|\left(\int_{0}^{d} \dot{\gamma}(t) d t\right)_{k}-\int_{0}^{d} h_{k}(t) d t\right| \\
& =\left|\sum_{j=1}^{m} \int_{0}^{d} h_{j}(t)\left(\widetilde{X}_{j}(\gamma(t))\right)_{k} d t-\sum_{j=1}^{m} \int_{0}^{d} h_{j}(t)\left(e_{j}\right)_{k} d t\right| \\
& =\left|\sum_{j=1}^{m} \int_{0}^{d} h_{j}(t)\left(\tilde{X}_{j}(\gamma(t))-e_{j}\right)_{k} d t\right| \leq m C r d \leq m C r^{2}
\end{aligned}
$$

Hence, if for every $x \in \widetilde{B}(0, r)$ we set $d:=\widetilde{d}(x, 0)$ and we denote by $h$ a control associated with the geodesic $\gamma$ joining 0 and $x$, we have

$$
\lim _{r \rightarrow 0}\left(\sup \left\{\frac{1}{r}\left|x_{k}-\int_{0}^{d} h_{k}(t) d t\right|: x \in \widetilde{B}(0, r), k=1, \ldots, m\right\}\right)=0 .
$$

Notice also that for every $x \in \widetilde{B}(0, r)$

$$
\begin{aligned}
f\left(F_{p}(x)\right) & =f\left(F_{p}(x)\right)-f\left(F_{p}(0)\right) \\
& =f\left(F_{p}(\gamma(d))\right)-f\left(F_{p}(\gamma(0))\right)=\int_{0}^{d} \sum_{j=1}^{m} X_{j} f\left(F_{p}(\gamma(t))\right) h_{j}(t) d t .
\end{aligned}
$$

Let $\varepsilon>0$ be fixed. By (11) and the continuity of $X f$ we can choose $r_{0} \in(0, R)$ such that

$$
\begin{array}{ll}
\sup \left\{\frac{1}{r}\left|\left(x_{1}, \ldots, x_{m}\right)-\int_{0}^{d} h(t) d t\right|: x \in \widetilde{B}(0, r)\right\}<\frac{\varepsilon}{2|X f(p)|} & \forall r \in\left(0, r_{0}\right) \\
\left|X f\left(F_{p}(x)\right)-X f(p)\right|<\frac{\varepsilon}{2} & \forall x \in \widetilde{B}\left(0, r_{0}\right) .
\end{array}
$$

For any $r \in\left(0, r_{0}\right)$ and $x \in \widetilde{B}(0, r)$ we have

$$
\begin{aligned}
& \left|f\left(F_{p}(x)\right)-\widetilde{L}_{X f(p)}(x)\right| \\
= & \left|\int_{0}^{d}\left\langle h(t), X f\left(F_{p}(\gamma(t))\right)\right\rangle d t-\sum_{j=1}^{m} X_{j} f(p) x_{j}\right| \\
\leq & \int_{0}^{d}|h(t)|\left|X f\left(F_{p}(\gamma(t))\right)-X f(p)\right| d t+|X f(p)|\left|\left(x_{1}, \ldots, x_{m}\right)-\int_{0}^{d} h(t) d t\right| \\
< & d \frac{\varepsilon}{2}+|X f(p)|\left|\left(x_{1}, \ldots, x_{m}\right)-\int_{0}^{d} h(t) d t\right| .
\end{aligned}
$$

The result follows dividing both sides by $r$ and taking into account that $d \leq r$.

An immediate consequence of Proposition 2.13 is Corollary 2.14, where we start using the following very convenient notation: given $t \in \mathbb{R}$ and a function $f: I \rightarrow \mathbb{R}$ defined on some set $I$, we denote by $\{f>t\},\{f=t\}$, etc. the sets $\{x \in I: f(x)>t\},\{x \in I: f(x)=t\}$, etc. This notation will be extensively used in the paper.

Corollary 2.14. Let $p \in \mathbb{R}^{n}$ and $f \in C_{X}^{1}(B(p, R))$ for some $R>0$; suppose that $f(p)=0$, $X f \neq 0$ in $B(p, R)$ and consider the $C_{X}^{1}$ hypersurface $S:=\{q \in B(p, R): f(q)=0\}$. Then, for every $\varepsilon>0$, there exists $r_{0}>0$ such that, for every $r \in\left(0, r_{0}\right)$

$$
F_{p}^{-1}(S) \cap \widetilde{B}(0, r) \subseteq\left\{x \in \widetilde{B}(0, r):-\varepsilon r \leq \widetilde{L}_{X f(p)}(x) \leq \varepsilon r\right\} .
$$


Moreover

$$
\lim _{r \rightarrow 0} \frac{\mathscr{L}^{n}\left(\left\{x \in \widetilde{B}(0, r): f\left(F_{p}(x)\right) \widetilde{L}_{X f(p)}(x)<0\right\}\right)}{r^{Q}}=0 .
$$

Proof. Fix $\varepsilon>0$ and apply Proposition 2.13 to get $r_{0}>0$ such that for every $0<r<r_{0}$ and for every $x \in \widetilde{B}(0, r)$ we have $\left|f\left(F_{p}(x)\right)-\widetilde{L}_{X f(p)}(x)\right| \leq \varepsilon r$. Then, if we take $x \in \widetilde{B}(0, r) \cap\left\{\widetilde{L}_{X f(p)} \geq\right.$ $2 \varepsilon r\}$, we also get

$$
f\left(F_{p}(x)\right) \geq \varepsilon r .
$$

Reasoning in the same way with the set $\left\{\widetilde{L}_{X f(p)} \leq-2 \varepsilon r\right\}$ we readily get (12). The previous argument shows that for any $\varepsilon>0$ there exists $r_{0}>0$ such that for any $r \in\left(0, r_{0}\right)$ we have

$$
\widetilde{B}(0, r) \cap\left\{\left(f \circ F_{p}\right) \widetilde{L}_{X f(p)} \leq 0\right\} \subseteq \widetilde{B}(0, r) \cap\left\{-\varepsilon r \leq \widetilde{L}_{X f(p)} \leq \varepsilon r\right\} .
$$

The proof of (13) follows by noticing that, by Theorem 2.6

$$
\mathscr{L}^{n}\left(\widetilde{B}(0, r) \cap\left\{-\varepsilon r \leq \widetilde{L}_{X f(p)} \leq \varepsilon r\right\}\right) \leq C \varepsilon r^{Q},
$$

for a suitable constant $C$ independent of $r$.

We point out for future references the following observation.

Remark 2.15. Let $\left(\mathbb{R}^{n}, X\right)$ be an equiregular CC space, $p \in \mathbb{R}^{n}, R>0$ and suppose that $f_{1}, f_{2} \in C_{X}^{1}(B(p, R))$ are such that $f_{1}(p)=f_{2}(p)=0$ and $X f_{1}(p)=X f_{2}(p)$; then one has

$$
\lim _{r \rightarrow 0} \frac{1}{r^{Q}} \mathscr{L}^{n}\left(B(p, r) \cap\left\{f_{1} f_{2} \leq 0\right\}\right)=0 .
$$

Indeed, taking into account (12) we observe that

$$
\lim _{r \rightarrow 0} \frac{1}{r^{Q}} \mathscr{L}^{n}\left(\left\{\xi \in B(p, r): f_{1}(\xi) f_{2}(\xi)=0\right\}\right)=0 .
$$

On the other hand, since $\widetilde{L}_{X f_{1}(p)}=\widetilde{L}_{X f_{2}(p)}$ the set $B(p, r) \cap\left\{f_{1} f_{2}<0\right\}$ is contained in

$$
B(p, r) \cap\left(\left\{f_{1} f_{2}<0 \text { and } \widetilde{L}_{X f_{1}(p)} \circ F_{p}^{-1}>0\right\} \cup\left\{f_{1} f_{2}<0 \text { and } \widetilde{L}_{X f_{1}(p)} \circ F_{p}^{-1} \leq 0\right\}\right),
$$

that combined with (13) completes the proof.

We can now introduce the notion of intrinsic rectifiability in equiregular CC spaces. We denote by $\mathscr{H}^{k}$ and $\mathscr{S}^{k}$, respectively, the $k$-dimensional Hausdorff and spherical Hausdorff measures in $\left(\mathbb{R}^{n}, d\right)$, see e.g. Definition A.3.

Definition 2.16 ( $X$-rectifiability). Let $\left(\mathbb{R}^{n}, X\right)$ be an equiregular CC space of homogeneous dimension $Q \in \mathbb{N}$ and let $R \subseteq \mathbb{R}^{n}$. We say that $R$ is countably $X$-rectifiable (respectively, countably $X$-Lipschitz rectifiable) if there exists a family $\left\{S_{h}: h \in \mathbb{N}\right\}$ of $C_{X}^{1}$ hypersurfaces (resp., $X$-Lipschitz hypersurfaces) such that

$$
\mathscr{H}^{Q-1}\left(R \backslash \bigcup_{h=0}^{\infty} S_{h}\right)=0 .
$$

Moreover we say that $R$ is $X$-rectifiable (resp., $X$-Lipschitz rectifiable) if $R$ is countably $X$ rectifiable (resp., countably $X$-Lipschitz rectifiable) and $\mathscr{H}^{Q-1}(R)<+\infty$.

Definition 2.17 (Horizontal normal). Let $R \subseteq \mathbb{R}^{n}$ be countably $X$-rectifiable and let $\left(S_{h}\right)$ be $C_{X}^{1}$ hypersurfaces such that (14) holds. Then the horizontal normal $\nu_{R}: R \rightarrow \mathbb{S}^{m-1}$ to $R$ is defined by

$$
\nu_{R}(p):=\nu_{S_{h}}(p) \quad \text { if } p \in R \cap S_{h} \backslash \bigcup_{k<h} S_{k}
$$


The horizontal normal $\nu_{R}$ is well-defined, up to a sign, $\mathscr{H}^{Q-1}$-almost everywhere on $R$ : this is a standard consequence of the following result.

Proposition 2.18. Let $\left(\mathbb{R}^{n}, X\right)$ be an equiregular $C C$ space and let $S_{1}, S_{2} \subseteq \mathbb{R}^{n}$ be two hypersurfaces of class $C_{X}^{1}$. Then the set

$$
E:=\left\{p \in S_{1} \cap S_{2}: \nu_{S_{1}}(p) \notin\left\{ \pm \nu_{S_{2}}(p)\right\}\right\}
$$

is $\mathscr{H}^{Q-1}$-negligible.

Proof. By a localization argument we can suppose without loss of generality that $S_{1}$ is bounded in $\mathbb{R}^{n}$ and that $\mathscr{H}^{Q-1}(E) \leq \mathscr{H}^{Q-1}\left(S_{1}\right)<+\infty$. For every $\delta>0$ define

$$
E_{\delta}:=\left\{p \in E:\left|\left\langle\nu_{S_{1}}(p), \nu_{S_{2}}(p)\right\rangle\right| \leq 1-\delta\right\} .
$$

Then we have $E=\bigcup\left\{E_{\delta}: \delta \in(0,+\infty) \cap \mathbb{Q}\right\}$.

Fix $\varepsilon \in(0,1 / 4)$ and define for every $R>0$ the set $E_{\delta, R}$ of all the points $p$ of $E_{\delta}$ such that the following three properties hold for every $r \leq 2 R$

(a) if $C>0$ is the constant appearing in Theorem 2.6, for every $x \in A(C r)$ we have $\widehat{B}_{p}(x, \varepsilon r) \subseteq \widetilde{B}_{p}(x, 2 \varepsilon r)$

(b) for $i=1,2$ we have $F_{p}^{-1}\left(S_{i} \cap B(p, 2 r)\right) \subseteq\left\{\left|\widetilde{L}_{\nu_{S_{i}}(p)}\right|<\varepsilon r\right\}$;

(c) $\operatorname{diam} B(p, r)=\operatorname{diam} \widetilde{B}_{p}(0, r) \geq r$.

By Theorems 2.9 and 2.14 and the fact that diam $\widehat{B}_{p}(0, r)=2 r$ we deduce that $E_{\delta, R} \nearrow E_{\delta}$ as $R \rightarrow 0$. that

Fix now $\eta \in\left(0, \frac{R}{2}\right)$. Then there exist a sequence $\left(q_{h}\right)$ in $\mathbb{R}^{n}$ and a sequence $\left(r_{h}\right)$ in $(0, \eta)$ such

$$
\begin{gathered}
E_{\delta, R} \subseteq \bigcup_{h=0}^{\infty} B\left(q_{h}, r_{h}\right) \quad \text { and } \\
\sum_{h=0}^{\infty}\left(r_{h}\right)^{Q-1} \leq \sum_{h=0}^{\infty}\left(\operatorname{diam} B\left(q_{h}, r_{h}\right)\right)^{Q-1} \leq \mathscr{S}_{\eta}^{Q-1}\left(E_{\delta, R}\right)+1 .
\end{gathered}
$$

We can suppose without loss of generality that for every $h \in \mathbb{N}$ there exists $p_{h} \in B\left(q_{h}, r_{h}\right) \cap E_{\delta, R}$. Therefore for every $h \in \mathbb{N}$ one has $B\left(q_{h}, r_{h}\right) \subseteq B\left(p_{h}, 2 r_{h}\right)$ and consequently

$$
E_{\delta, R} \subseteq \bigcup_{h=0}^{\infty} B\left(p_{h}, 2 r_{h}\right)
$$

Taking into account Theorem [2.6, we can find $C>0$ such that for every $h \in \mathbb{N}$ one has

$$
F_{p_{h}}^{-1}\left(E_{\delta, R} \cap B\left(p_{h}, 2 r_{h}\right)\right) \subseteq A_{h}
$$

where

$$
A_{h}:=\left\{x \in \mathbb{R}^{n}:\|x\| \leq C r_{h} \text { and }\left|\widetilde{L}_{\nu_{S_{i}}\left(p_{h}\right)}(x)\right| \leq \varepsilon r_{h}, \text { for } i=1,2\right\}
$$

We prove now that $\mathscr{L}^{n}\left(A_{h}\right) \leq C_{\delta} \varepsilon^{2} r_{h}^{Q}$ for some $C_{\delta}>0$ depending on $\delta$. In fact, since $\left|\left\langle\nu_{S_{1}}\left(p_{h}\right), \nu_{S_{2}}\left(p_{h}\right)\right\rangle\right| \leq 1-\delta$, we have (up to an orthogonal change of coordinates)

$$
\left\{x \in \mathbb{R}^{n}:\left|\widetilde{L}_{\nu_{S_{i}}\left(p_{h}\right)}(x)\right|<\varepsilon r_{h} \text { for } i=1,2\right\} \subseteq Q^{2}\left(0, C_{\delta} \varepsilon r_{h}\right) \times \mathbb{R}^{n-2},
$$

\footnotetext{
${ }^{1}$ This is an easy consequence of the fact that the curve $t \mapsto \exp \left(t \widehat{X}_{1}\right)$ is globally length minimizing. This fact is well-known to experts, even though the only reference we are aware of is [45, Proposizione 7.4].
} 
where the notation $Q^{2}(z, s)$ denotes a 2-dimensional cube of center $z$ and size $s$. Hence

$$
A_{h} \subseteq\left(Q^{2}\left(0, C_{\delta} \varepsilon r_{h}\right) \cap\left\{x \in \mathbb{R}^{m}: \sum_{j=1}^{m}\left|x_{j}\right| \leq C r_{h}\right\}\right) \times\left\{x \in \mathbb{R}^{n-m}: \sum_{j=m+1}^{n}\left|x_{j}\right|^{\frac{1}{d_{j}}} \leq C r_{h}\right\}
$$

and consequently $\mathscr{L}^{n}\left(A_{h}\right) \leq C_{\delta} \varepsilon^{2} r_{h}^{Q}$.

For every $h \in \mathbb{N}$, combining Theorem A.2 and the fact that $A_{h}$ is compact, we can find $N_{h} \in \mathbb{N}$ and a family $\left\{x_{h, j}: j=1, \ldots, N_{h}\right\}$ of points of $A_{h}$ such that $\left\{\widehat{B}_{p_{h}}\left(x_{h, j}, \varepsilon r_{h}\right): j=1, \ldots, N_{h}\right\}$ covers $A_{h}$ and $\left\{\widehat{B}_{p_{h}}\left(x_{h, j}, \varepsilon \frac{r_{h}}{5}\right): j=1, \ldots, N_{h}\right\}$ is pairwise disjoint. Reasoning as above, it is easy to see that

Therefore we can estimate

$$
\mathscr{L}^{n}\left(\left\{x \in \mathbb{R}^{n}: \widehat{d}_{p_{h}}\left(x, A_{h}\right)<\frac{\varepsilon r_{h}}{5}\right\}\right) \leq \widetilde{C}_{\delta} \varepsilon^{2} r_{h}^{Q} .
$$

$$
N_{h} \leq \frac{\mathscr{L}^{n}\left(\left\{x \in \mathbb{R}^{n}: \widehat{d}_{p_{h}}\left(x, A_{h}\right)<\frac{\varepsilon r_{h}}{5}\right\}\right)}{\mathscr{L}^{n}\left(\widehat{B}_{p_{h}}\left(x_{h, j}, \frac{\varepsilon r_{h}}{5}\right)\right)} \leq \widehat{C}_{\delta} \varepsilon^{2-Q}
$$

for some $\widehat{C}_{\delta}>0$ that, by Remark 2.10, depends only on $\delta$. By property (a) we have also $\widehat{B}_{p_{h}}\left(x_{h, j}, \varepsilon r_{h}\right) \subseteq \widetilde{B}_{p_{h}}\left(x_{h, j}, 2 \varepsilon r_{h}\right)$, hence the family $\left\{\widetilde{B}_{p_{h}}\left(x_{h, j}, 2 \varepsilon r_{h}\right): j=1, \ldots, N_{h}\right\}$ is a covering of $A_{h}$, that is also a covering of $F_{p_{h}}^{-1}\left(E_{\delta, R} \cap B\left(p_{h}, r_{h}\right)\right)$. Hence the family $\left\{B\left(F_{p_{h}}^{-1}\left(x_{h, j}\right), 2 \varepsilon r_{h}\right)\right.$ : $j \in \mathbb{N}\}$ is a covering of $E_{\delta, R} \cap B\left(p_{h}, 2 r_{h}\right)$ In particular, since $\varepsilon \in(0,1 / 4)$ we have

$$
\begin{aligned}
\mathscr{S}_{\eta}^{Q-1}\left(E_{\delta, R}\right) & \leq \mathscr{S}_{4 \varepsilon \eta}^{Q-1}\left(E_{\delta, R}\right) \leq \sum_{h=0}^{\infty} \mathscr{S}_{4 \varepsilon \eta}^{Q-1}\left(E_{\delta, R} \cap B\left(p_{h}, 2 r_{h}\right)\right) \\
& \leq \sum_{h=0}^{\infty} \sum_{j=1}^{N_{h}}\left(\operatorname{diam} B\left(F_{p_{h}}^{-1}\left(x_{h, j}\right), 2 \varepsilon r_{h}\right)\right)^{Q-1} \leq \sum_{h=0}^{\infty} N_{h}\left(4 \varepsilon r_{h}\right)^{Q-1} \\
& \leq \sum_{h=0}^{\infty} \widehat{C}_{\delta} \varepsilon r_{h}^{Q-1} \leq \widehat{C}_{\delta} \varepsilon\left(\mathscr{S}_{\eta}^{Q-1}\left(E_{\delta, R}\right)+1\right) .
\end{aligned}
$$

Letting $\eta \rightarrow 0$ we get $\mathscr{S}^{Q-1}\left(E_{\delta, R}\right) \leq \widehat{C}_{\delta} \varepsilon\left(\mathscr{S}^{Q-1}\left(E_{\delta, R}\right)+1\right)$, which gives, letting $R \rightarrow 0$

$$
\mathscr{S}^{Q-1}\left(E_{\delta}\right) \leq \widehat{C}_{\delta} \varepsilon\left(\mathscr{S}^{Q-1}\left(E_{\delta}\right)+1\right)
$$

Letting now $\varepsilon \rightarrow 0$ we get, for any $\delta>0$, that $\mathscr{S}^{Q-1}\left(E_{\delta}\right)=0$, i.e., $\mathscr{S}^{Q-1}(E)=0$. This concludes the proof.

2.3. Approximate notions of continuity, $X$-jumps and $X$-differentiability. In this section we introduce the notions of approximate continuity, approximate $X$-jumps and approximate $X$-differentiability; we keep on working in a fixed equiregular CC space $\left(\mathbb{R}^{n}, X\right)$. We use the notation

$$
f_{A} u d \mathscr{L}^{n}:=\frac{1}{\mathscr{L}^{n}(A)} \int_{A} u d \mathscr{L}^{n}
$$

and, in what follows, we denote by $\Omega$ an open subset of $\mathbb{R}^{n}$.

Definition 2.19 (Approximate Limit). Let $u \in L_{l o c}^{1}\left(\Omega ; \mathbb{R}^{k}\right), z \in \mathbb{R}^{k}$ and $p \in \Omega$. We say that $z \in \mathbb{R}^{k}$ is the approximate limit of $u$ at $p$ if

$$
\lim _{r \rightarrow 0} f_{B(p, r)}|u-z| d \mathscr{L}^{n}=0
$$

We denote by $u^{\star}(p)$ the approximate limit of $u$ at $p$ and by $\mathcal{S}_{u}$ the set of points in $\Omega$ where $u$ does not admit an approximate limit. 
If the approximate limit of $u$ at a point $p$ exists, it is also unique. By the generalized Lebesgue's differentiation theorem (see e.g. [28, Section 2.7]), we have $\mathscr{L}^{n}\left(\mathcal{S}_{u}\right)=0$ and $u^{\star}=u$ a.e. on $\Omega$. Moreover it can be easily proved (adapting e.g. [2, Proposition 3.64]) that $\mathcal{S}_{u}$ is a Borel set and that $u^{\star}: \Omega \backslash \mathcal{S}_{u} \rightarrow \mathbb{R}^{k}$ is a Borel map.

Remark 2.20. Let $\Omega, u, z$ and $p$ be as in Definition 2.19, Then $u$ has approximate limit $z$ at $p$ if and only if, working in adapted exponential coordinates $F_{p}$ around $p$, as $r \rightarrow 0$ the functions $u \circ F_{p} \circ \delta_{r}$ converge in $L_{l o c}^{1}\left(\mathbb{R}^{n} ; \mathbb{R}^{k}\right)$ to the constant function $z$. This is an easy exercise left to the reader; alternatively, it is enough to follow the proof of Proposition 2.26 below with $a=b=z$.

Definition 2.21 (Essential boundary). Given a measurable set $E \subseteq \mathbb{R}^{n}$ and $t \in[0,1]$ we denote by $E^{t}$ the set of points with density $t$ for $E$, i.e., the set of all $p \in \mathbb{R}^{n}$ satisfying

$$
\lim _{r \rightarrow 0} \frac{\mathscr{L}^{n}(E \cap B(p, r))}{\mathscr{L}^{n}(B(p, r))}=t .
$$

The essential boundary of $E$ is $\partial^{*} E:=\mathbb{R}^{n} \backslash\left(E^{0} \cup E^{1}\right)$.

The following proposition is standard; for the reader's convenience we prove it later in Proposition A.1.

Proposition 2.22. Let $u \in L_{l o c}^{1}(\Omega), p \in \Omega \backslash \mathcal{S}_{u}$ and $t \neq u^{\star}(p)$; then $p \notin \partial^{*}\{u>t\}$.

We now introduce the notion of $X$-jump points; this requires a certain amount of work, one of the reasons being that there is no canonical way of separating a CC ball $B(p, r)$ into complementary "half-balls" $B_{\nu}^{+}(p, r), B_{\nu}^{-}(p, r)$. We will use as separating sets an arbitrary hypersurface $S$ of class $C_{X}^{1}$ such that $\nu_{S}(p)=\nu$, and one of the issues (Remark 2.27 below) is proving well-posedness of our definition independently of the choice of $S$.

For any fixed $p \in \mathbb{R}^{n}, \nu \in \mathbb{S}^{m-1}$ and $r>0$ we introduce the notation $B_{\nu}^{+}(p, r)$ and $B_{\nu}^{-}(p, r)$ as follows. Given $R>0$ and $f \in C_{X}^{1}(B(p, R))$ such that $f(p)=0$ and $X f(p) /|X f(p)|=\nu$, we set for $r \in(0, R)$

$$
B_{\nu}^{+}(p, r):=B(p, r) \cap\{f>0\} \quad \text { and } \quad B_{\nu}^{-}(p, r):=B(p, r) \cap\{f<0\} .
$$

These objects are well-defined only if $r$ is small enough. Moreover, there is a clear abuse of notation, since $B_{\nu}^{ \pm}(p, r)$ depend on the choice of $f$. However, this will not effect the validity of our results.

Before introducing the notion of approximate $X$-jumps we state some properties of the "halfballs" $B_{\nu}^{ \pm}(p, r)$. Proposition 2.23 is used in the proof of Theorem 3.14 .

Proposition 2.23. Let $\left(\mathbb{R}^{n}, X\right)$ be an equiregular $C C$ space and let $\Omega \subseteq \mathbb{R}^{n}$ be an open set. Then, for any $p \in \Omega$ and $\nu \in \mathbb{S}^{m-1}$.

$$
\lim _{r \rightarrow 0} \frac{\mathscr{L}^{n}\left(B_{\nu}^{+}(p, r)\right)}{\mathscr{L}^{n}(B(p, r))}=\lim _{r \rightarrow 0} \frac{\mathscr{L}^{n}\left(B_{\nu}^{-}(p, r)\right)}{\mathscr{L}^{n}(B(p, r))}=\frac{1}{2}
$$

Proof. Let $U$ be a neighborhood of $p$ and let $f \in C_{X}^{1}(U)$ be such that $f(p)=0$ and $X f(p)=\nu$. Choose $\varepsilon \in(0,1)$. By Proposition 2.13 and Theorem 2.9 we can suppose without loss of generality that for every small enough $r$ one has $F_{p}(\widetilde{B}(0, r))=B(p, r)$ and

$$
F_{p}^{-1}\left(B_{\nu}^{+}(p, r)\right)=\widetilde{B}(0, r) \cap\left\{f \circ F_{p}>0\right\} \subseteq \widehat{B}(0,(1+\varepsilon) r) \cap\left\{\widetilde{L}_{\nu} \geq-\varepsilon r\right\} .
$$

Analogously

$$
\widehat{B}(0,(1-\varepsilon) r) \cap\left\{\widetilde{L}_{\nu} \geq \varepsilon r\right\} \subseteq \widetilde{B}(0, r) \cap\left\{f \circ F_{p}>0\right\}=F_{p}^{-1}\left(B_{\nu}^{+}(p, r)\right) .
$$

\footnotetext{
${ }^{2}$ One can consider for instance $f=\widetilde{L}_{\nu} \circ F_{p}$.
} 
Applying $\delta_{1 / r}$ to both sides of (16) and evaluating the Lebesgue measure we get

$$
\begin{aligned}
\frac{\mathscr{L}^{n}\left(F_{p}^{-1}\left(B_{\nu}^{+}(p, r)\right)\right)}{r^{Q}} & =\mathscr{L}^{n}\left(\delta_{1 / r}\left(F_{p}^{-1}\left(B_{\nu}^{+}(p, r)\right)\right)\right) \\
& \leq \mathscr{L}^{n}\left(\widehat{B}(0,1+\varepsilon) \cap\left\{\widetilde{L}_{\nu} \geq-\varepsilon\right\}\right) .
\end{aligned}
$$

Taking the $\lim \sup$ as $r \rightarrow 0$ and letting $\varepsilon \rightarrow 0$ we infer

$$
\limsup _{r \rightarrow 0} \frac{\mathscr{L}^{n}\left(F_{p}^{-1}\left(B_{\nu}^{+}(p, r)\right)\right)}{r^{Q}} \leq \mathscr{L}^{n}\left(\widehat{B}(0,1) \cap\left\{\widetilde{L}_{\nu} \geq 0\right\}\right)=\frac{1}{2} \mathscr{L}^{n}(\widehat{B}(0,1)),
$$

where the last equality follows from Proposition 2.8, With the same argument, from (17) we get

$$
\liminf _{r \rightarrow 0} \frac{\mathscr{L}^{n}\left(F_{p}^{-1}\left(B_{\nu}^{+}(p, r)\right)\right)}{r^{Q}} \geq \frac{1}{2} \mathscr{L}^{n}(\widehat{B}(0,1))
$$

hence

$$
\lim _{r \rightarrow 0} \frac{\mathscr{L}^{n}\left(F_{p}^{-1}\left(B_{\nu}^{+}(p, r)\right)\right)}{r^{Q}}=\frac{1}{2} \mathscr{L}^{n}(\widehat{B}(0,1))
$$

By Theorem 2.9

$$
\lim _{r \rightarrow 0} \frac{\mathscr{L}^{n}(\widetilde{B}(0, r))}{r^{Q}}=\lim _{r \rightarrow 0} \mathscr{L}^{n}\left(\delta_{1 / r}(\widetilde{B}(0, r))\right)=\mathscr{L}^{n}(\widehat{B}(0,1)),
$$

and combining (18) and (19) we get

$$
\lim _{r \rightarrow 0} \frac{\mathscr{L}^{n}\left(F_{p}^{-1}\left(B_{\nu}^{+}(p, r)\right)\right)}{\mathscr{L}^{n}(\widetilde{B}(0, r))}=\frac{1}{2} .
$$

If $c:=|\operatorname{det} \nabla F(0)|>0$, using (15) we notice that for every $0<\varepsilon<c$ and every sufficiently small $r>0$ we have

$$
\frac{(c-\varepsilon) \mathscr{L}^{n}\left(F_{p}^{-1}\left(B_{\nu}^{+}(p, r)\right)\right)}{(c+\varepsilon) \mathscr{L}^{n}(\widetilde{B}(0, r))} \leq \frac{\mathscr{L}^{n}\left(B_{\nu}^{+}(p, r)\right)}{\mathscr{L}^{n}(B(p, r))} \leq \frac{(c+\varepsilon) \mathscr{L}^{n}\left(F_{p}^{-1}\left(B_{\nu}^{+}(p, r)\right)\right)}{(c-\varepsilon) \mathscr{L}^{n}(\widetilde{B}(0, r))} .
$$

The result follows passing to the limit as $r \rightarrow 0$, letting $\varepsilon \rightarrow 0$ and, eventually, using a similar argument for $B_{\nu}^{-}$.

We can now introduce the notion of $X$-jump points.

Definition 2.24 (Approximate $X$-jumps). Let $u \in L_{l o c}^{1}\left(\Omega ; \mathbb{R}^{k}\right)$ and $p \in \Omega$. We say that $u$ has an approximate $X$-jump at $p$ if there exist $a, b \in \mathbb{R}^{k}$ with $a \neq b$ and $\nu \in \mathbb{S}^{m-1}$ such that

$$
\lim _{r \rightarrow 0} f_{B_{\nu}^{+}(p, r)}|u-a| d \mathscr{L}^{n}=\lim _{r \rightarrow 0} f_{B_{\nu}^{-}(p, r)}|u-b| d \mathscr{L}^{n}=0 .
$$

In this case we say that $(a, b, \nu)$ is an approximate $X$-jump triple of $u$ at $p$. We shall denote by $\mathcal{J}_{u}$ the set of approximate $X$-jump points of $u$ and by $\left(u^{+}(p), u^{-}(p), \nu_{u}(p)\right)$ the approximate $X$-jump triple for $u$ at $p \in \mathcal{J}_{u}$.

Remark 2.25. Using e.g. Proposition 2.23 one easily proves that $\mathcal{J}_{u} \subseteq \mathcal{S}_{u}$.

Notice that, if $u$ has an approximate $X$-jump at $p$ associated with $(a, b, \nu)$, then it is also associated with the triple $(b, a,-\nu)$. For this reason, it will be sometimes convenient to consider the space of triples endowed with the equivalence relation $(a, b, \nu) \equiv\left(a^{\prime}, b^{\prime}, \nu^{\prime}\right)$ if and only if $(a, b, \nu)=\left(a^{\prime}, b^{\prime}, \nu^{\prime}\right)$ or $(a, b, \nu)=\left(b^{\prime}, a^{\prime},-\nu^{\prime}\right)$. The following Proposition 2.26 shows that the 
$X$-jump triple $\left(u^{+}(p), u^{-}(p), \nu_{u}(p)\right)$ is unique up to equivalence, for the map $\mathbb{R}^{k} \times \mathbb{R}^{k} \times \mathbb{S}^{m-1} \ni$ $(a, b, \nu) \rightarrow w_{a, b, \nu} \in L_{l o c}^{1}\left(\mathbb{R}^{n} ; \mathbb{R}^{k}\right)$ defined by (21) below satisfies

$$
w_{a, b, \nu}=w_{a^{\prime}, b^{\prime}, \nu^{\prime}} \Longleftrightarrow(a, b, \nu) \equiv\left(a^{\prime}, b^{\prime}, \nu^{\prime}\right) .
$$

In the theory of classical $B V$ functions a jump point can be detected, via a blow-up procedure, in terms of $L_{l o c}^{1}$-convergence to a function taking two different values on complementary halfspaces; this is the content of the next statement, which also gives an equivalent definition of approximate $X$-jump points.

Proposition 2.26. Let $\left(\mathbb{R}^{n}, X\right)$ be an equiregular $C C$ space, $\Omega$ an open set, $u \in L_{\text {loc }}^{1}\left(\Omega ; \mathbb{R}^{k}\right)$, $p \in \Omega$ and let $a, b \in \mathbb{R}^{k}$ with $a \neq b$ and $\nu \in \mathbb{S}^{m-1}$ be fixed. Then the following statements are equivalent:

(i) $p \in \mathcal{J}_{u}$ and $\left(u^{+}(p), u^{-}(p), \nu_{u}(p)\right) \equiv(a, b, \nu)$;

(ii) working in adapted exponential coordinates $F_{p}$ around $p$, as $r \rightarrow 0$ the functions $\widetilde{u}_{r}:=$ $u \circ F_{p} \circ \delta_{r}$ converge in $L_{l o c}^{1}\left(\mathbb{R}^{n} ; \mathbb{R}^{k}\right)$ to

$$
w_{a, b, \nu}(y):= \begin{cases}a & \text { if } \widetilde{L}_{\nu}(y)>0 \\ b & \text { if } \widetilde{L}_{\nu}(y)<0\end{cases}
$$

Proof. We can assume without loss of generality that $k=1$.

We prove the implication (i) $\Rightarrow\left(\right.$ ii); we can assume that $\left(u^{+}(p), u^{-}(p), \nu_{u}(p)\right)=(a, b, \nu)$ and, writing $w:=w_{a, b, \nu}$, we prove that for any fixed $R>0$ one has

$$
\lim _{r \rightarrow 0} \int_{\widehat{B}(0, R)}\left|u \circ F_{p} \circ \delta_{r}-w\right| d \mathscr{L}^{n}=0 .
$$

By a change of variables, this is equivalent to proving that

$$
\lim _{r \rightarrow 0} \frac{1}{r^{Q}} \int_{\widehat{B}(0, r)}\left|u \circ F_{p}-w\right| d \mathscr{L}^{n}=0 .
$$

Let $f$ be the real function of class $C_{X}^{1}$ defined on a neighborhood of $p$ used to define, as in (15), the half-balls $B_{\nu}^{ \pm}(p, r)$ appearing in (20); we set for brevity

$$
\begin{array}{lll}
\widehat{B}_{\nu}^{+}(0, r):=\widehat{B}(0, r) \cap\left\{\widetilde{L}_{\nu}>0\right\}, & \widehat{B}_{\nu}^{-}(0, r):=\widehat{B}(0, r) \cap\left\{\widetilde{L}_{\nu}<0\right\} \\
\widetilde{B}_{\nu}^{+}(0, r):=\widetilde{B}(0, r) \cap\left\{f \circ F_{p}>0\right\}, & & \widetilde{B}_{\nu}^{-}(0, r):=\widetilde{B}(0, r) \cap\left\{f \circ F_{p}<0\right\} .
\end{array}
$$

By Theorem 2.9 there exists an increasing function $\omega:(0,+\infty) \rightarrow(0,+\infty)$ such that

$$
\lim _{r \rightarrow 0} \frac{\omega(r)}{r}=0 \quad \text { and } \quad \widehat{B}(0, r) \subseteq \widetilde{B}(0, r+\omega(r))
$$

for any sufficiently small $r$. Therefore

$$
\begin{aligned}
& \frac{1}{r^{Q}} \int_{\widehat{B}(0, r)}\left|u \circ F_{p}-w\right| d \mathscr{L}^{n} \\
= & \frac{1}{r^{Q}}\left(\int_{\widehat{B}_{\nu}^{+}(0, r)}\left|u \circ F_{p}-a\right| d \mathscr{L}^{n}+\int_{\widehat{B}_{\nu}^{-}(0, r)}\left|u \circ F_{p}-b\right| d \mathscr{L}^{n}\right) \\
\leq & \frac{1}{r^{Q}}\left(\int_{\widetilde{B}_{\nu}^{+}(0, r+\omega(r))}\left|u \circ F_{p}-a\right| d \mathscr{L}^{n}+\int_{\widehat{B}_{\nu}^{+}(0, r) \backslash \widetilde{B}_{\nu}^{+}(0, r+\omega(r))}\left(\left|u \circ F_{p}-b\right|+|a-b|\right) d \mathscr{L}^{n}\right. \\
& \left.\quad+\int_{\widetilde{B}_{\nu}^{-}(0, r+\omega(r))}\left|u \circ F_{p}-b\right| d \mathscr{L}^{n}+\int_{\widehat{B}_{\nu}^{-}(0, r) \backslash \widetilde{B}_{\nu}^{-}(0, r+\omega(r))}\left(\left|u \circ F_{p}-a\right|+|a-b|\right) d \mathscr{L}^{n}\right)
\end{aligned}
$$


and using $\widehat{B}_{\nu}^{ \pm}(0, r) \backslash \widetilde{B}_{\nu}^{ \pm}(0, r+\omega(r)) \subseteq \widetilde{B}(0, r+\omega(r)) \backslash \widetilde{B}_{\nu}^{ \pm}(0, r+\omega(r)) \subseteq \overline{\widetilde{B}_{\nu}^{\mp}(0, r+\omega(r))}$

$$
\begin{aligned}
\leq \frac{1}{r^{Q}}\left(2 \int_{\widetilde{B}_{\nu}^{+}(0, r+\omega(r))}\left|u \circ F_{p}-a\right| d \mathscr{L}^{n}+2 \int_{\widetilde{B}_{\nu}^{-}(0, r+\omega(r))}\left|u \circ F_{p}-b\right| d \mathscr{L}^{n}\right. \\
\left.\quad+|a-b| \mathscr{L}^{n}\left(\widetilde{B}(0, r+\omega(r)) \cap\left\{\left(f \circ F_{p}\right) \widetilde{L}_{\nu} \leq 0\right\}\right)\right)
\end{aligned}
$$

and (22) follows from (20) and Corollary 2.14 taking also Theorem 2.2 into account.

For the converse implication one has to prove that, if (ii) holds and $f$ is a $C_{X}^{1}$ real function on a neighborhood of $p$ such that $f(p)=0$ and $X f(p) /|X f(p)|=\nu$, then (20) holds with $B_{\nu}^{ \pm}(p, r)$ defined (see (15)) in terms of $f$. By Theorem 2.2 and a change of variables, proving (20) amounts to proving that

$$
\lim _{r \rightarrow 0} \frac{1}{r^{Q}} \int_{\widetilde{B}_{\nu}^{+}(0, r)}\left|u \circ F_{p}-a\right| d \mathscr{L}^{n}=\lim _{r \rightarrow 0} \frac{1}{r^{Q}} \int_{\widetilde{B}_{\nu}^{-}(0, r)}\left|u \circ F_{p}-b\right| d \mathscr{L}^{n}=0
$$

and this can be done by a boring adaptation, that we omit, of the previous argument.

Remark 2.27. The proof of Proposition 2.26 implicitly shows that the validity of (20) does not depend on the choice of the function $f$ used in (15) to define $B_{\nu}^{ \pm}(p, r)$.

The proof of the following result is standard and we postpone it to the Appendix B,

Proposition 2.28. Let $\left(\mathbb{R}^{n}, X\right)$ be an equiregular $C C$ space, $\Omega$ be an open set and let $u \in$ $L_{l o c}^{1}\left(\Omega ; \mathbb{R}^{k}\right)$. Then the following facts hold:

(i) $\mathcal{J}_{u}$ is a Borel set and, up to a choice of a representative for $X$-jump triples, the function

$$
\begin{aligned}
\mathcal{J}_{u} & \rightarrow \mathbb{R}^{k} \times \mathbb{R}^{k} \times \mathbb{S}^{m-1} \\
p & \mapsto\left(u^{+}(p), u^{-}(p), \nu_{u}(p)\right)
\end{aligned}
$$

is Borel;

(ii) for every $f \in \operatorname{Lip}\left(\mathbb{R}^{k} ; \mathbb{R}^{h}\right)$ and $p \in \mathcal{J}_{u}$ we have

$$
p \in \mathcal{J}_{f \circ u} \Longleftrightarrow f\left(u^{+}(p)\right) \neq f\left(u^{-}(p)\right)
$$

and in this case $\left((f \circ u)^{+}(p),(f \circ u)^{-}(p), \nu_{f \circ u}(p)\right) \equiv\left(f\left(u^{+}(p)\right), f\left(u^{-}(p)\right), \nu_{u}(p)\right)$. Otherwise, $p \notin \mathcal{S}_{f \circ u}$ and $(f \circ u)^{\star}(p)=f\left(u^{+}(p)\right)=f\left(u^{-}(p)\right)$.

We now pass to he introduction of approximate $X$-differentiability.

Definition 2.29 (Approximate $X$-differentiability). Let $u \in L_{l o c}^{1}\left(\Omega ; \mathbb{R}^{k}\right)$ and $p \in \Omega \backslash \mathcal{S}_{u}$. We say that $u$ is approximately $X$-differentiable at $p$ if there exist a neighborhood $U$ of $p$ and $f \in C_{X}^{1}\left(U ; \mathbb{R}^{k}\right)$ such that $f(p)=0$ and

$$
\lim _{r \rightarrow 0} f_{B(p, r)} \frac{\left|u-u^{\star}(p)-f\right|}{r} d \mathscr{L}^{n}=0 .
$$

The subset of points of $\Omega$ in which $u$ is approximately $X$-differentiable is denoted by $\mathcal{D}_{u}$.

If $f$ is as in Definition 2.29 we will call $X f(p) \in \mathbb{R}^{k \times m}$ the approximate $X$-gradient of $u$ at $p$. By the following proposition the approximate $X$-gradient of $u$ at $p$ is uniquely determined, and we denote it by $D_{X}^{a p} u(p)$.

Proposition 2.30 (Uniqueness of approximate $X$-gradient). Let ( $\left.\mathbb{R}^{n}, X\right)$ be an equiregular $C C$ space, $\Omega \subseteq \mathbb{R}^{n}$ an open set, $u \in L_{\text {loc }}^{1}\left(\Omega ; \mathbb{R}^{k}\right)$ and $p \in \Omega \backslash \mathcal{S}_{u}$. Let $R>0$ and $f_{1}, f_{2} \in$ $C_{X}^{1}\left(B(p, R) ; \mathbb{R}^{k}\right)$; suppose that formula (23) holds for both $f=f_{1}$ and $f=f_{2}$. Then $p \in \mathcal{D}_{u}$, $f_{1}(p)=f_{2}(p)=0$ and $X f_{1}(p)=X f_{2}(p)$.

Conversely, if $f_{1}(p)=f_{2}(p)=0$ and $X f_{1}(p)=X f_{2}(p)$, then formula (23) holds for $f=f_{1}$ if and only if it holds for $f=f_{2}$. 
Proof. It is not restrictive to assume that $k=1$. Define for $i=1,2$ the functions $L_{i}:=\widetilde{L}_{X f_{i}(p)}$. Suppose first that both $f_{1}, f_{2}$ satisfy (23). Fix $\varepsilon>0$ and by Proposition 2.13 choose $r>0$ such that for every $\varrho \in(0, r)$

$$
\frac{\left|f_{i} \circ F_{p}-L_{i}\right|}{\varrho}<\frac{\varepsilon}{2} \quad \text { on } \widetilde{B}(0, \varrho) .
$$

Then for such values of $\varrho$ we have

$$
\begin{aligned}
f_{\widetilde{B}(0, \varrho)} \frac{\left|L_{1}-L_{2}\right|}{\varrho} d \mathscr{L}^{n} & \leq f_{\widetilde{B}(0, \varrho)} \frac{\left|f_{1} \circ F_{p}-f_{2} \circ F_{p}\right|}{\varrho} d \mathscr{L}^{n}+\varepsilon \\
& \leq C f_{B(p, \varrho)} \frac{\left|f_{1}-f_{2}\right|}{\varrho} d \mathscr{L}^{n}+\varepsilon \\
& \leq C f_{B(p, \varrho)} \frac{\left|u-u^{\star}(p)-f_{1}\right|+\left|u-u^{\star}(p)-f_{2}\right|}{\varrho} d \mathscr{L}^{n}+\varepsilon
\end{aligned}
$$

It follows that

$$
\lim _{\varrho \rightarrow 0} f_{\widetilde{B}(0, \varrho)} \frac{\left|L_{1}-L_{2}\right|}{\varrho} d \mathscr{L}^{n}=0 .
$$

If $X f_{1}(p) \neq X f_{2}(p)$, by Theorem 2.6 one would get, for some $C_{1}>0$

$$
\begin{aligned}
f_{\widetilde{B}(0, \varrho)}\left|L_{1}-L_{2}\right| d \mathscr{L}^{n} & =\frac{1}{\mathscr{L}^{n}(\widetilde{B}(0, \varrho))} \int_{\widetilde{B}(0, \varrho)}\left|L_{1}-L_{2}\right| d \mathscr{L}^{n} \\
& \geq \frac{1}{\mathscr{L}^{n}\left(A\left(C_{1} \varrho\right)\right)} \int_{A\left(\varrho / C_{1}\right)}\left|L_{1}-L_{2}\right| d \mathscr{L}^{n}=C \frac{\varrho^{Q+1}}{\varrho^{Q}}=C \varrho,
\end{aligned}
$$

a contradiction. This proves the first part of the statement.

Suppose now that $X f_{1}(p)=X f_{2}(p)$ and that $f_{1}$ satisfies (23). Then we have $L_{1}=L_{2}$ and

$$
\begin{aligned}
& f_{B(p, \varrho)} \frac{\left|u-u^{\star}(p)-f_{2}\right|}{\varrho} d \mathscr{L}^{n} \\
\leq & f_{B(p, \varrho)} \frac{\left|f_{1}-L_{1} \circ F_{p}^{-1}\right|+\left|u(y)-u^{\star}(p)-f_{1}\right|+\left|f_{2}-L_{2} \circ F_{p}^{-1}\right|}{\varrho} d \mathscr{L}^{n} .
\end{aligned}
$$

By Proposition 2.13 this completes the proof.

As for $X$-jump points, also approximate $X$-differentiability points can be detected by a blowup procedure.

Proposition 2.31. Let $\left(\mathbb{R}^{n}, X\right)$ be an equiregular $C C$ space, $\Omega$ be an open subset of $\mathbb{R}^{n}$, $u \in$ $L_{\text {loc }}^{1}\left(\Omega ; \mathbb{R}^{k}\right)$ and let $p \in \Omega \backslash \mathcal{S}_{u}$. Then $u$ is approximate $X$-differentiable at $p$ if and only if there exists $z=\left(z_{1}, \ldots, z_{k}\right) \in \mathbb{R}^{k \times m}$ such that

$$
\frac{u \circ F_{p} \circ \delta_{r}-u^{\star}(p)}{r} \rightarrow\left(\widetilde{L}_{z_{1}}, \ldots, \widetilde{L}_{z_{k}}\right) \quad \text { in } L_{l o c}^{1}\left(\mathbb{R}^{n} ; \mathbb{R}^{k}\right) \text { as } r \rightarrow 0 .
$$

In this case we have $D_{X}^{a p} u(p)=z$. 
Proof. We assume without loss of generality that $k=1$. Assume first that $p \in \mathcal{D}_{u}$ and let $f$ be as in (23); set $z:=D_{X}^{a p} u(p) \in \mathbb{R}^{m}$. Given $R>0$, by Theorem 2.9 one has for small enough $r$

$$
\begin{aligned}
& \int_{\widehat{B}(0, R)}\left|\frac{u \circ F_{p} \circ \delta_{r}-u^{\star}(p)}{r}-\widetilde{L}_{z}\right| d \mathscr{L}^{n} \\
= & \frac{1}{r^{Q}} \int_{\widehat{B}(0, r R)}\left|\frac{u \circ F_{p}-u^{\star}(p)-\widetilde{L}_{z}}{r}\right| d \mathscr{L}^{n} \leq \frac{1}{r^{Q}} \int_{\widetilde{B}(0,2 r R)}\left|\frac{u \circ F_{p}-u^{\star}(p)-\widetilde{L}_{z}}{r}\right| d \mathscr{L}^{n} \\
\leq & \frac{C}{r^{Q}} \int_{B(p, 2 r R)}\left|\frac{u-u^{\star}(p)-\widetilde{L}_{z} \circ F_{p}^{-1}}{r}\right| d \mathscr{L}^{n} \rightarrow 0 \quad \text { as } r \rightarrow 0,
\end{aligned}
$$

(we used Proposition 2.30), which proves the first part of the statement.

Conversely, for any small enough $r>0$ we have

$$
\begin{aligned}
& f_{B(p, r)}\left|\frac{u-u^{\star}(p)-\widetilde{L}_{z} \circ F_{p}^{-1}}{r}\right| d \mathscr{L}^{n} \leq \frac{C}{r^{Q}} \int_{\widetilde{B}(0, r)}\left|\frac{u \circ F_{p}-u^{\star}(p)-\widetilde{L}_{z}}{r}\right| d \mathscr{L}^{n} \\
\leq & \frac{C}{r^{Q}} \int_{\widehat{B}(0,2 r)}\left|\frac{u \circ F_{p}-u^{\star}(p)-\widetilde{L}_{z}}{r}\right| d \mathscr{L}^{n}=C \int_{\widehat{B}(0,2)}\left|\frac{u \circ F_{p} \circ \delta_{r}-u^{\star}(p)}{r}-\widetilde{L}_{z}\right| d \mathscr{L}^{n},
\end{aligned}
$$

which allows to conclude.

The proofs of the following two results are postponed to Appendix B,

Proposition 2.32 (Properties of approximate differentiability points). Let $\left(\mathbb{R}^{n}, X\right)$ be an equiregular $C C$ space, $\Omega$ be an open set in $\mathbb{R}^{n}$ and let $u \in L_{l o c}^{1}\left(\Omega ; \mathbb{R}^{k}\right)$. Then $\mathcal{D}_{u}$ is a Borel set and the map $D_{X}^{a p} u: \mathcal{D}_{u} \rightarrow \mathbb{R}^{m \times k}$ is a Borel map.

Proposition 2.33 (Locality). Let $\left(\mathbb{R}^{n}, X\right)$ be an equiregular $C C$ space, $\Omega$ an open set in $\mathbb{R}^{n}$ and $u, v \in L_{\text {loc }}^{1}\left(\Omega ; \mathbb{R}^{k}\right)$. Suppose that $p \in \Omega$ is of density 1 for the set $\{q \in \Omega: u(q)=v(q)\}$. Then the following facts hold.

(i) If $p \in \Omega \backslash\left(\mathcal{S}_{u} \cup \mathcal{S}_{v}\right)$, then $u^{\star}(p)=v^{\star}(p)$.

(ii) If $p \in \mathcal{J}_{u} \cap \mathcal{J}_{v}$, then $\left(u^{+}(p), u^{-}(p), \nu_{u}(p)\right) \equiv\left(v^{+}(p), v^{-}(p), \nu_{v}(p)\right)$.

(iii) If $p \in \mathcal{D}_{u} \cap \mathcal{D}_{v}$ then $D_{X}^{a p} u(p)=D_{X}^{a p} v(p)$.

2.4. Functions with bounded $X$-variation. In this section we review the definition and basic properties of $B V_{X}$ functions. We keep on working in a fixed equiregular CC space $\left(\mathbb{R}^{n}, X\right)$, while $\Omega$ denotes a fixed open subset of $\mathbb{R}^{n}$.

Definition 2.34 (Functions with bounded $X$-variation). We say that $u \in L_{l o c}^{1}(\Omega)$ is a function of locally bounded $X$-variation in $\Omega$, and we write $u \in B V_{X, l o c}(\Omega)$, if there exists a $\mathbb{R}^{m}$-valued Radon measure $D_{X} u=\left(D_{X_{1}} u, \ldots, D_{X_{m}} u\right)$ in $\Omega$ such that for every open set $A \Subset \Omega$ and for every $\varphi \in C_{c}^{1}(A)$ we have

$$
\forall i=1, \ldots, m \quad \int_{A} \varphi d\left(D_{X_{i}} u\right)=-\int_{A} u X_{i}^{*} \varphi d \mathscr{L}^{n},
$$

where $X_{i}^{*}$ denotes the formal adjoint of $X_{i}$. If $u \in L^{1}(\Omega)$, we say that $u$ has bounded $X$-variation in $\Omega$, and we write $u \in B V_{X}(\Omega)$, if, moreover, the total variation $\left|D_{X} u\right|$ of $D_{X} u$ is finite on $\Omega$.

As customary, we write $B V_{X}\left(\Omega ; \mathbb{R}^{k}\right):=\left(B V_{X}(\Omega)\right)^{k}$, and similarly for $B V_{X, l o c}\left(\Omega ; \mathbb{R}^{k}\right)$. It can be useful to observe that if $u \in B V_{X}\left(\Omega ; \mathbb{R}^{k}\right)$, the following inequalities hold

$$
\max _{1 \leq i \leq k}\left|D_{X} u^{i}\right|(\Omega) \leq\left|D_{X} u\right|(\Omega) \leq \sum_{i=1}^{k}\left|D_{X} u^{i}\right|(\Omega) .
$$


The following approximation result is proved in [20, 24].

Theorem 2.35. Let $u \in B V_{X}\left(\Omega ; \mathbb{R}^{k}\right)$. Then there exists a sequence $\left(u_{h}\right)$ in $C^{\infty}\left(\Omega ; \mathbb{R}^{k}\right)$ such that

$$
\lim _{h}\left\|u_{h}-u\right\|_{L^{1}\left(\Omega ; \mathbb{R}^{k}\right)}=0 \quad \text { and } \quad \lim _{h}\left|D_{X} u_{h}\right|(\Omega)=\left|D_{X} u\right|(\Omega) .
$$

We now state and prove a simple but useful result.

Proposition 2.36. Let $\Omega, \widetilde{\Omega}$ be two open sets in $\mathbb{R}^{n}$ and let $G: \Omega \rightarrow \widetilde{\Omega}$ be a diffeomorphism. Let also $X_{1}, \ldots, X_{m}$ be vector fields on $\Omega$ and define for every $i=1, \ldots, m$ the vector fields $Y_{i}:=d G\left(X_{i}\right)$ on $\widetilde{\Omega}$. Then

$$
u \in B V_{X, l o c}(\Omega) \Longleftrightarrow v:=u \circ G^{-1} \in B V_{Y, l o c}(\widetilde{\Omega}) .
$$

More precisely, for every open set $U \Subset \Omega$ and setting $V:=G(U)$, one has for every $u \in$ $B V_{X, l o c}(\Omega)$ that

$$
m\left|D_{X} u\right|(U) \leq\left|D_{Y} v\right|(V) \leq M\left|D_{X} u\right|(U)
$$

for $m:=\inf _{U}|\operatorname{det} \nabla G|$ and $M:=\sup _{U}|\operatorname{det} \nabla G|$.

Proof. We claim that, for any open set $U \Subset \Omega$ and any $u \in B V_{X, l o c}(\Omega)$, one has

$$
v:=u \circ G^{-1} \in B V_{Y}(V) \quad \text { and } \quad\left|D_{Y} v\right|(V) \leq M\left|D_{X} u\right|(U) .
$$

This would be enough to conclude: indeed, the claim would imply both the $\Rightarrow$ implication in (26) and the second inequality in (27), while the $\Leftarrow$ implication in (26) and the first inequality in (27) simply follow by replacing $X, U, u, G$ with (respectively) $Y, V, v, G^{-1}$ and noticing that $m=\left(\sup _{V}\left|\operatorname{det} \nabla\left(G^{-1}\right)\right|\right)^{-1}$.

Let us prove the claim. First we assume that $u \in C^{\infty}(U)$, so that also $v$ is smooth on $V$. For every $\varphi \in C_{c}^{1}\left(V ; \mathbb{R}^{m}\right)$ with $|\varphi| \leq 1$, by a change of variable we have that

$$
\int_{V}\langle Y v, \varphi\rangle d \mathscr{L}^{n}=\int_{U}\langle X u,(\varphi \circ G)\rangle|\operatorname{det} \nabla G| d \mathscr{L}^{n}
$$

which gives

$$
\left|D_{Y} v\right|(V) \leq M\left|D_{X} u\right|(U) .
$$

In case $u \in B V_{X}(U)$ is not smooth, consider a sequence $\left(u_{h}\right)$ in $C^{\infty}(U)$ that converges to $u$ in $L^{1}(U)$ and such that

$$
\lim _{h}\left|D_{X} u_{h}\right|(U)=\left|D_{X} u\right|(U) .
$$

Defining $v_{h}:=u_{h} \circ G^{-1}$, we easily get that $v_{h}$ converges to $v$ in $L^{1}(V)$ as $h \rightarrow+\infty$. Therefore

$$
\left|D_{Y} v\right|(V) \leq \liminf _{h}\left|D_{Y} v_{h}\right|(V) \leq M \liminf _{h}\left|D_{X} u_{h}\right|(U)=M\left|D_{X} u\right|(U)
$$

and the proof is accomplished.

Definition 2.37 (Sets with finite $X$-perimeter). A measurable set $E \subseteq \mathbb{R}^{n}$ has locally finite $X$-perimeter (resp., finite $X$-perimeter) in $\Omega$ if $\chi_{E} \in B V_{X, l o c}(\Omega)$ (resp., $\chi_{E} \in B V_{X}(\Omega)$ ). In such a case we define the $X$-perimeter measure $P_{X}^{E}$ of $E$ by $P_{X}^{E}:=\left|D_{X} \chi_{E}\right|$.

It will sometimes be useful to write $P_{X}(E, \cdot)$ instead of $P_{X}^{E}$.

Definition 2.38 (Measure theoretic horizontal normal). If $E$ is a set with locally finite $X$ perimeter, then by Riesz representation theorem there exists a $P_{X}^{E}$-measurable function $\nu_{E}$ : $\mathbb{R}^{n} \rightarrow \mathbb{S}^{m-1}$ such that

$$
D_{X} \chi_{E}=\nu_{E} P_{X}^{E}
$$

We call $\nu_{E}$ the measure theoretic horizontal normal to $E$.

The following result is proved in [1] and it will be of capital importance in the following. 
Theorem 2.39. Let $\left(\mathbb{R}^{n}, X\right)$ be an equiregular $C C$ space of homogeneous dimension $Q$; let $E \subseteq \mathbb{R}^{n}$ be a set with finite $X$-perimeter in an open set $\Omega \subseteq \mathbb{R}^{n}$. Then

$$
P_{X}^{E}\left\llcorner\Omega=\theta \mathscr{H}^{Q-1}\left\llcorner\left(\Omega \cap \partial^{*} E\right)\right.\right.
$$

for a suitable positive function $\theta$ that is locally bounded away from 0 . Moreover

$$
\limsup _{r \rightarrow 0} \frac{P_{X}^{E}(B(p, 2 r))}{P_{X}^{E}(B(p, r))}<\infty \quad \text { for } P_{X}^{E} \text {-a.e. } p \in \Omega \cap \partial^{*} E .
$$

The proofs of the following well-known result can be found, for instance, in [20].

Theorem 2.40 (Coarea Formula for $B V_{X}$ functions). Let $\left(\mathbb{R}^{n}, X\right)$ be a $C C$ space, let $\Omega$ be an open set in $\mathbb{R}^{n}$ and let $u \in B V_{X}(\Omega)$. Then, if we define $E_{s}:=\{p \in \Omega: u(p)>s\}$, we have

$$
\left|D_{X} u\right|(\Omega)=\int_{-\infty}^{+\infty} P_{X}\left(E_{s} ; \Omega\right) d s
$$

The next result is essentially [10, Theorem 1.2]; note, however, that the dimension $Q$ appearing in [10, Theorem 1.2] is slightly different from the homogeneous dimension we are considering. See also [29].

Theorem 2.41. Let $\Omega$ be an open subset of an equiregular $C C$ space $\left(\mathbb{R}^{n}, X\right)$ of homogeneous dimension $Q$ and let $K \subseteq \Omega$ be compact. Then there exist $C>0$ and $R>0$ such that, for every $p \in K, r \in(0, R)$ and $u \in B V_{X, l o c}\left(\Omega ; \mathbb{R}^{k}\right)$, the inequality

$$
\left(f_{B(p, r)}\left|u-u_{p, r}\right|^{\frac{Q}{Q-1}} d \mathscr{L}^{n}\right)^{\frac{Q-1}{Q}} \leq \frac{C}{r^{Q-1}}\left|D_{X} u\right|(B(p, r)),
$$

where $u_{p, r}:=f_{B(p, r)}$ ud $\mathscr{L}^{n}$, holds.

Proof. It is clearly enough to consider the case $k=1$. The proof then easily follows by [27, Theorem 5.1] on taking into account Theorem [2.2, [10, Theorem 1.1], [27, Corollary 9.8 and Theorem 10.3] and Theorem 2.35,

An easy consequence of Theorem 2.41 is the following isoperimetric inequality.

Theorem 2.42 (Isoperimetric inequality in $\mathrm{CC}$ spaces). Let $\left(\mathbb{R}^{n}, X\right)$ be an equiregular $C C$ space and let $K \subseteq \mathbb{R}^{n}$ be a compact set. Then there exist $C>0$ and $R>0$ such that, for every $p \in K, r \in(0, R)$ and every $\mathscr{L}^{n}$-measurable set $E \subseteq \mathbb{R}^{n}$, one has

$$
\min \left\{\mathscr{L}^{n}(E \cap B(p, r)), \mathscr{L}^{n}(B(p, r) \backslash E)\right\}^{\frac{Q-1}{Q}} \leq C P_{X}(E, B(p, r)) .
$$

We conclude this section with some auxiliary results. The first one is proved in [16].

Theorem 2.43. Let $X=\left(X_{1}, \ldots, X_{m}\right)$ and $X^{j}=\left(X_{1}^{j}, \ldots, X_{m}^{j}\right), j \in \mathbb{N}$, be m-tuples of linearly independent smooth vector fields on $\mathbb{R}^{n}$ such that $X$ satisfies the Hörmander condition and its $C C$ balls are bounded in $\mathbb{R}^{n}$; assume that, for every $i=1, \ldots, m, X_{i}^{j} \rightarrow X_{i}$ in $C_{l o c}^{\infty}\left(\mathbb{R}^{n}\right)$ as $j \rightarrow \infty$. Let $u_{j} \in B V_{X^{j}, \text { loc }}\left(\mathbb{R}^{n}\right)$ be a sequence of functions that is locally uniformly bounded in $B V_{X^{j}}$, i.e., such that for any compact set $K \subseteq \mathbb{R}^{n}$ there exists $M>0$ such that

$$
\forall j \in \mathbb{N} \quad\left\|u_{j}\right\|_{L^{1}(K)}+\left|D_{X^{j}} u_{j}\right|(K) \leq M<\infty .
$$

Then, there exist $u \in B V_{X, l o c}\left(\mathbb{R}^{n}\right)$ and a subsequence $\left(u_{j_{h}}\right)$ of $\left(u_{j}\right)$ such that $u_{j_{h}} \rightarrow u$ in $L_{\text {loc }}^{1}\left(\mathbb{R}^{n}\right)$ as $h \rightarrow \infty$. Moreover, for any bounded open set $\Omega \subseteq \mathbb{R}^{n}$ one has

$$
\left|D_{X} u\right|(\Omega) \leq \liminf _{j \rightarrow \infty}\left|D_{X^{j}} u_{j}\right|(\Omega)
$$


The proof of Theorem 2.43 given in [16] implicitly contains also the following result's proof, that we however provide for the sake of completeness.

Proposition 2.44. Let $X=\left(X_{1}, \ldots, X_{m}\right)$ and $X^{j}=\left(X_{1}^{j}, \ldots, X_{m}^{j}\right), j \in \mathbb{N}$, be m-tuples of linearly independent smooth vector fields on $\mathbb{R}^{n}$ such that, for every $i=1, \ldots, m, X_{i}^{j} \rightarrow X_{i}$ in $C_{\text {loc }}^{\infty}\left(\mathbb{R}^{n}\right)$ as $j \rightarrow \infty$. Let $\left(u_{j}\right) \subseteq L_{\text {loc }}^{1}\left(\mathbb{R}^{n}\right)$ be a sequence converging in $L_{\text {loc }}^{1}\left(\mathbb{R}^{n}\right)$ to some $u$; then, for any open bounded set $\Omega \subseteq \mathbb{R}^{n}$ one has

$$
\left|D_{X} u\right|(\Omega) \leq \liminf _{j \rightarrow \infty}\left|D_{X^{j}} u_{j}\right|(\Omega)
$$

Proof. For any $i=1, \ldots, m$ and any $j \in \mathbb{N}$ we write

$$
X_{i}(x)=\sum_{k=1}^{n} a_{i, k}(x) \partial_{k} \quad \text { and } \quad X_{i}^{j}(x)=\sum_{k=1}^{n} a_{i, k}^{j}(x) \partial_{k}
$$

for suitable smooth functions $a_{i, k}, a_{i, k}^{j}$. Then, for any test function $\varphi \in C_{c}^{1}\left(\Omega ; \mathbb{R}^{m}\right)$ we have

$$
\begin{aligned}
\int_{\Omega} u \sum_{i=1}^{m} X_{i}^{*} \varphi_{i} d \mathscr{L}^{n} & =\int_{\Omega} u \sum_{i=1}^{m} \sum_{k=1}^{n} \partial_{k}\left(a_{i, k} \varphi_{i}\right) d \mathscr{L}^{n}=\lim _{j \rightarrow \infty} \int_{\Omega} u_{j} \sum_{i=1}^{m} \sum_{k=1}^{n} \partial_{k}\left(a_{i, k}^{j} \varphi_{i}\right) d \mathscr{L}^{n} \\
& =\lim _{j \rightarrow \infty} \int_{\Omega} u_{j} \sum_{i=1}^{m} X_{i}^{j^{*}} \varphi_{i} d \mathscr{L}^{n} \leq\|\varphi\|_{L^{\infty}(\Omega)} \liminf _{j \rightarrow \infty}\left|D_{X^{j}} u_{j}\right|(\Omega) .
\end{aligned}
$$

The proof is accomplished.

Remark 2.45. Let $X, X^{j}, u_{j}, u$ be as in Proposition 2.44 and assume that $\left|D_{X^{j}} u^{j}\right|$ are locally uniformly bounded in $\mathbb{R}^{n}$, i.e., for any compact set $K \subseteq \mathbb{R}^{n}$ there exists $C_{K}<\infty$ such that $\left|D_{X^{j}} u^{j}\right|(K)<C_{K}$ for all $j$. Then $D_{X^{j}} u^{j}$ weakly* converges to $D_{X} u$ in $\mathbb{R}^{n}$.

Indeed, one can reason as in (29) to show that for any test function $\varphi \in C_{c}^{1}\left(\mathbb{R}^{n}\right)$ and any $i=1, \ldots, m$

$$
\lim _{j \rightarrow \infty} \int \varphi d D_{X_{i}^{j}} u^{j}=\int \varphi d D_{X_{i}} u
$$

and the density of $C_{c}^{1}$ in $C_{c}^{0}$ allows to conclude.

\section{Fine properties of BV functions}

This section is devoted to the proof of our main results.

Lemma 3.1. Let $\left(\mathbb{R}^{n}, X\right)$ be an equiregular $C C$ space, let $\Omega \subseteq \mathbb{R}^{n}$ be open and let $\left(E_{h}\right)$ be a sequence of measurable sets in $\Omega$ such that

$$
\lim _{h} \mathscr{L}^{n}\left(E_{h}\right)=0 \quad \text { and } \quad \lim _{h} P_{X}\left(E_{h} ; \Omega\right)=0 .
$$

Then for every $\alpha \in(0,1)$ we have

$$
\mathscr{H}^{Q-1}\left(\bigcap_{h=1}^{\infty}\left\{p \in \Omega: \limsup _{r \rightarrow 0} \frac{\mathscr{L}^{n}\left(E_{h} \cap B(p, r)\right)}{\mathscr{L}^{n}(B(p, r))} \geq \alpha\right\}\right)=0 .
$$

Proof. Set

$$
E_{h}^{\alpha}:=\left\{q \in \Omega: \limsup _{r \rightarrow 0} \frac{\mathscr{L}^{n}\left(E_{h} \cap B(q, r)\right)}{\mathscr{L}^{n}(B(q, r))} \geq \alpha\right\},
$$

and suppose without loss of generality that $\mathscr{L}^{n}\left(E_{h}\right)>0$ for every $h \in \mathbb{N}$. Let $K \Subset \Omega$. By Theorem 2.2 there exist $C>1$ and $R>0$ such that for every $q \in K$, for every $0<r<2 R$ we have

$$
\frac{1}{C} r^{Q} \leq \mathscr{L}^{n}(B(q, r)) \leq C r^{Q}
$$


For any sufficiently large $h \in \mathbb{N}$ we have

$$
\left(\frac{2 C \mathscr{L}^{n}\left(E_{h}\right)}{\alpha}\right)<R^{Q}
$$

Fix now $p \in E_{h}^{\alpha} \cap K$ and define $\delta_{h}=\left(\frac{4 C \mathscr{L}^{n}\left(E_{h}\right)}{\alpha}\right)^{1 / Q} ;$ then

$$
\frac{\mathscr{L}^{n}\left(E_{h} \cap B\left(p, \delta_{h}\right)\right)}{\mathscr{L}^{n}\left(B\left(p, \delta_{h}\right)\right)} \leq \frac{C \mathscr{L}^{n}\left(E_{h}\right)}{\delta_{h}^{Q}}=\frac{\alpha}{4} .
$$

On the other hand, by definition of $E_{h}^{\alpha}$ we can find arbitrarily small radii $r>0$ such that

$$
\frac{\mathscr{L}^{n}\left(E_{h} \cap B(p, r)\right)}{\mathscr{L}^{n}(B(p, r))} \geq \frac{\alpha}{2}
$$

Taking into account Proposition $\AA$.9. a continuity argument allows us to find $0<\varrho \leq \delta_{h}$ such that

$$
\mathscr{L}^{n}\left(E_{h} \cap B(x, \varrho)\right)=\frac{\alpha}{2} \mathscr{L}^{n}(B(x, \varrho)) .
$$

By the $5 r$-covering Lemma, we can find a family $\left\{B\left(p_{j}, \varrho_{j}\right): j \in \mathbb{N}\right\}$ of pairwise disjoint balls in $\Omega$ such that, for every $j \in \mathbb{N}$,

$$
\begin{aligned}
& p_{j} \in E_{h}^{\alpha} \cap K \\
& \mathscr{L}^{n}\left(E_{h} \cap B\left(p_{j}, \varrho_{j}\right)\right)=\frac{\alpha}{2} \mathscr{L}^{n}\left(B\left(p_{j}, \varrho_{j}\right)\right) \\
& E_{h}^{\alpha} \cap K \subseteq \bigcup_{j=0}^{\infty} B\left(p_{j}, 5 \varrho_{j}\right) .
\end{aligned}
$$

Since $\mathscr{L}^{n}\left(E_{h}\right)$ is finite, by Theorem 2.42 we get $M>0$ such that

$$
\frac{\alpha}{2 C} \varrho_{j}^{Q} \leq \frac{\alpha}{2} \mathscr{L}^{n}\left(B\left(p_{j}, \varrho_{j}\right)\right)=\mathscr{L}^{n}\left(E_{h} \cap B\left(p_{j}, \varrho_{j}\right)\right) \leq\left(M P_{X}\left(E_{h} ; B\left(p_{j}, \varrho_{j}\right)\right)\right)^{\frac{Q}{Q-1}} .
$$

Therefore we have that for every $j \in \mathbb{N}$

$$
\varrho_{j}^{Q-1} \leq M\left(\frac{2 C}{\alpha}\right)^{\frac{Q-1}{Q}} P_{X}\left(E_{h} ; B\left(p_{j}, \varrho_{j}\right)\right) .
$$

Finally

$$
\begin{aligned}
\mathscr{H}_{10 \delta_{h}}^{Q-1}\left(K \cap \bigcap_{i=0}^{\infty} E_{i}^{\alpha}\right) & \leq \mathscr{H}_{10 \delta_{h}}^{Q-1}\left(K \cap E_{h}^{\alpha}\right) \stackrel{(30)}{\leq} \omega_{Q-1} 5^{Q-1} \sum_{j=0}^{\infty} \varrho_{j}^{Q-1} \\
& \leq \omega_{Q-1} 5^{Q-1} M\left(\frac{2 C}{\alpha}\right)^{\frac{Q-1}{Q}} \sum_{j=0}^{\infty} P_{X}\left(E_{h} ; B\left(p_{j}, \varrho_{j}\right)\right) \\
& \leq \omega_{Q-1} 5^{Q-1} M\left(\frac{2 C}{\alpha}\right)^{\frac{Q-1}{Q}} P_{X}\left(E_{h} ; \Omega\right) .
\end{aligned}
$$

Taking the limit for $h \rightarrow \infty$ we get

$$
\mathscr{H}^{Q-1}\left(K \cap \bigcap_{i=0}^{\infty} E_{i}^{\alpha}\right)=0 .
$$

By the arbitrariness of $K$, the proof is complete. 
Before passing to the next result, we introduce some notation that we are going to use frequently in what follows. Let $p \in \mathbb{R}^{n}$ be fixed and let $F_{p}$ denote adapted exponential coordinates as in (41), for a fixed choice of a basis $Y_{1}, \ldots, Y_{n}$ as in (4). Given $r>0$ and $i \in\{1, \ldots, m\}$, define

$$
\widetilde{X}_{i}^{r}:=r\left(d \delta_{r^{-1}}\right)\left[\tilde{X}_{i} \circ \delta_{r}\right] .
$$

If $\widetilde{d}_{r}, \widetilde{B}_{r}(x, \varrho)$ denote, respectively, distance and balls with respect to the metric induced by the vector fields $\left(\widetilde{X}_{1}^{r}, \ldots, \widetilde{X}_{m}^{r}\right)$, it is easy to see that the dilations $\delta_{r}$ satisfy

$$
\widetilde{d}_{r}(\xi, \eta)=\frac{1}{r} \widetilde{d}\left(\delta_{r} \xi, \delta_{r} \eta\right)
$$

By Theorem 2.9, the convergence

$$
\lim _{r \rightarrow 0} \widetilde{B}_{r}(0, \varrho)=\widehat{B}(0, \varrho)
$$

holds in the Gromov-Hausdorff sense, $\widehat{B}(0, \varrho)$ denoting a ball in the tangent Carnot group at $p$. Moreover, given $u \in B V_{X, l o c}\left(\mathbb{R}^{n} ; \mathbb{R}^{k}\right)$ we set

$$
\widetilde{u}:=u \circ F_{p} \quad \text { and } \quad \widetilde{u}_{r}:=\widetilde{u} \circ \delta_{r} ;
$$

notice that $\left|D_{\widetilde{X}^{r}} \widetilde{u}_{r}\right|\left(\widetilde{B}_{r}(0, \varrho)\right)=r^{1-Q}\left|D_{\widetilde{X}} \widetilde{u}\right|(\widetilde{B}(0, r \varrho))$.

We can now prove the following lemma.

Lemma 3.2. Let $\left(\mathbb{R}^{n}, X\right)$ be an equiregular $C C$ space, let $\Omega \subseteq \mathbb{R}^{n}$ be open and consider $u \in$ $B V_{X}\left(\Omega ; \mathbb{R}^{k}\right)$. Then

$$
\mathscr{H}^{Q-1}\left(\left\{p \in \Omega: \limsup _{r \rightarrow 0} f_{B(p, r)}|u|^{\frac{Q}{Q-1}} d \mathscr{L}^{n}=+\infty\right\}\right)=0 .
$$

Proof. We can suppose without loss of generality that $k=1$. Possibly considering $|u|$ instead of $u$, we can suppose that $u \geq 0$; we also assume without loss of generality that $\Omega$ is bounded in $\mathbb{R}^{n}$. Define the set

$$
D=\left\{p \in \Omega: \limsup _{r \rightarrow 0} \frac{\left|D_{X} u\right|(B(p, r))}{r^{Q-1}}=+\infty\right\} .
$$

By Proposition A.4 we have that $\mathscr{H}^{Q-1}(D)=0$. For every $h \in \mathbb{N}$ we can find $t_{h} \in(h, h+1)$ such that

$$
P_{X}\left(\left\{u>t_{h}\right\}, \Omega\right) \leq \int_{h}^{h+1} P_{X}(\{u>t\}, \Omega) d t .
$$

Define $E_{h}=\left\{u>t_{h}\right\}$. Since $u \in L^{1}(\Omega)$ we have that $\lim _{h} \mathscr{L}^{n}\left(E_{h}\right)=0$ and applying the Coarea Formula of Theorem 2.40 we get

$$
\sum_{h=0}^{\infty} P_{X}\left(E_{h}, \Omega\right) \leq \int_{0}^{+\infty} P_{X}(\{u>t\}, \Omega) d t=\left|D_{X} u\right|(\Omega)<+\infty,
$$

and therefore $\lim _{h} P_{X}\left(E_{h}, \Omega\right)=0$. We are in a position to apply Lemma 3.1. Defining for every $h \in \mathbb{N}$

$$
F_{h}=\left\{p \in \Omega: \limsup _{r \rightarrow 0} \frac{\mathscr{L}^{n}\left(E_{h} \cap B(p, r)\right)}{\mathscr{L}^{n}(B(p, r))} \geq \alpha\right\},
$$

where $\alpha>0$ will be chosen later depending on $\Omega$ only, we have that $\mathscr{H}^{Q-1}\left(\bigcap_{h=0}^{\infty} F_{h}\right)=0$. It is then sufficient to prove the inclusion

$$
L:=\left\{p \in \Omega: \limsup _{r \rightarrow 0} f_{B(p, r)}|u|^{\frac{Q}{Q-1}} d \mathscr{L}^{n}=+\infty\right\} \subseteq D \cup \bigcap_{h=0}^{\infty} F_{h} .
$$


To this aim, we fix $p \notin D \cup \bigcap_{h=0}^{\infty} F_{h}$ and we prove that $p \notin L$. Define $u_{p, r}:=f_{B(p, r)} u d \mathscr{L}^{n}$. Applying Theorem 2.41 we get $C>0$ and $R>0$ such that for every $q \in \Omega$ and all $0<r<R$

$$
f_{B(q, r)}\left|u(y)-u_{q, r}\right|^{\frac{Q}{Q-1}} d \mathscr{L}^{n}(y) \leq C\left(\frac{\left|D_{X} u\right|(B(q, r))}{r^{Q-1}}\right)^{\frac{Q}{Q-1}} .
$$

It is enough to prove that $\lim _{\sup _{r \rightarrow 0}} u_{p, r}<+\infty$ : in this case, in fact, inequality (34) and the definition of $D$ would imply that $p \notin L$.

By contradiction we find an infinitesimal sequence $\left(r_{j}\right)$ such that $\lim _{j} u_{p, r_{j}}=+\infty$. Define $\widetilde{u}, \widetilde{u}_{r_{j}}$ as in (33) (with $r=r_{j}$ ) and $\widetilde{v}_{j}:=\widetilde{u}_{r_{j}}-u_{p, r_{j}}$; set also

$$
\widetilde{X}_{i}^{j}:=\widetilde{X}_{i}^{r_{j}} \quad \text { and } \quad \widetilde{X}^{j}:=\left(\widetilde{X}_{1}^{j}, \ldots, \widetilde{X}_{m}^{j}\right) .
$$

Since $p \notin D$, for any $\varrho>0$ the sequence $r_{j}^{1-Q}\left|D_{X} u\right|\left(B\left(p, \varrho r_{j}\right)\right)$ is uniformly bounded with respect to $j \in \mathbb{N}$; by Proposition 2.36, the same is true for the sequence

$$
\left|D_{\widetilde{X}^{j}} \widetilde{v}_{j}\right|\left(\widetilde{B}_{j}(0, \varrho)\right)=r_{j}^{1-Q}\left|D_{\widetilde{X}} \widetilde{u}\right|\left(\widetilde{B}\left(0, \varrho r_{j}\right)\right),
$$

where $\widetilde{B}_{j}(0, \varrho):=\widetilde{B}_{r_{j}}(0, \varrho)$ according to the notation introduced after (31). Taking also (32) into account, this proves that, for any compact set $K \subseteq \mathbb{R}^{n}$, the sequence $\left|D_{\widetilde{X}^{j}} \widetilde{v}_{j}\right|(K)$ is bounded; by (34), also $\left\|\widetilde{v}_{j}\right\|_{L^{1}(K)}$ is bounded.

By Theorem 2.43 (recalling also Theorem 2.7) there exists $w \in L^{1}(\widehat{B}(0,1))$ such that, possibly extracting a subsequence, $\widetilde{v}_{j} \rightarrow w$ in $L^{1}(\widehat{B}(0,1))$. Consequently, for almost every $x \in \widehat{B}(0,1)$ we have

$$
\lim _{j} u\left(F_{p}\left(\delta_{r_{j}} x\right)\right)=+\infty
$$

and then, for every $h \in \mathbb{N}$,

$$
\begin{aligned}
\mathscr{L}^{n}(\widehat{B}(0,1)) & =\lim _{j} \mathscr{L}^{n}\left(\left\{x \in \widetilde{B}_{j}(0,1): u\left(F_{p}\left(\delta_{r_{j}} x\right)\right)>t_{h}\right\}\right) \\
& =\lim _{j} r_{j}^{-Q} \mathscr{L}^{n}\left(\left\{x \in \widetilde{B}\left(0, r_{j}\right): u\left(F_{p}(x)\right)>t_{h}\right\}\right) \\
& =\lim _{j} \frac{1}{r_{j}^{Q}} \int_{B\left(p, r_{j}\right) \cap E_{h}}\left|\operatorname{det} \nabla F_{p}^{-1}\right| d \mathscr{L}^{n} \\
& \leq\left|\operatorname{det} \nabla F_{p}^{-1}(p)\right| \limsup _{r \rightarrow 0} \frac{\mathscr{L}^{n}\left(E_{h} \cap B(p, r)\right)}{\mathscr{L}^{n}(B(p, r))} \frac{\mathscr{L}^{n}(B(p, r))}{r^{Q}} \\
& \leq \frac{C}{\left|\operatorname{det} \nabla F_{p}(0)\right|} \limsup _{r \rightarrow 0} \frac{\mathscr{L}^{n}\left(E_{h} \cap B(p, r)\right)}{\mathscr{L}^{n}(B(p, r))}
\end{aligned}
$$

where $C>0$ is given by Theorem 2.2 with $K=\bar{\Omega}$. Notice that $\mathscr{L}^{n}(\widehat{B}(0,1))$ depends on $p$. Using (32) we obtain

$$
\begin{aligned}
& \limsup _{r \rightarrow 0} \frac{\mathscr{L}^{n}\left(E_{h} \cap B(p, r)\right)}{\mathscr{L}^{n}(B(p, r))} \\
\geq & \frac{\left|\operatorname{det} \nabla F_{p}(0)\right|}{C} \mathscr{L}^{n}(\widehat{B}(0,1))=\frac{\left|\operatorname{det} \nabla F_{p}(0)\right|}{C} \lim _{r \rightarrow 0} \mathscr{L}^{n}\left(\widetilde{B}_{r}(0,1)\right) \\
= & \frac{\left|\operatorname{det} \nabla F_{p}(0)\right|}{C} \lim _{r \rightarrow 0} \frac{1}{r^{Q}} \mathscr{L}^{n}(\widetilde{B}(0, r))=\frac{\left|\operatorname{det} \nabla F_{p}(0)\right|}{C} \lim _{r \rightarrow 0} \frac{1}{r^{Q}} \int_{B(p, r)}\left|\operatorname{det} \nabla F_{p}^{-1}\right| d \mathscr{L}^{n} \\
\geq & \frac{1}{C} \liminf _{r \rightarrow 0} \frac{\mathscr{L}^{n}(B(p, r))}{r^{Q}} \geq \frac{1}{C^{2}} .
\end{aligned}
$$

This proves that $p \in \bigcap_{h=0}^{\infty} F_{h}$ for $\alpha:=1 / C^{2}$, a contradiction. 
The following proposition contains some of the first "fine" properties of $B V_{X}$ functions we are interested in.

Proposition 3.3. Let $\left(\mathbb{R}^{n}, X\right)$ be an equiregular $C C$ space. Then there exists $\lambda: \mathbb{R}^{n} \rightarrow(0,+\infty)$ locally bounded away from 0 such that, for every open set $\Omega \subseteq \mathbb{R}^{n}$ and every $u \in B V_{X}\left(\Omega ; \mathbb{R}^{k}\right)$

$$
\left|D_{X} u\right| \geq \lambda\left|u^{+}-u^{-}\right| \mathscr{S}^{Q-1} \mathbf{L} \mathcal{J}_{u}
$$

and for every Borel set $B \subseteq \Omega$ the following implications hold:

$$
\begin{aligned}
& \mathscr{H}^{Q-1}(B)=0 \quad \Rightarrow \quad\left|D_{X} u\right|(B)=0 ; \\
& \mathscr{H}^{Q-1}(B)<+\infty \text { and } B \cap \mathcal{S}_{u}=\emptyset \quad \Rightarrow \quad\left|D_{X} u\right|(B)=0 .
\end{aligned}
$$

Proof. Let us prove the first part of the statement; we assume without loss of generality that $k=1$. Consider $p \in \mathcal{J}_{u}$. By Proposition 2.26 the sequence $\widetilde{u}_{r}:=u \circ F_{p} \circ \delta_{r}$ converges in $L^{1}(\widehat{B}(0,1))$ as $r \rightarrow 0$ to the function

$$
w_{p}(y):= \begin{cases}u^{+}(p) & \text { if } \widetilde{L}_{\nu(p)}(y) \geq 0 \\ u^{-}(p) & \text { if } \widetilde{L}_{\nu(p)}(y)<0 .\end{cases}
$$

Defining $\widetilde{X}_{i}^{r}$ as in (31) and using Propositions 2.44 and 2.36 we obtain for any positive $\varepsilon$ that

$$
\begin{aligned}
\liminf _{r \rightarrow 0} \frac{\left|D_{X} u\right|(B(p, r))}{r^{Q-1}} & \geq\left|\operatorname{det} \nabla F_{p}(0)\right| \liminf _{r}\left|D_{\widetilde{X}^{r}} \widetilde{u}_{r}\right|\left(\widetilde{B}_{r}(0,1)\right) \\
& \geq\left|\operatorname{det} \nabla F_{p}(0)\right| \liminf _{r}\left|D_{\widetilde{X}^{r}} \widetilde{u}_{r}\right|(\widehat{B}(0,1-\varepsilon)) \\
& \geq\left|\operatorname{det} \nabla F_{p}(0)\right|\left|D_{\widehat{X}} w_{p}\right|(\widehat{B}(0,1-\varepsilon)),
\end{aligned}
$$

whence

$$
\begin{aligned}
\liminf _{r \rightarrow 0} \frac{\left|D_{X} u\right|(B(p, r))}{r^{Q-1}} & \geq\left|\operatorname{det} \nabla F_{p}(0)\right|\left|D_{\widehat{X}} w_{p}\right|(\widehat{B}(0,1)) \\
& \geq\left|\operatorname{det} \nabla F_{p}(0)\right|\left|u^{+}(p)-u^{-}(p)\right| \mathscr{H}_{e}^{n-1}\left(\bar{\nu}^{\perp} \cap \widehat{B}(0,1)\right)
\end{aligned}
$$

where $\bar{\nu}:=\left(\nu_{1}, \ldots, \nu_{m}, 0, \ldots, 0\right) \in \mathbb{R}^{n}$ and $\mathscr{H}_{e}^{n-1}$ denotes the Euclidean Hausdorff measure in $\mathbb{R}^{n}$. It is easily seen that, for any $p \in \mathbb{R}^{n}$, there exist $c>0$ and a neighborhood $U$ of $p$ such that the function $\lambda(q):=\left|\operatorname{det} \nabla F_{q}(0)\right| \mathscr{H}_{e}^{n-1}\left(\bar{\nu}^{\perp} \cap \widehat{B}_{q}(0,1)\right)$ is such that $\lambda \geq c$ on $U$. By Corollary A.5. this proves the first part of the statement.

By Theorem 2.39, the implication (35) is true in case $k=1$ and $u=\chi_{E}$ for some $E \subseteq \mathbb{R}^{n}$ with finite $X$-perimeter. If $k=1$ and $u \in B V_{X}(\Omega)$, we define $E_{s}:=\{u>s\}$ and we apply Theorem 2.40 (and, again, Theorem 2.39) to get

$$
\left|D_{X} u\right|(B)=\int_{-\infty}^{+\infty} P_{X}\left(E_{s} ; B\right) d s=\int_{-\infty}^{+\infty}\left(\int_{B \cap \partial^{*} E_{s}} \theta_{s} d \mathscr{H}^{Q-1}\right) d s
$$

for suitable positive functions $\theta_{s}$. This allows to infer (35). In the general case $k \geq 1$, it is sufficient to recall inequality (25).

In order to prove (36) we consider $u \in B V_{X}\left(\Omega ; \mathbb{R}^{k}\right)$ and a Borel subset $B$ of $\Omega$ such that $B \cap \mathcal{S}_{u}=\emptyset$. If $k=1$, by Theorem 2.40 we obtain again

$$
\left|D_{X} u\right|(B)=\int_{-\infty}^{+\infty}\left(\int_{B \cap \partial^{*} E_{s}} \theta_{s} d \mathscr{H}^{Q-1}\right) d s=\int_{B} \int_{\mathbb{R}} \theta_{s}(p) \chi_{\partial^{*} E_{s}}(p) d s d \mathscr{H}^{Q-1}(p)=0,
$$

the last equality following from Proposition 2.22 . In the case $u \in B V_{X}\left(\Omega ; \mathbb{R}^{k}\right)$ with $k \geq 2$, it is sufficient to notice that $B \cap \mathcal{S}_{u}=\emptyset$ implies $B \cap \mathcal{S}_{u^{\alpha}}=\emptyset$ for every $\alpha=1, \ldots, k$, and one concludes using inequality (25). 
We now prove some of our main results.

Proof of Theorems 1.2 and 1.5. It is not restrictive to suppose $k=1$. We first prove Theorem 1.2.

By the Coarea Formula we get a countable and dense set $D \subseteq \mathbb{R}$ such that for every $t \in D$ the level set $\{u>t\}$ has finite $X$-perimeter. We prove that

$$
\mathcal{S}_{u} \backslash L \subseteq \bigcup_{t \in D} \partial^{*}\{u>t\}
$$

where, as in Lemma $3.2, L$ denotes the $\mathscr{H}^{Q-1}$-negligible set

$$
\left\{p \in \Omega: \limsup _{r \rightarrow 0} f_{B(p, r)}|u|^{\frac{Q}{Q-1}} d \mathscr{L}^{n}=+\infty\right\} .
$$

Theorem 1.2 is immediately implied by formula (38). In order to prove the latter, take $p \notin L$ and suppose that $p \notin \bigcup_{t \in D} \partial^{*}\{u>t\}$; we will prove that $p \notin \mathcal{S}_{u}$. By definition, $p$ is either a point of density 1 or a point of density 0 in $\{u>t\}$ for every $t \in D$. Notice that for every $t \in D \cap(0,+\infty)$ one has

$$
\frac{\mathscr{L}^{n}(\{u>t\} \cap B(p, r))}{\mathscr{L}^{n}(B(p, r))} \leq \frac{1}{t} f_{B(p, r)}|u| d \mathscr{L}^{n} \leq \frac{1}{t}\left(f_{B(p, r)}|u|^{\frac{Q}{Q-1}} d \mathscr{L}^{n}\right)^{\frac{Q-1}{Q}}
$$

and therefore, if $t \in D \cap(0,+\infty)$ is large enough, $p$ is a point of density 0 for $\{u>t\}$. Analogously, if $t \in D \cap(-\infty, 0)$ and $-t$ is large enough, $p$ is a point of density 1 for $\{u>t\}$. Hence we can find a real number

$$
z=z(p):=\sup \{t \in D:\{u>t\} \text { has density } 1 \text { at } p\} .
$$

By the density of $D$ in $\mathbb{R}$ we get that, for every $t>z,\{u>t\}$ has density 0 at $p$ and, for every $t<z,\{u>t\}$ has density 1 at $p$.

We prove now that $z$ is the approximate limit of $u$ at $p$. To this end define $E_{\varepsilon}:=\{|u-z|>\varepsilon\}$ and estimate

$$
\begin{aligned}
\frac{1}{r^{Q}} \int_{B(p, r)}|u-z| d \mathscr{L}^{n} & \leq \varepsilon C+\frac{1}{r^{Q}} \int_{E_{\varepsilon} \cap B(p, r)}|u-z| d \mathscr{L}^{n} \\
& \leq \varepsilon C+\frac{1}{r^{Q}}\left(\mathscr{L}^{n}\left(E_{\varepsilon} \cap B(p, r)\right)\right)^{1 / Q}\left(\int_{B(p, r)}|u-z|^{\frac{Q}{Q-1}} d \mathscr{L}^{n}\right)^{\frac{Q-1}{Q}} \\
& =\varepsilon C+\left(\frac{\mathscr{L}^{n}\left(E_{\varepsilon} \cap B(p, r)\right)}{r^{Q}}\right)^{1 / Q}\left(\frac{1}{r^{Q}} \int_{B(p, r)}|u-z|^{\frac{Q}{Q-1}} d \mathscr{L}^{n}\right)^{\frac{Q-1}{Q}}
\end{aligned}
$$

Since both $\{u>z+\varepsilon\}$ and $\{u<z-\varepsilon\}$ have density 0 at $p$, one has

$$
\lim _{r \rightarrow 0} \frac{\mathscr{L}^{n}\left(E_{\varepsilon} \cap B(p, r)\right)}{r^{Q}}=0
$$

and, since $p \notin L$, we get

$$
\limsup _{r \rightarrow 0} \frac{1}{r^{Q}} \int_{B(p, r)}|u-z| d \mathscr{L}^{n} \leq C \varepsilon,
$$

from which we deduce that $p \notin \mathcal{S}_{u}$, as desired.

We now prove Theorem 1.5. When property $\mathcal{R}$ holds, the countable $X$-rectifiability of $\mathcal{S}_{u}$ immediately follows from (38). We have to prove that $\mathscr{H}^{Q-1}\left(\mathcal{S}_{u} \backslash \mathcal{J}_{u}\right)=0$. Let $\nu=\nu_{\mathcal{S}_{u}}$ be the 
horizontal normal to $\mathcal{S}_{u}$ and recall the notation $B_{\nu}^{ \pm}(p, r)$ introduced in (15). By Proposition 3.7 below, for $\mathscr{H}^{Q-1}$-almost every $p \in \mathcal{S}_{u}$ there exist $u^{+}(p)$ and $u^{-}(p)$ in $\mathbb{R}^{k}$ such that

$$
\lim _{r \rightarrow 0} \frac{1}{r^{Q}} \int_{B_{\nu(p)}^{+}(p, r)}\left|u-u^{+}(p)\right| d \mathscr{L}^{n}=\lim _{r \rightarrow 0} \frac{1}{r^{Q}} \int_{B_{\nu(p)}^{-}(p, r)}\left|u-u^{-}(p)\right| d \mathscr{L}^{n}=0 .
$$

Notice that $u^{+}(p) \neq u^{-}(p)$, for otherwise $u$ would have an approximate limit at $p$. This implies that $p$ is an approximate $X$-jump point associated with the triple $\left(u^{+}(p), u^{-}(p), \nu(p)\right)$, and this concludes the proof.

A milder version of Theorem 1.5 holds when $\left(\mathbb{R}^{n}, X\right)$ satisfies the weaker property $\mathcal{L} \mathcal{R}$, that we now introduce.

Definition 3.4 (Property $\mathcal{L} \mathcal{R}$ ). Let $\left(\mathbb{R}^{n}, X\right)$ be an equiregular $\mathrm{CC}$ space with homogeneous dimension $Q \in \mathbb{N}$. We say that $\left(\mathbb{R}^{n}, X\right)$ satisfies the property $\mathcal{L} \mathcal{R}$ if, for every open set $\Omega \subseteq \mathbb{R}^{n}$ and every $E \subseteq \mathbb{R}^{n}$ with locally finite $X$-perimeter in $\Omega$, the essential boundary $\partial^{*} E \cap \Omega$ is countably $X$-Lipschitz rectifiable.

The proof of the following result is an immediate consequence of (38).

Theorem 3.5. Let $\left(\mathbb{R}^{n}, X\right)$ be an equiregular $C C$ space satisfying property $\mathcal{L} \mathcal{R}$ and let $u \in$ $B V_{X}\left(\Omega ; \mathbb{R}^{k}\right)$. Then $\mathcal{S}_{u}$ is countably $X$-Lipschitz rectifiable.

Before proving Proposition 3.7, that we used in the proof of Theorem 1.5, we state the following theorem, which is a consequence of some results contained in [46]. We use the notation

$$
B_{f}^{ \pm}(p, r):=\{q \in B(p, r): \pm f(q)>0\} .
$$

Theorem 3.6. Let $\left(\mathbb{R}^{n}, X\right)$ be an equiregular $C C$ space, let $\Omega \subseteq \mathbb{R}^{n}$ be an open set and let $f \in C_{X}^{1}(\Omega)$ be such that $X f \neq 0$ on $\Omega$; let $S$ be the $C_{X}^{1}$ hypersurface $S:=\{p \in \Omega: f(p)=0\}$. Then there exist linear operators $T^{+}, T^{-}: B V_{X, l o c}\left(\Omega ; \mathbb{R}^{k}\right) \rightarrow L_{l o c}^{1}\left(S, \mathscr{H}^{Q-1}\right)$ such that, for any $u \in B V_{X, l o c}\left(\Omega ; \mathbb{R}^{k}\right)$, one has for $\mathscr{H}^{Q-1}$-a.e. $p \in S$

$$
\lim _{r \rightarrow 0} \frac{1}{r^{Q}} \int_{B_{f}^{+}(p, r)}\left|u-T^{+} u(p)\right| d \mathscr{L}^{n}=\lim _{r \rightarrow 0} \frac{1}{r^{Q}} \int_{B_{f}^{-}(p, r)}\left|u-T^{-} u(p)\right| d \mathscr{L}^{n}=0 .
$$

In particular, for $\mathscr{H}^{Q-1}$-a.e. $p \in S$

$$
T^{ \pm} u(p)=\lim _{r \rightarrow 0} \frac{1}{r^{Q}} \int_{B_{f}^{ \pm}(p, r)} u d \mathscr{L}^{n} .
$$

We can now prove the following proposition, where we implicitly use Remark 2.27.

Proposition 3.7. Let $\left(\mathbb{R}^{n}, X\right)$ be an equiregular $C C$ space and let $\Omega \subseteq \mathbb{R}^{n}$ be an open set. Let $R \subseteq \Omega$ be a countably $X$-rectifiable set with horizontal normal $\nu_{R}$. Then, for every $u \in$ $B V_{X}\left(\Omega ; \mathbb{R}^{k}\right)$ and for $\mathscr{H}^{Q-1}$-almost every $p \in R$ there exists a couple $\left(u_{R}^{+}(p), u_{R}^{-}(p)\right) \in \mathbb{R}^{k} \times \mathbb{R}^{k}$ such that

$$
\lim _{r \rightarrow 0} \frac{1}{r^{Q}} \int_{\Omega \cap B_{\nu_{R}(p)}^{+}(p, r)}\left|u-u_{R}^{+}(p)\right| d \mathscr{L}^{n}=\lim _{r \rightarrow 0} \frac{1}{r^{Q}} \int_{\Omega \cap B_{\nu_{R}(p)}^{-}(p, r)}\left|u-u_{R}^{-}(p)\right| d \mathscr{L}^{n}=0 .
$$

Moreover, if $\left(\mathbb{R}^{n}, X\right)$ satisfies property $\mathcal{R}$ and $R=\mathcal{J}_{u}^{3}$, then $\left(u_{\mathcal{J}_{u}}^{+}(p), u_{\mathcal{J}_{u}}^{-}(p), \nu_{\mathcal{J}_{u}}(p)\right)$ is an approximate $X$-jump triple for $u$ at $p$ in the sense of Definition 2.24.

\footnotetext{
${ }^{3}$ The jump set $\mathcal{J}_{u}$ is countably $X$-rectifiable by Theorem 1.5 and Remark 2.25 .
} 
Proof. We can assume without loss of generality that $k=1$. Let $u \in B V_{X}(\Omega)$ be fixed. By definition of countable $X$-rectifiability we can find a family $\left\{S_{i}: i \in \mathbb{N}\right\}$ of $C_{X}^{1}$ hypersurfaces in $\mathbb{R}^{n}$ such that

$$
\mathscr{H}^{Q-1}\left(R \backslash \bigcup_{i=0}^{\infty} S_{i}\right)=0 .
$$

For every $i \in \mathbb{N}$ we can write, at least locally, $S_{i}=\left\{f_{i}=0\right\}$ and we can suppose that $X f_{i} \neq 0$ on $S_{i}$. Formula (39) easily follows (with $u_{R}^{ \pm}(p)=T^{ \pm} u(p)$ ) from Theorem 3.6 for $\mathscr{H}^{Q-1}$-a.e. $p \in R$ such that $\#\left\{i \in \mathbb{N}: p \in S_{i}\right\}=1$. It is then enough to show that, for any fixed couple $i, j \in \mathbb{N}$ with $i \neq j$ and for $\mathscr{H}^{Q-1}$-almost every $p \in S_{i} \cap S_{j}$, the equivalence

$$
\left(T_{i}^{+} u(p), T_{i}^{-} u(p), \nu_{S_{i}}(p)\right) \equiv\left(T_{j}^{+} u(p), T_{j}^{-} u(p), \nu_{S_{j}}(p)\right)
$$

holds. Here, $T_{i}^{ \pm}, T_{j}^{ \pm}$are the trace operators provided by Theorem 3.6 with $f=f_{i}, f_{j}$.

Fix a point $p \in S_{i} \cap S_{j}$ where $\nu_{S_{i}}(p)= \pm \nu_{S_{j}}(p)$; recall that this fact occurs at $\mathscr{H}^{Q-1}$-a.e. $p \in S_{i} \cap S_{j}$. Assume that $\nu_{S_{i}}(p)=\nu_{S_{j}}(p)$, i.e., $\frac{X f_{i}(p)}{\left|X f_{i}(p)\right|}=\frac{X f_{j}(p)}{\left|X f_{j}(p)\right|}$; by Theorem 3.6 we have for $\mathscr{H}^{Q-1}$-a.e. such $p$ that

$$
\begin{aligned}
\left|T_{i}^{ \pm}(p)-T_{j}^{ \pm}(p)\right| & =\lim _{r \rightarrow 0} \frac{1}{r^{Q}}\left|\int_{\left\{ \pm f_{i}>0\right\} \cap B(p, r)} u d \mathscr{L}^{n}-\int_{\left\{ \pm f_{j}>0\right\} \cap B(p, r)} u d \mathscr{L}^{n}\right| \\
& \leq \lim _{r \rightarrow 0} \frac{1}{r^{Q}} \int_{\left\{f_{i} f_{j} \leq 0\right\} \cap B(p, r)}|u| d \mathscr{L}^{n} \\
& \leq \lim _{r \rightarrow 0} \frac{1}{r^{Q}} \mathscr{L}^{n}\left(\left\{f_{i} f_{j} \leq 0\right\} \cap B(p, r)\right)^{1 / Q}\left(\int_{B(p, r)}|u|^{\frac{Q}{Q-1}} d \mathscr{L}^{n}\right)^{\frac{Q-1}{Q}} .
\end{aligned}
$$

By Remark 2.15 we have

$$
\lim _{r \rightarrow 0} \frac{1}{r^{Q}} \mathscr{L}^{n}\left(\left\{f_{i} f_{j} \leq 0\right\} \cap B(p, r)\right)=0,
$$

while by Lemma 3.2 we also have that for $\mathscr{H}^{Q-1}$-almost every $p \in \Omega$

$$
\limsup _{r \rightarrow 0} \frac{1}{r^{Q}} \int_{B(p, r)}|u|^{\frac{Q}{Q-1}} d \mathscr{L}^{n}<+\infty .
$$

This proves that $T_{i}^{ \pm}(p)=T_{j}^{ \pm}(p)$ for $\mathscr{H}^{Q-1}$-a.e. $p \in S_{i} \cap S_{j}$ such that $\nu_{S_{i}}(p)=\nu_{S_{j}}(p)$. A similar argument shows that $T_{i}^{ \pm}(p)=T_{j}^{\mp}(p)$ holds for $\mathscr{H}^{Q-1}$-a.e. $p \in S_{i} \cap S_{j}$ with $\nu_{S_{i}}(p)=-\nu_{S_{j}}(p)$. This proves (40), while the last statement of the proposition follows from Theorem 3.6.

The problem of studying "intrinsic" measures of submanifolds of a CC space goes back to M. Gromov [26, 0.6.b]: the interested reader might consult [32, 33, 34, 40] and the references therein. Since we do not intend to dwell on such questions, we follow a different ("axiomatic") path; this is based on the following definition, where we chose to work with the spherical Hausdorff measure $\mathscr{S}^{Q-1}$, rather than the standard one, because the results mentioned above (as well as [22, 23]) suggest $\mathscr{S}^{Q-1}$ to be more natural than the standard measure $\mathscr{H}^{Q-1}$.

Definition 3.8 (Property $\mathcal{D}$ ). Let $\left(\mathbb{R}^{n}, X\right)$ be an equiregular CC space with homogeneous dimension $Q \in \mathbb{N}$. We say that $\left(\mathbb{R}^{n}, X\right)$ satisfies the property $\mathcal{D}$ if there exists a function $\zeta: \mathbb{R} \times \mathbb{S}^{m-1} \rightarrow(0,+\infty)$ such that, for every $C_{X}^{1}$ hypersurface $S \subseteq \mathbb{R}^{n}$ and every $p \in S$, one has

$$
\lim _{r \rightarrow 0} \frac{\mathscr{S}^{Q-1}(S \cap B(p, r))}{r^{Q-1}}=\zeta\left(p, \nu_{S}(p)\right)
$$


Remark 3.9. If $\left(\mathbb{R}^{n}, X\right)$ is an equiregular $\mathrm{CC}$ space satisfying property $\mathcal{D}$ and $R \subseteq \mathbb{R}^{n}$ is $X$-rectifiable, then we have

$$
\lim _{r \rightarrow 0} \frac{\mathscr{S}^{Q-1}(R \cap B(p, r))}{r^{Q-1}}=\zeta\left(p, \nu_{R}(p)\right) \quad \text { for } \mathscr{S}^{Q-1} \text {-a.e. } p \in R,
$$

where $\zeta$ is as in Definition 3.8 .

Let us prove this fact. Let $S_{i}, i \in \mathbb{N}$, be a family of $C_{X}^{1}$ hypersurfaces such that $\mathscr{S}^{Q-1}(R \backslash$ $\left.\cup_{i \in \mathbb{N}} S_{i}\right)=0$; it is enough to show that, for any fixed $i \in \mathbb{N}$, we have

$$
\lim _{r \rightarrow 0} \frac{\mathscr{S}^{Q-1}(R \cap B(p, r))}{r^{Q-1}}=\zeta\left(p, \nu_{R}(p)\right) \quad \text { for } \mathscr{S}^{Q-1} \text {-a.e. } p \in R \cap S_{i} .
$$

Setting $R \Delta S_{i}:=\left(R \backslash S_{i}\right) \cup\left(S_{i} \backslash R\right.$ ), by Remark A.6 (applied with $\mu:=\mathscr{S}^{Q-1}\left\llcorner\left(R \Delta S_{i}\right)\right.$ ) we obtain

$$
\lim _{r \rightarrow 0} \frac{\mathscr{S}^{Q-1}\left(\left(R \Delta S_{i}\right) \cap B(p, r)\right)}{r^{Q-1}}=0 \quad \text { for } \mathscr{S}^{Q-1} \text {-a.e. } p \in R \cap S_{i},
$$

which gives for $\mathscr{S}^{Q-1}$-a.e. $p \in R \cap S_{i}$

$$
\lim _{r \rightarrow 0} \frac{\mathscr{S}^{Q-1}(R \cap B(p, r))}{r^{Q-1}}=\lim _{r \rightarrow 0} \frac{\mathscr{S}^{Q-1}\left(S_{i} \cap B(p, r)\right)}{r^{Q-1}}=\zeta\left(p, \nu_{S_{i}}(p)\right)=\zeta\left(p, \nu_{R}(p)\right)
$$

as desired.

Assuming properties $\mathcal{R}$ and $\mathcal{D}$ we are able to prove the following result, where we use the notation $u_{R}^{+}, u_{R}^{-}$of Proposition 3.7.

Theorem 3.10. Let $\left(\mathbb{R}^{n}, X\right)$ be an equiregular $C C$ space satisfying properties $\mathcal{R}$ and $\mathcal{D}$; then, there exists a function $\sigma: \mathbb{R}^{n} \times \mathbb{S}^{m-1} \rightarrow(0,+\infty)$ such that the following holds. For every open set $\Omega \subseteq \mathbb{R}^{n}, u \in B V_{X}\left(\Omega ; \mathbb{R}^{k}\right)$ and every countably $X$-rectifiable set $R \subseteq \mathbb{R}^{n}$ one has

$$
D_{X} u\left\llcorner R=\sigma\left(\cdot, \nu_{R}\right)\left(u_{R}^{+}-u_{R}^{-}\right) \otimes \nu_{R} \mathscr{S}^{Q-1}\llcorner R .\right.
$$

In particular, $D_{X}^{j} u=\sigma\left(\cdot, \nu_{u}\right)\left(u^{+}-u^{-}\right) \otimes \nu_{u} \mathscr{S}^{Q-1}\left\llcorner\mathcal{J}_{u}\right.$.

Proof. We can assume without loss of generality that $k=1$ and $\mathscr{S}^{Q-1}(R)<\infty$. By Theorem 1.5 and Proposition 3.3 we can also assume that $R \subseteq \mathcal{J}_{u}$. Given $p \in \mathbb{R}^{n}$ we work in adapted exponential coordinates $F_{p}$ around $p$ and we define

$$
\sigma(p, \nu):=\frac{\left|\operatorname{det} \nabla F_{p}(0)\right| \mathscr{H}_{e}^{n-1}\left(\bar{\nu}^{\perp} \cap \widehat{B}_{p}(0,1)\right)}{\zeta(p, \nu)}
$$

where $\zeta$ is as in Definition 3.8 and, as in the proof of Proposition 3.3 , $\mathscr{H}_{e}^{n-1}$ denotes the Euclidean Hausdorff measure in $\mathbb{R}^{n}$.

Let $\mu_{R}:=D_{X} u\left\llcorner R\right.$; by Proposition 3.3 we have $\mu_{R} \ll \mathscr{S}^{Q-1}\llcorner R$. By Remark 3.9] we can use [17, Theorem 2.9.8] (joint with [17, Theorem 2.8.17]) and it is enough to prove that for $\mathscr{S}^{Q-1}$-a.e. $p \in R$

$$
\lim _{r \rightarrow 0} \frac{\mu_{R}(B(p, r))}{\mathscr{S}^{Q-1}(R \cap B(p, r))}=\sigma\left(p, \nu_{R}(p)\right)\left(u_{R}^{+}(p)-u_{R}^{-}(p)\right) \nu_{R}(p) ;
$$

notice that the limit above exists $\mathscr{S}^{Q-1}$-almost everywhere. Taking into account Remark 3.9 and the fact that (by Remark A.6)

$$
\lim _{r \rightarrow 0} \frac{\left|D_{X} u-\mu_{R}\right|(B(p, r))}{r^{Q-1}}=0 \quad \text { for } \mathscr{S}^{Q-1} \text {-a.e. } p \in R
$$


it suffices to prove that, for $\mathscr{S}^{Q-1}$-a.e. $p \in R$, there exists an infinitesimal sequence $\left(r_{i}\right)$ such that

$$
\lim _{i \rightarrow+\infty} \frac{D_{X} u\left(B\left(p, r_{i}\right)\right)}{r_{i}^{Q-1}}=\left|\operatorname{det} \nabla F_{p}(0)\right| \mathscr{H}_{e}^{n-1}\left(\bar{\nu}^{\perp} \cap \widehat{B}_{p}(0,1)\right)\left(u_{R}^{+}(p)-u_{R}^{-}(p)\right) \nu_{R}(p) .
$$

We prove that such a sequence exists at all points where $\lim \sup _{r \rightarrow 0} \frac{\left|D_{X} u\right|(B(p, r))}{r^{Q-1}}<\infty$, which holds for $\mathscr{S}^{Q-1}$-a.e. $p \in R$ due to Remark A.6.

Let then such a $p \in R$ be fixed; since $R \subseteq \mathcal{J}_{u}$, the functions $\widetilde{u}_{r}:=u \circ F_{p} \circ \delta_{r}$ converge in $L_{l o c}^{1}\left(\mathbb{R}^{n}\right)$ to

$$
w_{p}(y):= \begin{cases}u^{+}(p) & \text { if } \widetilde{L}_{\nu_{R}(p)}(y) \geq 0 \\ u^{-}(p) & \text { if } \widetilde{L}_{\nu_{R}(p)}(y)<0\end{cases}
$$

where we used the fact that $\nu_{R}=\nu_{\mathcal{J}_{u}}=\nu_{u} \mathscr{S}^{Q-1}$-a.e. on $R$. Let $\widetilde{u}:=u \circ F_{p}$; since (recall notation (31) $)\left|D_{\widetilde{X}^{r}} \widetilde{u}_{r}\right|\left(\widetilde{B}_{r}(0, \varrho)\right)=\left|D_{\widetilde{X}} \widetilde{u}\right|(\widetilde{B}(0, r \varrho)) / r^{Q-1}$ is bounded as $r \rightarrow 0$ for any positive $\varrho$, by Remark 2.45 the sequence $D_{\widetilde{X}^{r}} \widetilde{u}_{r}$ weakly* converges in $\mathbb{R}^{n}$ to $D_{\widehat{X}} w_{p}$ as $r \rightarrow 0$. Let $s_{i}$ be an infinitesimal sequence such that $\left|D_{\widetilde{X}^{s_{i}}} \widetilde{u}_{s_{i}}\right|$ weakly* to some measure $\lambda$ in $\mathbb{R}^{n}$; let $\varrho \in(0,1)$ be such that $\lambda\left(\partial \widehat{B}_{p}(0, \varrho)\right)=0$ (which holds for all except at most countably many $\varrho$ ) and define $r_{i}:=\varrho s_{i}$. Proposition 2.36 gives

$$
\begin{aligned}
\lim _{i \rightarrow \infty} \frac{D_{X} u\left(B\left(p, r_{i}\right)\right)}{r_{i}^{Q-1}} & =\left|\operatorname{det} \nabla F_{p}(0)\right| \lim _{i \rightarrow \infty} \frac{D_{\widetilde{X}} \widetilde{u}\left(\widetilde{B}\left(0, r_{i}\right)\right)}{r_{i}^{Q-1}} \\
& =\left|\operatorname{det} \nabla F_{p}(0)\right| \lim _{i \rightarrow \infty} \frac{D_{\widetilde{X}^{s}} \widetilde{u}^{s_{i}}\left(\widetilde{B}_{s_{i}}(0, \varrho)\right)}{\varrho^{Q-1}} .
\end{aligned}
$$

We prove in a moment that

$$
\lim _{i \rightarrow \infty} \frac{D_{\widetilde{X}^{s_{i}}} \widetilde{u}^{s_{i}}\left(\widetilde{B}_{s_{i}}(0, \varrho)\right)}{\varrho^{Q-1}}=\frac{D_{\widehat{X}} w_{p}\left(\widehat{B}_{p}(0, \varrho)\right)}{\varrho^{Q-1}}
$$

assuming this to be true, we have

$$
\begin{aligned}
\lim _{i \rightarrow \infty} \frac{D_{X} u\left(B\left(p, r_{i}\right)\right)}{r_{i}^{Q-1}} & =\left|\operatorname{det} \nabla F_{p}(0)\right| \frac{D_{\widehat{X}} w_{p}\left(\widehat{B}_{p}(0, \varrho)\right)}{\varrho^{Q-1}} \\
& =\left|\operatorname{det} \nabla F_{p}(0)\right| \mathscr{H}_{e}^{n-1}\left(\bar{\nu}^{\perp} \cap \widehat{B}_{p}(0,1)\right)\left(u_{R}^{+}(p)-u_{R}^{-}(p)\right) \nu_{R}(p) .
\end{aligned}
$$

and the proof would be concluded.

Let us prove (41). Defining

$$
\mu_{i}:=D_{\widetilde{X}^{s_{i}}} \widetilde{u}^{s_{i}}\left\llcorner\widetilde{B}_{s_{i}}(0, \varrho), \quad \mu:=D_{\widehat{X}} w_{p}\left\llcorner\widehat{B}_{p}(0, \varrho)\right.\right.
$$

and taking into account [2, Proposition 1.62 (b)], it will suffice to show that

$$
\mu_{i} \stackrel{*}{\rightarrow} \mu \quad \text { and } \quad\left|\mu_{i}\right| \stackrel{*}{\rightarrow} \lambda \mathrm{L} \widehat{B}_{p}(0, \varrho) .
$$

Concerning the first statement in (42), fix a test function $\varphi \in C_{c}^{0}\left(\mathbb{R}^{n}\right)$; then

$$
\begin{aligned}
& \lim _{i \rightarrow \infty} \int \varphi d \mu_{i}=\lim _{i \rightarrow \infty} \int_{\widetilde{B}_{s_{i}}(0, \varrho)} \varphi d D_{\widetilde{X}^{s_{i}}} \widetilde{u}^{s_{i}} \\
= & \lim _{i \rightarrow \infty} \int_{\widehat{B}_{p}(0, \varrho)} \varphi d D_{\widetilde{X}^{s_{i}}} \widetilde{u}^{s_{i}}+\int_{\widetilde{B}_{s_{i}}(0, \varrho) \backslash \widehat{B}_{p}(0, \varrho)} \varphi d D_{\widetilde{X}^{s_{i}}} \widetilde{u}^{s_{i}}-\int_{\widehat{B}_{p}(0, \varrho) \backslash \widetilde{B}_{s_{i}}(0, \varrho)} \varphi d D_{\widetilde{X}^{s_{i}}} \widetilde{u}^{s_{i}} \\
= & \lim _{i \rightarrow \infty} \int_{\widehat{B}_{p}(0, \varrho)} \varphi d D_{\widehat{X}^{\prime}} w_{p},
\end{aligned}
$$


where the last equality follows from the weak* convergence of $D_{\widetilde{X}^{s_{i}}} \widetilde{u}^{s_{i}}$ to $D_{\widehat{X}} w_{p}$ and the fact that (denoting by $\Delta$ the symmetric difference of sets)

$$
\lim _{i \rightarrow \infty}\left|D_{\widetilde{X}^{s_{i}}} \widetilde{u}^{s_{i}}\right|\left(\widetilde{B}_{s_{i}}(0, \varrho) \Delta \widehat{B}_{p}(0, \varrho)\right)=0
$$

that, in turn, can be proved as follows. For any $\varepsilon>0$ there exists $\delta \in(0, \varrho)$ such that

$$
\lambda\left(\overline{\widehat{B}_{p}(0, \varrho+\delta)} \backslash \widehat{B}_{p}(0, \varrho-\delta)\right)<\varepsilon
$$

by Theorem 2.9 we obtain

$$
\begin{aligned}
\limsup _{i \rightarrow \infty}\left|D_{\widetilde{X}^{s_{i}}} \widetilde{u}^{s_{i}}\right|\left(\widetilde{B}_{s_{i}}(0, \varrho) \Delta \widehat{B}_{p}(0, \varrho)\right) & \leq \limsup _{i \rightarrow \infty}\left|D_{\widetilde{X}^{s_{i}}} \widetilde{u}^{s_{i}}\right|\left(\overline{\widehat{B}_{p}(0, \varrho+\delta)} \backslash \widehat{B}_{p}(0, \varrho-\delta)\right) \\
& \leq \lambda\left(\widehat{\widehat{B}_{p}(0, \varrho+\delta)} \backslash \widehat{B}_{p}(0, \varrho-\delta)\right)<\varepsilon
\end{aligned}
$$

where we used [2, Proposition $1.62(\mathrm{a})]$.

The first statement in (42) is proved; we are left with the second one, which can be easily proved by the very same argument taking into account that $\left|\mu_{i}\right|=\left|D_{\widetilde{X}^{s}} \widetilde{u}^{s_{i}}\right|\left\llcorner\widetilde{B}_{s_{i}}(0, \varrho)\right.$.

Let us recall once more the notation $u_{p, r}:=f_{B(p, r)} u d \mathscr{L}^{n}$.

Lemma 3.11. Let $\left(\mathbb{R}^{n}, X\right)$ be an equiregular $C C$ space of homogeneous dimension $Q$ and let $\Omega \subseteq \mathbb{R}^{n}$ be an open bounded set. Then there exist $C=C(\Omega)>0$ and $R=R(\Omega)>0$ such that, for every $p \in \Omega$, every $u \in B V_{X}\left(\Omega ; \mathbb{R}^{k}\right)$ and every $0<r<\min \left\{R, \frac{1}{2} d(p, \partial \Omega)\right\}$, one has

$$
\left|u_{p, 2 r}-u_{p, r}\right| \leq C r^{1-Q}\left|D_{X} u\right|(B(p, 2 r)) .
$$

Proof. We use Theorems 2.2 and 2.41 to estimate

$$
\begin{aligned}
\left|u_{p, 2 r}-u_{p, r}\right| & =\left|f_{B(p, r)}\left(u-u_{p, 2 r}\right) d \mathscr{L}^{n}\right| \leq C f_{B(p, 2 r)}\left|u-u_{p, 2 r}\right| d \mathscr{L}^{n} \\
& \leq C\left(f_{B(p, 2 r)}\left|u-u_{p, 2 r}\right|^{\frac{Q}{Q-1}} d \mathscr{L}^{n}\right)^{\frac{Q-1}{Q}} \leq C r^{1-Q}\left|D_{X} u\right|(B(p, 2 r)) .
\end{aligned}
$$

As mentioned in the Introduction, the next lemma possesses its own interest and it is the key tool in the proof of Theorem 1.1.

Lemma 3.12. Let $\left(\mathbb{R}^{n}, X\right)$ be an equiregular $C C$ space of homogeneous dimension $Q$ and let $\Omega \subseteq \mathbb{R}^{n}$ be an open bounded set. Then there exist $C=C(\Omega)>0$ and $R=R(\Omega)>0$ such that the following holds: for every $u \in B V_{X}\left(\Omega ; \mathbb{R}^{k}\right), p \in \Omega \backslash \mathcal{S}_{u}$ and $0<r<\min \left\{R, \frac{1}{2} d(p, \partial \Omega)\right\}$ one has

$$
\int_{B(p, r)} \frac{\left|u(q)-u^{\star}(p)\right|}{d(p, q)} d \mathscr{L}^{n}(q) \leq C\left(\left|D_{X} u\right|(B(p, r))+\int_{0}^{1} \frac{\left|D_{X} u\right|(B(p, t r))}{t^{Q}} d t\right) .
$$

In particular

$$
\int_{B(p, r)} \frac{\left|u(q)-u^{\star}(p)\right|}{d(p, q)} d \mathscr{L}^{n}(q) \leq C \int_{0}^{2} \frac{\left|D_{X} u\right|(B(p, t r))}{t^{Q}} d t .
$$


Proof. Let $u, p, r$ be as in the statement; we introduce the compact notation $u_{i}:=u_{p, 2^{-i} r}, i \in \mathbb{N}$. Since $u_{i} \rightarrow u^{\star}(p)$ as $i \rightarrow \infty$ we estimate

$$
\begin{aligned}
& \int_{B(p, r)} \frac{\left|u(q)-u^{\star}(p)\right|}{d(p, q)} d \mathscr{L}^{n}(q) \\
\leq & \sum_{i=1}^{\infty} \int_{B\left(p, 2^{-i+1} r\right) \backslash B\left(p, 2^{-i} r\right)} \frac{\left|u(q)-u^{\star}(p)\right|}{2^{-i} r} d \mathscr{L}^{n}(q) \\
\leq & \sum_{i=1}^{\infty} \frac{2^{i}}{r} \int_{B\left(p, 2^{-i+1} r\right) \backslash B\left(p, 2^{-i} r\right)}\left(\left|u(q)-u_{i-1}\right|+\sum_{j=i-1}^{\infty}\left|u_{j}-u_{j+1}\right|\right) d \mathscr{L}^{n}(q)
\end{aligned}
$$

and use Lemma 3.11 and Theorem 2.41 to get

$$
\begin{aligned}
& \leq C \sum_{i=1}^{\infty} \frac{2^{i}}{r}\left(2^{-i} r\left|D_{X} u\right|\left(B\left(p, 2^{1-i} r\right)\right)+\sum_{j=i-1}^{\infty}\left(2^{1-i} r\right)^{Q}\left(2^{-(j+1)} r\right)^{1-Q}\left|D_{X} u\right|\left(B\left(p, 2^{-j} r\right)\right)\right) \\
& \leq C \sum_{i=1}^{\infty}\left(\left|D_{X} u\right|\left(B\left(p, 2^{1-i} r\right)\right)+\sum_{j=i-1}^{\infty} 2^{(j-i+1)(Q-1)}\left|D_{X} u\right|\left(B\left(p, 2^{-j} r\right)\right)\right) \\
& =C \sum_{k=0}^{\infty}\left(1+1+2^{Q-1}+\left(2^{Q-1}\right)^{2}+\cdots+\left(2^{Q-1}\right)^{k}\right)\left|D_{X} u\right|\left(B\left(p, 2^{-k} r\right)\right) \\
& \leq C \sum_{k=0}^{\infty} \frac{2^{(k+1)(Q-1)}-1}{2^{Q-1}-1}\left|D_{X} u\right|\left(B\left(p, 2^{-k} r\right)\right) .
\end{aligned}
$$

Since $Q \geq 2$ we have $2^{Q-1}-1 \geq \frac{2^{Q-1}}{2}$, hence

$$
\begin{aligned}
\int_{B(p, r)} \frac{\left|u(q)-u^{\star}(p)\right|}{d(p, q)} d \mathscr{L}^{n}(q) & \leq C \sum_{k=0}^{\infty} 2^{k(Q-1)}\left|D_{X} u\right|\left(B\left(p, 2^{-k} r\right)\right) \\
& =C\left(\left|D_{X} u\right|(B(p, r))+\sum_{k=1}^{\infty} 2^{k(Q-1)}\left|D_{X} u\right|\left(B\left(p, 2^{-k} r\right)\right)\right) \\
& =C\left(\left|D_{X} u\right|(B(p, r))+\sum_{k=1}^{\infty} \int_{2^{-k}}^{2^{1-k}} 2^{k Q}\left|D_{X} u\right|\left(B\left(p, 2^{-k} r\right)\right) d t\right) \\
& \leq C\left(\left|D_{X} u\right|(B(p, r))+\sum_{k=1}^{\infty} \int_{2^{-k}}^{2^{1-k}} \frac{\left|D_{X} u\right|(B(p, t r))}{t^{Q}} d t\right) \\
& =C\left(\left|D_{X} u\right|(B(p, r))+\int_{0}^{1} \frac{\left|D_{X} u\right|(B(p, t r))}{t^{Q}} d t\right),
\end{aligned}
$$

as desired.

We can now prove one of our main results; recall that we denote by $D_{X}^{a p} u(p)$ the approximate $X$-gradient of $u$ at $p$.

Proof of Theorem 1.1. We can assume without loss of generality that $k=1$. Suppose that $D_{X} u=v \mathscr{L}^{n}+D_{X}^{s} u$ is the Radon-Nykodým decomposition of the measure $D_{X} u$ with respect to $\mathscr{L}^{n}$. By the Radon-Nykodým Theorem in doubling metric spaces (see e.g. [43, Theorem 4.7 and Remark 4.5]), at $\mathscr{L}^{n}$-almost every $p \in \Omega$ we have

$$
\lim _{r \rightarrow 0} \frac{D_{X}^{s} u(B(p, r))}{r^{Q}}=0 .
$$


It is sufficient to prove that, for every $p \in \Omega \backslash\left(\mathcal{S}_{u} \cup \mathcal{S}_{v}\right)$ for which (43) holds, $u$ is approximately $X$-differentiable at $p$ with $D_{X}^{a p} u(p)=v^{\star}(p)$.

Let $R>0$ and $f \in C^{1}(B(p, R))$ be such that $f(p)=0$ and $X f(p)=v^{\star}(p)$ and define

$$
w(q):=u(q)-u^{\star}(p)-f(q)
$$

Then $w \in B V_{X}(B(p, R)), p \notin \mathcal{S}_{w}$ and $w^{\star}(p)=0$. We are in a position to apply Lemma 3.12 to the function $w$ and get $C>0$ so that, for small enough $r$,

$$
\begin{aligned}
\frac{1}{r^{Q}} \int_{B(p, r)} \frac{\left|u(q)-u^{\star}(p)-f(q)\right|}{d(p, q)} d \mathscr{L}^{n}(q) & \leq \frac{C}{r^{Q}} \int_{0}^{2} \frac{\left|D_{X} w\right|(B(p, t r))}{t^{Q}} d t \\
& \leq C \sup _{t \in(0,2)} \frac{\left|D_{X} w\right|(B(p, t r))}{(t r)^{Q}}
\end{aligned}
$$

It is then enough to show that $\lim _{r \rightarrow 0} r^{-Q}\left|D_{X} w\right|(B(p, r))=0$. Taking into account that $D_{X} w=(v-X f) \mathscr{L}^{n}+D_{X}^{s} u$ and (43), it suffices to check that

$$
\lim _{r \rightarrow 0} \frac{1}{r^{Q}} \int_{B(p, r)}|v-X f| d \mathscr{L}^{n}=0
$$

which follows by the generalized Lebesgue's differentiation theorem (see e.g. [28, Section 2.7]) and the inequality $|v-X f| \leq\left|v-v^{\star}(p)\right|+\left|v^{\star}(p)-X f\right|$.

As for classical $B V$ functions (see e.g. [2, pag. 177], the (approximate) convergence of $u \in B V_{X}$ to $u^{\star}(p)$ at points $p \notin \mathcal{S}_{u}$ can be improved in a $L^{1^{*}}$-sense, as we now state.

Proposition 3.13. Let $\left(\mathbb{R}^{n}, X\right)$ be an equiregular $C C$ space, $\Omega \subseteq \mathbb{R}^{n}$ an open set and let $u \in B V_{X}(\Omega)$. Then

$$
\lim _{r \rightarrow 0} f_{B(p, r)}\left|u-u^{\star}(p)\right|^{\frac{Q}{Q-1}} d \mathscr{L}^{n}=0 \quad \text { for } \mathscr{H}^{Q-1} \text {-a.e. } p \in \Omega \backslash \mathcal{S}_{u} .
$$

Proof. We first prove that

$$
\lim _{r \rightarrow 0} \frac{\left|D_{X} u\right|(B(p, r))}{r^{Q-1}}=0 \quad \text { for } \mathscr{H}^{Q-1} \text {-a.e. } p \in \Omega \backslash \mathcal{S}_{u} .
$$

Let $t>0$ be fixed and consider the set

$$
E_{t}:=\left\{p \in \Omega \backslash \mathcal{S}_{u}: \limsup _{r \rightarrow 0} \frac{\left|D_{X} u\right|(B(p, r))}{r^{Q-1}}>t\right\} .
$$

By Proposition A.4 one has $\mathscr{H}^{Q-1}\left(E_{t}\right)<\infty$; Proposition 3.3 then implies that $\left|D_{X} u\right|\left(E_{t}\right)=0$ and again Proposition A.4 gives $\mathscr{H}^{Q-1}\left(E_{t}\right)=0$. Since this is true for all positive $t$, formula (44) immediately follows.

Combining Theorem 2.41 and (44) we immediately get that for $\mathscr{H}^{Q-1}$-a.e. $p \in \Omega$

$$
\lim _{r \rightarrow 0} f_{B(p, r)}\left|u-u_{p, r}\right|^{\frac{Q}{Q-1}} d \mathscr{L}^{n}=0 .
$$

The conclusion follows by

$$
\left|u-u^{\star}(p)\right|^{\frac{Q}{Q-1}} \leq 2^{\frac{1}{Q-1}}\left(\left|u_{p, r}-u^{\star}(p)\right|^{\frac{Q}{Q-1}}+\left|u-u_{p, r}\right|^{\frac{Q}{Q-1}}\right) .
$$

together with $u^{\star}(p)=\lim _{r \rightarrow 0} u_{p, r}$. 
When $\left(\mathbb{R}^{n}, X\right)$ satisfies property $\mathcal{R}, \Omega \subseteq \mathbb{R}^{n}$ is open and $u \in B V_{X}\left(\Omega, \mathbb{R}^{k}\right)$, by Theorem 1.5 the precise representative $u^{\mathfrak{p}}$

$$
u^{\mathfrak{p}}(p):= \begin{cases}u^{\star}(p) & \text { if } p \in \Omega \backslash \mathcal{S}_{u} \\ \frac{u^{+}(p)+u^{-}(p)}{2} & \text { if } p \in \mathcal{J}_{u}\end{cases}
$$

is defined $\mathscr{H}^{Q-1}$-a.e. on $\Omega$. We have the following result.

Theorem 3.14. Let $\left(\mathbb{R}^{n}, X\right)$ be an equiregular $C C$ space satisfying property $\mathcal{R}, \Omega \subseteq \mathbb{R}^{n}$ an open set and let $u \in B V_{X}\left(\Omega ; \mathbb{R}^{k}\right)$. Then

$$
\lim _{r \rightarrow 0} f_{B(p, r)} u d \mathscr{L}^{n}=u^{\mathfrak{p}}(p) \quad \text { for } \mathscr{H}^{Q-1} \text {-a.e. } p \in \Omega .
$$

Proof. The statement easily follows for $\mathscr{H}^{Q-1}$-a.e. $p \in \Omega \backslash \mathcal{S}_{u}$ by Proposition 3.13. By Theorem 1.5 it suffices to prove the statement for all $p \in \mathcal{J}_{u}$, which directly follows from Proposition 2.23 and Definition 2.24.

Remark 3.15. When $\left(\mathbb{R}^{n}, X\right)$ satisfies property $\mathcal{R}$, then $D_{X}^{c} u=D_{X}^{s} u\left\llcorner\left(\Omega \backslash \mathcal{S}_{u}\right)\right.$ : to see this, it is enough to combine Proposition 3.3 and Theorem 1.5 .

We now want to study the properties of the decomposition $D_{X} u=D_{X}^{a} u+D_{X}^{c} u+D_{X}^{j} u$; recall that $\mathscr{H}_{e}^{1}$ denotes the Euclidean Hausdorff measure in $\mathbb{R}^{n}$.

Theorem 3.16 (Properties of Cantor part and jump part). Let $u \in B V_{X}\left(\Omega ; \mathbb{R}^{k}\right)$. Then the following facts hold:

(a) $D_{X}^{a} u=D_{X} u\left\llcorner(\Omega \backslash S)\right.$ and $D_{X}^{s} u=D_{X} u\llcorner S$, where

$$
S:=\left\{p \in \Omega: \lim _{r \rightarrow 0} \frac{\left|D_{X} u\right|(B(p, r))}{r^{Q}}=+\infty\right\} .
$$

Moreover, if $E \subseteq \mathbb{R}^{k}$ is such that $\mathscr{H}_{e}^{1}(E)=0$, then $D_{X}^{a p} u=0 \mathscr{L}^{n}$-a.e. in $\left(u^{\star}\right)^{-1}(E)$.

(b) Let $\Theta_{u} \subseteq S$ be defined by

$$
\Theta_{u}:=\left\{p \in \Omega: L(p):=\liminf _{r \rightarrow 0} \frac{\left|D_{X} u\right|(B(p, r))}{r^{Q-1}}>0\right\} .
$$

Then $\mathcal{J}_{u} \subseteq \Theta_{u}$.

Moreover, if $\left(\mathbb{R}^{n}, X\right)$ satisfies property $\mathcal{R}$, then

(c) $\mathscr{H}^{Q-1}\left(\Theta_{u} \backslash \mathcal{J}_{u}\right)=0$ and $D_{X}^{j} u=D_{X} u\left\llcorner\Theta_{u}\right.$. More generally, for every Borel set $\Sigma$ containing $\mathcal{J}_{u}$ and $\sigma$-finite with respect to $\mathscr{H}^{Q-1}$ we have $D_{X}^{j} u=D_{X} u\llcorner\Sigma$.

(d) $D_{X}^{c} u=D_{X} u\left\llcorner\left(S \backslash \Theta_{u}\right)\right.$.

(e) if $B \subseteq \Omega$ is such that either $\mathscr{H}^{Q-1}\left\llcorner B\right.$ is $\sigma$-finite or $B=\left(u^{\star}\right)^{-1}(E)$ for some $\mathscr{H}_{e}^{1}$ negligible set $E \subseteq \mathbb{R}^{k}$, then $D_{X}^{c} u(B)=0$.

Proof. In order to prove the first part of statement (a) it is sufficient to apply Radon-Nykodým Theorem in doubling metric spaces (see e.g. [43, Theorem 4.7 and Remark 4.5]). Concerning the second part, assume first that $k=1$ and let $B:=\left(u^{\star}\right)^{-1}(E)$. By Proposition 2.22, for any $t \notin E$ we have $B \cap \partial^{*}\{u>t\}=\emptyset$. By Theorems 2.40 and 2.39 we obtain

$$
\left|D_{X} u\right|(B)=\int_{\mathbb{R}} P_{X}(\{u>t\} \cap B) d t=0=\int_{\mathbb{R} \backslash E} \int_{\partial^{*}\{u>t\} \cap B} \theta_{t} d \mathscr{H}^{Q-1} d t=0,
$$


where $\theta_{t}$ denote suitable positive functions. When $k \geq 1$ and $j=1, \ldots, k$ we set $E_{j}:=\{t \in \mathbb{R}$ : $t=z_{j}$ for some $\left.z \in E\right\}$; the set $E_{j}$ is such that $\mathscr{L}^{1}\left(E_{j}\right)=0$ and by (25)

$$
\left|D_{X} u\right|(B) \leq \sum_{j=1}^{k}\left|D_{X} u^{j}\right|(B) \leq \sum_{j=1}^{k}\left|D_{X} u^{j}\right|\left(\left(\left(u^{j}\right)^{\star}\right)^{-1}\left(E_{j}\right)\right)=0 .
$$

We then conclude by Theorem 1.1 .

By (37) in the proof of Proposition 3.3 we have $\mathcal{J}_{u} \subseteq \Theta_{u}$, and statement (b) follows.

We now prove (c). Applying Proposition A.4 we get that for every $h \in \mathbb{N} \backslash\{0\}$

$$
\left|D_{X} u\right|\left\llcorner\left\{L \geq \frac{1}{h}\right\} \geq \frac{1}{h} \omega_{Q-1} \mathscr{H}^{Q-1}\left\llcorner\left\{L \geq \frac{1}{h}\right\},\right.\right.
$$

where $L$ is defined in statement (b). In particular $\mathscr{H}^{Q-1}\left(\left\{L \geq \frac{1}{h}\right\}\right)<+\infty$. By (36)

$$
\left|D_{X} u\right|\left(\left\{L \geq \frac{1}{h}\right\} \backslash \mathcal{S}_{u}\right)=0
$$

and consequently (by (46) $)$ also $\mathscr{H}^{Q-1}\left(\left\{L \geq \frac{1}{h}\right\} \backslash \mathcal{S}_{u}\right)=0$. Since $\left\{L \geq \frac{1}{h}\right\} \nearrow \Theta_{u}$, on passing to the limit for $h \rightarrow+\infty$ we get $\mathscr{H}^{Q-1}\left(\Theta_{u} \backslash \mathcal{S}_{u}\right)=0$. Taking Theorem 1.5 into account, we conclude that $\mathscr{H}^{Q-1}\left(\Theta_{u} \backslash \mathcal{J}_{u}\right)=0$.

Let now $\Sigma$ be as in statement (c). Then, taking into account Proposition 3.3 and the fact that $\mathscr{H}^{Q-1}\left(\mathcal{S}_{u} \backslash \mathcal{J}_{u}\right)=0$, we have

$$
\begin{aligned}
D_{X} u\llcorner\Sigma & =D_{X} u\left\llcorner\mathcal{J}_{u}+D_{X} u\left\llcorner\left(\Sigma \backslash \mathcal{J}_{u}\right)\right.\right. \\
& =D_{X}^{j} u+D_{X} u\left\llcorner\left(\Sigma \backslash \mathcal{S}_{u}\right)+D_{X} u\left\llcorner\left(\Sigma \cap \mathcal{S}_{u} \backslash \mathcal{J}_{u}\right)\right.\right. \\
& =D_{X}^{j} u+D_{X} u\left\llcorner\left(\Sigma \backslash \mathcal{S}_{u}\right) .\right.
\end{aligned}
$$

Since $\Sigma$ is $\sigma$-finite with respect to $\mathscr{H}^{Q-1}$, using (36) we get that $D_{X} u\left\llcorner\left(\Sigma \backslash \mathcal{S}_{u}\right)=0\right.$, and so $D_{X} u\left\llcorner\Sigma=D_{X}^{j} u\right.$.

Statement $(d)$ follows from (a), (b), (c) and the decomposition $D_{X} u=D_{X}^{a} u+D_{X}^{c} u+D_{X}^{j} u$, which immediately give that $D_{X}^{c} u=D_{X} u\left\llcorner\left(S \backslash \Theta_{u}\right)\right.$.

We prove (e) in case $\mathscr{H}^{Q-1} \mathrm{~L} B$ is $\sigma$-finite; we can assume (see e.g. [2, Theorem 1.43]) that $B$ is a Borel set. Using Proposition 3.3 and Theorem 1.5 we get that $\left|D_{X} u\right|\left(B \backslash \mathcal{J}_{u}\right)=0$, which gives $\left(D_{X}^{a} u+D_{X}^{c} u\right)\llcorner B=0$.

Concerning the second part of statement $(e)$, suppose first that $k=1$ and let $B=\left(u^{\star}\right)^{-1}(E)$ with $\mathscr{L}^{1}(E)=0$. By Proposition 2.22 we know that $\partial^{*}\{u>t\} \cap B=\emptyset$ for every $t \notin E$. Applying the Coarea Formula of Theorem 2.40 we get

$$
\left|D_{X} u\right|(B)=\int_{E} \int_{\partial^{*}\{u>t\} \cap B} \theta_{t} d \mathscr{H}^{Q-1} d t=0
$$

for suitable functions $\theta_{t}$. In the general case $k \geq 2$ define for every $\alpha=1, \ldots, k$ the sets $E_{\alpha}:=\pi_{\alpha}(E)$, where $\pi_{\alpha}$ denotes the canonical projection $\pi_{\alpha}\left(x_{1}, \ldots, x_{k}\right)=x_{\alpha}$. Noticing that $\mathscr{L}^{1}\left(E_{\alpha}\right) \leq \mathscr{H}_{e}^{1}(E)=0$, we can use (25) to estimate

$$
\left|D_{X} u\right|\left(\left(u^{\star}\right)^{-1}(E)\right) \leq \sum_{\alpha=1}^{k}\left|D_{X} u^{\alpha}\right|\left(\left(u^{\star}\right)^{-1}(E)\right) \leq \sum_{\alpha=1}^{k}\left|D_{X} u^{\alpha}\right|\left(\left(\left(u^{\alpha}\right)^{\star}\right)^{-1}\left(E_{\alpha}\right)\right)=0 .
$$




\section{Applications to some Classes of Carnot groups}

Some of the main results of this paper rely on properties $\mathcal{R}, \mathcal{L} \mathcal{R}$ or $\mathcal{D}$; in this section we show how they can be in some meaningful CC spaces and, in particular, in some large classes of Carnot groups.

We start by introducing the reduced boundary $\mathcal{F}_{X} E$ of a set $E$ with finite $X$-perimeter. Recall that the reduced boundary was the object originally considered by E. De Giorgi in the seminal paper [13] about the rectifiability of sets with finite (Euclidean) perimeter in $\mathbb{R}^{n}$.

Definition 4.1 (Reduced boundary). Let $E \subseteq \mathbb{R}^{n}$ be a set with locally finite $X$-perimeter. The $X$-reduced boundary $\mathcal{F}_{X} E$ of $E$ is the set of points $p \in \mathbb{R}^{n}$ such that $P_{X}(E, B(p, r))>0$ for any $r>0$ and the limit

exists with $\left|\widetilde{\nu}_{E}(p)\right|=1$.

$$
\widetilde{\nu}_{E}(p):=\lim _{r \rightarrow 0} \frac{D_{X} \chi_{E}(B(p, r))}{\left|D_{X} \chi_{E}\right|(B(p, r))}
$$

For sets with finite (Euclidean) perimeter in $\mathbb{R}^{n}$ the symmetric difference between the essential boundary and the reduced one is $\mathscr{H}_{e}^{n-1}$-negligible, see e.g. [2, Theorem 3.61]. In our setting we have the following result, which is a known consequence of Theorem 2.39, see e.g. [22, Theorem 7.3] for the Heisenberg group case and [23, Lemma 2.26] for step 2 Carnot groups.

Theorem 4.2. Let $\left(\mathbb{R}^{n}, X\right)$ be an equiregular $C C$ space of homogeneous dimension $Q$ and let $E \subseteq \mathbb{R}^{n}$ be a set of locally finite $X$-perimeter. Then $\mathscr{H}^{Q-1}\left(\partial^{*} E \backslash \mathcal{F}_{X} E\right)=0$.

Proof. By Theorem 2.39 we have $D_{X} \chi_{E}=\theta \nu_{E} \mathscr{H}^{Q-1}\left\llcorner\partial^{*} E\right.$ for a suitable positive function $\theta$. Therefore it is enough to prove that, for $\mathscr{H}^{Q-1}$-almost every $p \in \partial^{*} E$, one has

$$
\lim _{r \rightarrow 0} \frac{D_{X} \chi_{E}(B(p, r))}{\left|D \chi_{E}\right|(B(p, r))}=\nu_{E}(p) .
$$

This fact directly follows from [17, Theorem 2.9.8] taking into account Theorem 2.39] and [17, Theorem 2.8.17].

The proof of Theorem 4.2 also shows that $\widetilde{\nu}_{E}=\nu_{E} \mathscr{H}^{Q-1}$-a.e. on $\mathcal{F}_{X} E$.

The papers [22, 23, 35] prove the countable $X$-rectifiability of the reduced boundary of sets with locally finite $X$-perimeter in, respectively, Heisenberg groups, Carnot groups of step 2, and Carnot groups of type $\star$. These results, in conjunction with Theorem 4.2, show that property $\mathcal{R}$ is satisfied in these settings.

Actually, Theorem 4.2 and the results about blow-up and representation of the $X$-perimeter available in Heisenberg groups ([22, Theorems 4.1 and 7.1]), step 2 Carnot groups ([23, Theorems 3.1 and 3.9]) and Carnot groups of type $\star$ [35. Theorems 4.12 and 4.13] imply that also property $\mathcal{D}$ is satisfied in these settings.

Using also the left-invariance of the structure we can conclude what follows.

Theorem 4.3. Heisenberg groups, Carnot groups of step 2 and Carnot groups of type $\star$ satisfy properties $\mathcal{R}$ and $\mathcal{D}$. In particular, Theorems 1.5, 1.6. 1.7 and 3.14 hold in these settings.

Moreover, the function $\sigma(p, \nu)$ appearing in 1.7 and 3.14 does not depend on the point $p \in \mathbb{R}^{n}$.

In the paper [14 the class of Carnot groups $\mathbb{G}$ satisfying the following assumption (see e.g. [37] for the notion of abnormal curve)

there exists at least one direction $V$ in the first layer of the stratified Lie algebra of $\mathbb{G}$ such that $t \mapsto \exp (t V)$ is not an abnormal curve

is considered. This class includes, for instance, the Engel group, which is the simplest example where the rectifiability problem for sets with finite $X$-perimeter is open. One of the main 
results of [14] is the following one: for any set $E$ with finite $X$-perimeter in a Carnot group $\mathbb{G}$ satisfying (47), the reduced boundary $\mathcal{F}_{X} E$ is countably $X$-Lipschitz rectifiable. Together with Theorem 4.2, this gives the following result.

Theorem 4.4. The property $\mathcal{L} \mathcal{R}$ is satisfied in all Carnot groups $\mathbb{G}$ such that (47) holds; in particular, Theorem 3.5 holds in such groups.

\section{Appendix A. Some tools from Geometric Measure Theory in metric spaces.}

Proposition A.1. Let $u \in L_{\text {loc }}^{1}\left(\Omega ; \mathbb{R}^{k}\right)$. If $p \in \Omega \backslash \mathcal{S}_{u}$, then, for any $\varepsilon>0$, the set

$$
E_{\varepsilon}:=\left\{q \in \Omega:\left|u(q)-u^{\star}(p)\right|>\varepsilon\right\}
$$

has density 0 at $p$. Conversely, if $u \in L_{\text {loc }}^{\infty}\left(\Omega ; \mathbb{R}^{k}\right)$ and $z \in \mathbb{R}^{k}$ are such that, for any $\varepsilon>0$, the set

$$
E_{\varepsilon}:=\{q \in \Omega:|u(q)-z|>\varepsilon\}
$$

has density 0 at $p$, then $p \in \Omega \backslash \mathcal{S}_{u}$ and $z=u^{\star}(p)$.

In particular, if $k=1$ and $p \in \Omega \backslash \mathcal{S}_{u}$ and $t \neq u^{\star}(p)$, then $p \notin \partial^{*}\{q \in \Omega: u(q)>t\}$.

Proof. Suppose $p \in \Omega \backslash \mathcal{S}_{u}$. By Chebychev inequality we have

$$
\varepsilon \frac{\mathscr{L}^{n}\left(E_{\varepsilon} \cap B(p, r)\right)}{\mathscr{L}^{n}(B(p, r))} \leq f_{B(p, r)}\left|u-u^{\star}(p)\right| d \mathscr{L}^{n} \rightarrow 0 \quad \text { as } r \rightarrow 0 .
$$

Conversely, suppose that $u$ and $z$ are as in the statement. Then we have for any $r \in(0,1)$

$$
f_{B(p, r)}|u-z| d \mathscr{L}^{n} \leq\left(\|u\|_{L^{\infty}\left(B(p, 1) ; \mathbb{R}^{k}\right)}+|z|\right) \frac{\left.\mathscr{L}^{n}\left(B(p, r) \cap E_{\varepsilon}\right)\right)}{\mathscr{L}^{n}(B(p, r))}+\varepsilon \frac{\left.\mathscr{L}^{n}\left(B(p, r) \backslash E_{\varepsilon}\right)\right)}{\mathscr{L}^{n}(B(p, r))},
$$

which is infinitesimal as $r \rightarrow 0$.

Finally, consider $p \in \Omega \backslash \mathcal{S}_{u}$ and let $t \neq u^{\star}(p)$. We already know that both $\left\{u>u^{\star}(p)+\varepsilon\right\}$ and $\left\{u<u^{\star}(p)-\varepsilon\right\}$ have density 0 at $p$ for every $\varepsilon>0$. If $t>u^{\star}(p)$, then choosing $\varepsilon=$ $t-u^{\star}(p)$ we have that $\{u>t\}$ has density 0 at $p$. If $t<u^{\star}(p)$ then choose $\eta>0$ such that $\varepsilon=u^{\star}(p)-t-\eta>0$ to infer that $\{u<t+\eta\}$ has density 0 at $p$, and consequently $\{u \geq t+\eta\}$ has density 1 at $p$. This implies that also $\{u>t\}$ has density 1 at $p$.

The following result is classical, see e.g. [43] or [28].

Theorem A.2 (5r-Covering Lemma). Let $(M, d)$ be a separable metric space and let $\mathcal{B}$ a family of closed balls in $M$ such that

$$
\sup \{\operatorname{diam} B: B \in \mathcal{B}\}<+\infty .
$$

Denote by $5 B$ the closed metric ball with same center as $B$ and radius 5 times larger than that of $B$. Then there exists a countable and pairwise disjoint subfamily $\mathcal{F} \subseteq \mathcal{B}$ such that

$$
\bigcup \mathcal{B} \subseteq \bigcup_{B \in \mathcal{F}} 5 B
$$

Definition A.3 (Hausdorff measures). Let $(M, d)$ be a metric space and $k \geq 0$. For any $\delta>0$ and any $E \subseteq M$ we define

$$
\begin{aligned}
& \mathscr{H}_{\delta}^{k}(E):=\frac{\omega_{k}}{2^{k}} \inf \left\{\sum_{h=0}^{\infty}\left(\operatorname{diam} E_{h}\right)^{k}: E \subseteq \bigcup_{h=0}^{\infty} E_{h}, \operatorname{diam} E_{h}<\delta\right\} \\
& \mathscr{S}_{\delta}^{k}(E):=\frac{\omega_{k}}{2^{k}} \inf \left\{\sum_{h=0}^{\infty}\left(\operatorname{diam} B_{h}\right)^{k}: E \subseteq \bigcup_{h=0}^{\infty} B_{h}, B_{h} \text { balls with diam } B_{h}<\delta\right\},
\end{aligned}
$$


where $\omega_{\alpha}:=\pi^{\alpha / 2} \Gamma(1+\alpha / 2)^{-1}$ and $\Gamma(t):=\int_{0}^{+\infty} s^{t-1} e^{-s} d s$ is the Euler $\Gamma$ function. The Hausdorff measure $\mathscr{H}^{k}(E)$ and the spherical Hausdorff measure $\mathscr{S}^{k}(E)$ of $E$ are

$$
\begin{aligned}
& \mathscr{H}^{k}(E):=\sup _{\delta>0} \mathscr{H}_{\delta}^{k}(E)=\lim _{\delta \rightarrow 0} \mathscr{H}_{\delta}^{k}(E) \\
& \mathscr{S}^{k}(E):=\sup _{\delta>0} \mathscr{S}_{\delta}^{k}(E)=\lim _{\delta \rightarrow 0} \mathscr{S}_{\delta}^{k}(E) .
\end{aligned}
$$

The useful inequalities $\mathscr{H}^{k} \leq \mathscr{S}^{k} \leq 2^{k} \mathscr{H}^{k}$ are classical.

If $(M, d, \mu)$ is a metric measure space, $k \geq 0$ and $x \in M$, we define the upper $k$-density $\Theta_{k}^{*}(\mu, x)$ and the lower $k$-density $\Theta_{* k}(\mu, x)$ of $\mu$ at $x$ as

$$
\Theta_{k}^{*}(\mu, x):=\limsup _{r \rightarrow 0} \frac{\mu(B(x, r))}{\omega_{k} r^{k}}, \quad \Theta_{* k}(\mu, x):=\liminf _{r \rightarrow 0} \frac{\mu(B(x, r))}{\omega_{k} r^{k}} .
$$

For every Borel set $E \subseteq \mathbb{R}^{n}$ we will also write $\Theta_{k}^{*}(E, x):=\Theta_{k}^{*}\left(\mathscr{H}^{k}\llcorner E, x)\right.$ and $\Theta_{* k}(E, x):=$ $\Theta_{* k}\left(\mathscr{H}^{k}\llcorner E, x)\right.$. If $\Theta_{k}^{*}(\mu, x)=\Theta_{* k}(\mu, x)$, then the common value is denoted by $\Theta_{k}(\mu, x)$ and it will be called $k$-density of $\mu$ at $x$. Hausdorff measures and densities are linked by Propositions A.4 and A.5 below. A proof of Proposition A.4 can be found for instance in [43, Theorem 3.2]; in the latter reference, statement (i) below is stated with $\mathscr{H}^{k}$ in place of $\mathscr{S}^{k}$, but the careful reader will notice that the proof is indeed provided for this stronger version.

Proposition A.4. Let $(M, d)$ be a separable metric space, let $\mu$ be a Borel regular Radon measure on $M$, let $E \subseteq M$ be a Borel set and let $t>0$. Then the following facts hold.

(i) If $\Theta_{k}^{*}(\mu, x) \geq t$ for every $x \in E$, then $\mu \geq t \mathscr{S}^{k}\llcorner E$.

(ii) If $\Theta_{k}^{*}(\mu, x) \leq t$ for every $x \in E$, then $\mu \leq 2^{k} t \mathscr{H}^{k}\llcorner E$.

In particular, for $\mathscr{H}^{k}$-almost every $x \in \mathbb{R}^{n}$ we have $\Theta_{k}^{*}(\mu, x)<+\infty$.

Corollary A.5. Let $(M, d)$ be a separable metric space, let $\mu$ be a Borel regular Radon measure on $M$, let $E \subseteq M$ be a Borel set and let $f: E \rightarrow \mathbb{R}$ be a strictly positive function. Then the following facts hold.

(i) If $\Theta_{k}^{*}(\mu, x) \geq f(x)$ for every $x \in E$, then $\mu \geq f \mathscr{S}^{k} \mathrm{~L} E$.

(ii) If $\Theta_{k}^{*}(\mu, x) \leq f(x)$ for every $x \in E$, then $\mu \leq 2^{k} f \mathscr{H}^{k}\llcorner E$.

Proof. (i) Let $\varepsilon>0$ and define for every $j \in \mathbb{Z}$ the set

$$
E_{j}:=\left\{x \in E:(1+\varepsilon)^{j}<f(x) \leq(1+\varepsilon)^{j+1}\right\} .
$$

Suppose that $\Theta_{k}^{*}(\mu, x) \geq f(x)$ for every $x \in E$. Then, using (i) of Proposition A.4 we get

$$
\mu=\sum_{j \in \mathbb{Z}} \mu\left\llcorner E_{j} \geq \sum_{j \in \mathbb{Z}}(1+\varepsilon)^{j} \mathscr{S}^{k}\left\llcorner E_{j} \geq \sum_{j \in \mathbb{Z}} \frac{f}{1+\varepsilon} \mathscr{S}^{k}\left\llcorner E_{j}=\frac{f}{1+\varepsilon} \mathscr{S}^{k}\llcorner E .\right.\right.\right.
$$

The statement follows by the arbitrariness of $\varepsilon$.

(ii) Using (ii) of Proposition A.4 we have

$$
\begin{aligned}
\mu & =\sum_{j \in \mathbb{Z}} \mu\left\llcorner E_{j} \leq \sum_{j \in \mathbb{Z}} 2^{k}(1+\varepsilon)^{j+1} \mathscr{H}^{k}\left\llcorner E_{j}\right.\right. \\
& \leq \sum_{j \in \mathbb{Z}} 2^{k}(1+\varepsilon) f \mathscr{S}^{k}\left\llcorner E_{j}=2^{k}(1+\varepsilon) f \mathscr{S}^{k}\llcorner E .\right.
\end{aligned}
$$

The statement follows by the arbitrariness of $\varepsilon$.

As a consequence of the Corollary A.5 we have the following remark. 
Remark A.6. Under the same assumptions of Corollary A.5, for $\mathscr{H}^{k}$-almost every $x \in \mathbb{R}^{n}$ we have $\Theta_{k}^{*}(\mu, x)<+\infty$ and for any Borel set $B \subseteq \mathbb{R}^{n}$ the implication

$$
\mu(B)=0 \quad \Longrightarrow \quad \Theta_{k}(\mu, x)=0 \text { for } \mathscr{H}^{k} \text {-a.e. } x \in B
$$

holds. In particular, if $\mu=g \mathscr{H}^{k}\left\llcorner E\right.$ we have $\Theta_{k}(\mu, x)=0$ for $\mathscr{H}^{k}$-almost every $x \in \mathbb{R}^{n} \backslash E$.

Definition A.7 (Porous sets). Let $(M, d)$ be a metric space and let $E \subseteq M$ be a Borel set. Then $E$ is said to be porous if there esist $\alpha \in(0,1)$ and $R>0$ such that for every $x \in M$ and every $r \in(0, R)$ there exists $y \in M$ such that

$$
B(y, \alpha r) \subseteq B(x, r) \backslash E .
$$

Proposition A.8. Let $(M, d)$ be a locally compact and separable metric space, $\mu$ a doubling Radon measure on $M$ and let $E \subseteq M$ be a porous set. Then $E$ has no points of density 1 and, in particular, $\mu(E)=0$.

Proof. Let $\alpha$ and $R$ be as in Definition A.7. Suppose by contradiction there exists $x \in E^{1}$. For every $r \in(0, R)$ there exists $y \in M$ such that $B(y, \alpha r) \subseteq B(x, r) \backslash E$. This implies that

$$
\frac{\mu(B(x, r) \backslash E)}{\mu(B(x, r))} \geq \frac{\mu(B(y, \alpha r))}{\mu(B(x, r))} \geq C,
$$

where $C>0$ depends on $\alpha$ and the doubling constant of $\mu$. Letting $r \rightarrow 0$ and taking into account that $x \in(M \backslash E)^{0}$, we get a contradiction. The last part of the statement follows from the generalized Lebesgue Theorem, see e.g. [28, Theorem 1.8].

Proposition A.9. Let $\left(\mathbb{R}^{n}, X\right)$ be a geodesic equiregular $C C$ space; then, for every $p \in \mathbb{R}^{n}$ and for every $r>0$ one has $\mathscr{L}^{n}(\partial B(p, r))=0$.

Proof. By Proposition A.8 it is sufficient to prove that $\partial B(p, r)$ is a porous set. Take $q \in$ $\partial B(p, r)$ and consider a length minimizing absolutely continuous path $\gamma:[0, r] \rightarrow \mathbb{R}^{n}$ such that $\gamma(0)=p, \gamma(r)=q$ and for every $t \in[0, r]$ one has $d(p, \gamma(t))=t$. Consider $\varepsilon \in(0,2 r]$ and set $y:=\gamma\left(r-\frac{\varepsilon}{2}\right) \in B(p, r)$. Then $B\left(y, \frac{\varepsilon}{2}\right) \subseteq B(q, \varepsilon)$, hence $B\left(y, \frac{\varepsilon}{2}\right) \cap \partial B(p, r)=\emptyset$, i.e., $\partial B(p, r)$ is porous.

\section{Appendix B. Proofs of some Results about jumps And approximate DIFFERENTIABILITY POINTS}

Proof of Proposition 2.28. (i) We can without loss of generality assume that $k=1$. Consider a countable dense subset $\left\{\left(a_{h}, b_{h}, \nu_{h}\right): h \in \mathbb{N}\right\}$ of $\mathbb{R} \times \mathbb{R} \times \mathbb{S}^{m-1}$ and, for every $h \in \mathbb{N}$, define $w_{h}: \mathbb{R}^{n} \rightarrow \mathbb{R}$ by

$$
w_{h}(y):= \begin{cases}a_{h} & \text { if } \widetilde{L}_{\nu_{h}}(y) \geq 0 \\ b_{h} & \text { if } \widetilde{L}_{\nu_{h}}(y)<0\end{cases}
$$

We first prove that (recalling the notation (7))

$$
\left(\Omega \backslash \mathcal{S}_{u}\right) \cup \mathcal{J}_{u}=\bigcap_{\ell=1}^{\infty} \bigcup_{h=0}^{\infty}\left\{p \in \Omega: \limsup _{r \rightarrow 0} f_{A(r)}\left|u \circ F_{p}-w_{h}\right| d \mathscr{L}^{n}<\frac{1}{\ell}\right\} .
$$

The inclusion $\subseteq$ in (48) is straightforward by Remark 2.20 and Proposition 2.26, In order to prove the opposite inclusion, consider $p \in \Omega$ such that for every $\ell \in \mathbb{N} \backslash\{0\}$ there exists $w_{h_{\ell}}$ such that

$$
\limsup _{r \rightarrow 0} f_{A(r)}\left|u \circ F_{p}-w_{h_{\ell}}\right| d \mathscr{L}^{n}<\frac{1}{\ell} .
$$


We prove that, possibly passing to a subsequence, there exist $a, b$ and $\nu$ such that $\left(w_{h_{\ell}}\right)$ is convergent in $L^{1}(A(1))$ to

$$
w(y):= \begin{cases}a & \text { if } \widetilde{L}_{\nu}(y) \geq 0 \\ b & \text { if } \widetilde{L}_{\nu}(y)<0\end{cases}
$$

Up to subsequences we can suppose that $\left(\nu_{h_{\ell}}\right)$ converges to some $\nu$. Define $C:=\mathscr{L}^{n}(A(1))$ and let $\bar{\ell} \in \mathbb{N}$ be such that for every $\ell, k \geq \bar{\ell}$ the set

$$
A^{+}(1):=\left\{y \in A(1): \widetilde{L}_{\nu_{h_{\ell}}}(y)>0 \text { and } \widetilde{L}_{\nu_{h_{k}}}(y)>0\right\}
$$

is such that $\mathscr{L}^{n}\left(A^{+}(1)\right) \geq \frac{1}{4} C$. By a change of variables, for such $h$ and $k$ one has

$$
\begin{aligned}
\left|a_{h_{\ell}}-a_{h_{k}}\right| & =f_{A^{+}(1)}\left|a_{h_{\ell}}-a_{h_{k}}\right| d \mathscr{L}^{n} \leq \frac{4}{C} \int_{A^{+}(1)}\left|w_{h_{\ell}}-w_{h_{k}}\right| d \mathscr{L}^{n} \\
& \leq \frac{4}{C} \int_{A(1)}\left|w_{h_{\ell}}-w_{h_{k}}\right| d \mathscr{L}^{n}=\frac{4}{C r} \int_{A(r)}\left|w_{h_{\ell}}-w_{h_{k}}\right| d \mathscr{L}^{n} \\
& \leq 4 f_{A(r)}\left|u \circ F_{p}-w_{h_{\ell}}\right| d \mathscr{L}^{n}+4 f_{A(r)}\left|u \circ F_{p}-w_{h_{k}}\right| d \mathscr{L}^{n} .
\end{aligned}
$$

Passing to the limsup as $r \rightarrow 0$ we get that $\left(a_{h_{\ell}}\right)$ is Cauchy and therefore convergent to some $a \in \mathbb{R}$. Using the same technique we also get that $\left(b_{h_{\ell}}\right)$ is convergent to some $b \in \mathbb{R}$, and $w_{h_{\ell}}$ converges in $L^{1}(A(1))$ to $w$. Now, for sufficiently large $\ell \in \mathbb{N}$ and for sufficiently small $r>0$, from

$$
\int_{A(r)}\left|u \circ F_{p} \circ \delta_{r}-w\right| d \mathscr{L}^{n} \leq \int_{A(r)}\left|u \circ F_{p} \circ \delta_{r}-w_{h_{\ell}}\right| d \mathscr{L}^{n}+\int_{A(1)}\left|w_{h_{\ell}}-w\right| d \mathscr{L}^{n}
$$

we deduce the remaining inclusion $\supseteq$ in (48).

Notice that the right-hand side of (48) is a Borel set if, for any $h \in \mathbb{N}$, and any small enough $r$, the function

$$
p \longmapsto f_{A(r)}\left|u \circ F_{p}-w_{h}\right| d \mathscr{L}^{n}
$$

is continuous. This is clearly true if $u$ is of class $C^{\infty}$. For general $u$, fix $p \in \Omega, r>0$ and $\varepsilon>0$ and consider $v \in C^{\infty}(\Omega)$ such that $\|u-v\|_{L^{1}\left(B\left(p, C_{1} r\right)\right)}<\varepsilon$, where $C_{1}$ is such that $F_{p}(A(r)) \Subset B\left(p, C_{1} r\right)$. Applying the triangular inequality, we find

$$
\begin{aligned}
& f_{A(r)}\left|u \circ F_{p}-u \circ F_{q}\right| d \mathscr{L}^{n} \\
\leq & f_{A(r)}\left(\left|u \circ F_{p}-v \circ F_{p}\right|+\left|v \circ F_{p}-v \circ F_{q}\right|+\left|v \circ F_{q}-u \circ F_{q}\right|\right) d \mathscr{L}^{n}<C \varepsilon,
\end{aligned}
$$

for some $C>0$, for every sufficiently small $r$ and for every $q$ sufficiently close to $p$. This proves that the function in (49) is continuous. It follows that $\left(\Omega \backslash \mathcal{S}_{u}\right) \cup \mathcal{J}_{u}$ is a Borel set: then, also $\mathcal{J}_{u}$ is a Borel set, for $\Omega \backslash \mathcal{S}_{u}$ is Borel and it is disjoint from $\mathcal{J}_{u}$.

Select now for any $p \in \mathcal{J}_{u}$ an $X$-jump triple $\left(u^{+}(p), u^{-}(p), \nu(p)\right)$ according to Definition 2.24, Define $\phi: \mathcal{J}_{u} \rightarrow \mathbb{R}^{m}$ by $\phi(p):=\left(u^{+}(p)-u^{-}(p)\right) \nu(p)$. We prove that $\phi$ is Borel, so that also $\nu$ is Borel up to re-defining it as $\nu(p)=\phi(p) /|\phi(p)|$. Set

$$
w_{p}(y):= \begin{cases}u^{+}(p) & \text { if } \widetilde{L}_{\nu(p)}(y)>0 \\ u^{-}(p) & \text { if } \widetilde{L}_{\nu(p)}(y)<0\end{cases}
$$


and

$$
\widetilde{A}(r):=\left\{y \in \mathbb{R}^{n}:\left|\left(y_{1}, \ldots, y_{m}\right)\right|+\sum_{j=m+1}^{n}\left|y_{j}\right|^{\frac{1}{w_{j}}} \leq r\right\} .
$$

Notice that the sets $\widetilde{A}(r)$ are invariant under rotations of the first $m$ coordinates. By Proposition 2.26 we have that for every $\psi \in C_{c}^{\infty}(\widetilde{A}(1))$ and every $i=1, \ldots, n$

$$
\begin{aligned}
\int_{\widetilde{A}(1)} w_{p} \partial_{i} \psi d \mathscr{L}^{n} & =\lim _{\varepsilon \rightarrow 0} \int_{\widetilde{A}(1)}\left(u \circ F_{p} \circ \delta_{\varepsilon}\right) \partial_{i} \psi d \mathscr{L}^{n} \\
& =\lim _{\varepsilon \rightarrow 0} \frac{1}{\varepsilon^{Q}} \int_{\widetilde{A}(\varepsilon)} u\left(F_{p}(y)\right) \partial_{i} \psi\left(\delta_{\varepsilon^{-1}}(y)\right) d \mathscr{L}^{n}(y) .
\end{aligned}
$$

Hence, for every $\psi \in C_{c}^{\infty}(\widetilde{A}(1))$ and for every $i=1, \ldots, n$ the function

$$
p \longmapsto \int_{\widetilde{A}(1)} w_{p} \partial_{i} \psi d \mathscr{L}^{n}
$$

is Borel. Fix $p \in \mathcal{J}_{u}$ and consider a sequence $\left(\psi_{h}\right)$ in $C_{c}^{\infty}(\widetilde{A}(1))$ converging to $\chi_{\widetilde{A}(1)}$. Computing the (Euclidean) measure derivative of $w_{p}$ we obtain that for every $i=1, \ldots, n$

$$
\begin{aligned}
& \phi^{i}(p) \mathscr{H}_{e}^{n-1}\left(\widetilde{A}(1) \cap\left\{\widetilde{L}_{\nu(p)}=0\right\}\right) \\
= & D^{i} w_{p}(\widetilde{A}(1))=\lim _{h} \int_{\widetilde{A}(1)} \psi_{h} d D^{i} w_{p}=-\lim _{h} \int_{\widetilde{A}(1)} w_{p} \partial_{i} \psi_{h} d \mathscr{L}^{n} .
\end{aligned}
$$

Since $\mathscr{H}_{e}^{n-1}\left(\widetilde{A}(1) \cap\left\{\widetilde{L}_{\nu(p)}=0\right\}\right)$ does not depend on $p$ we deduce by the previous step that $\phi$ is a Borel function, and therefore $\nu$ is Borel.

Finally, by Proposition 2.26 we have

$$
u^{+}(p)=\lim _{\varepsilon \rightarrow 0} \frac{1}{\varepsilon^{Q}} \int_{A(\varepsilon)} \chi_{\left\{\widetilde{L}_{\nu(p)}>0\right\}} u \circ F_{p} d \mathscr{L}^{n}
$$

and this concludes the proof.

The proof of (ii) is completely analogous to the Euclidean case, see [2].

Proof of Proposition 2.32. We can assume without loss of generality that $k=1$. Consider a dense subset $\left\{z_{i}: i \in \mathbb{N}\right\}$ of $\mathbb{R}^{m}$. Reasoning as in the proof of Proposition 2.28 one can prove that

$$
\mathcal{D}_{u}=\bigcap_{h=1}^{\infty} \bigcup_{i=0}^{\infty}\left\{p \in \Omega \backslash \mathcal{S}_{u}: \limsup _{\varrho \rightarrow 0} \frac{1}{r^{Q+1}} \int_{A(r)}\left|u \circ F_{p}-u^{\star}(p)-\widetilde{L}_{z_{i}}\right| d \mathscr{L}^{n}<\frac{1}{h}\right\}
$$

which implies that $\mathcal{D}_{u}$ is a Borel set.

We now prove that $D_{X}^{a p} u$ is Borel. Using Theorem 2.6, for any $p \in \mathcal{D}_{u}$ one has

$$
\lim _{\varepsilon \rightarrow 0} \frac{1}{\varepsilon^{Q+1}} \int_{\delta_{\varepsilon} P}\left|u \circ F_{p}-u^{\star}(p)-\widetilde{L}_{D_{X}^{a p} u(p)}\right| d \mathscr{L}^{n}=0
$$

where for every $n$-tuple of positive real numbers $\left(\ell_{1}, \ldots, \ell_{n}\right)$

$$
P=P\left(\ell_{1}, \ldots, \ell_{n}\right):=\left\{\xi \in \mathbb{R}^{n}: 0 \leq \xi_{j}^{1 / d_{j}} \leq \ell_{j} \text { for any } j=1, \ldots, n\right\}
$$

is the anisotropic box with axis that are parallel to the coordinate ones $\left(e_{1}, \ldots, e_{n}\right)$. By a change of variables we get

$$
\frac{1}{\mathscr{L}^{n}(P)} \int_{P} \widetilde{L}_{D_{X}^{a p} u(p)} d \mathscr{L}^{n}=\frac{1}{\mathscr{L}^{n}(P)} \lim _{\varepsilon \rightarrow 0} \frac{1}{\varepsilon^{Q+1}} \int_{\delta_{\varepsilon} P}\left(u \circ F_{p}-u^{\star}(p)\right) d \mathscr{L}^{n}
$$


From this we deduce that, for any $n$-tuple $\left(\ell_{1}, \ldots, \ell_{n}\right)$ the function

$$
p \longmapsto \frac{1}{\mathscr{L}^{n}(P)} \int_{P} \widetilde{L}_{D_{X}^{a p} u(p)} d \mathscr{L}^{n}
$$

is Borel. Now, for every $i=1, \ldots, m$ and every $h \in \mathbb{N} \backslash\{0\}$ define the rectangles $P_{h}^{i}:=$ $P(1 / h, \ldots, 1 / h, 1,1 / h, \ldots, 1 / h)$. A simple computation shows that

$$
\lim _{h} \frac{1}{\mathscr{L}^{n}\left(P_{h}^{i}\right)} \int_{P_{h}^{i}} \widetilde{L}_{D_{X}^{a p} u(p)} d \mathscr{L}^{n}=\frac{1}{2}\left(D_{X}^{a p} u(p)\right)_{i},
$$

which completes the proof.

Proof of Proposition 2.33. We can assume without loss of generality that $k=1$.

(i) By Remark 2.20, the functions $\widetilde{u}_{r}:=u \circ F_{p} \circ \delta_{r}$ and $\widetilde{v}_{r}:=u \circ F_{p} \circ \delta_{r}$ converge, respectively, to $u^{\star}(p)$ and $v^{\star}(p)$ in $L_{l o c}^{1}\left(\mathbb{R}^{n}\right)$ as $r \rightarrow 0$. In particular, as $r \rightarrow 0$ the families $\left(\widetilde{u}_{r}\right)$ and $\left(\widetilde{v}_{r}\right)$ converge (locally) in measure to $u^{\star}(p)$ and $v^{\star}(p)$ respectively. By a change of variables we have for any $R>0$

$$
\lim _{r \rightarrow 0} \mathscr{L}^{n}\left(\widehat{B}(0, R) \cap\left\{\widetilde{v}_{r} \neq \widetilde{u}_{r}\right\}\right)=\lim _{r \rightarrow 0} r^{-Q} \mathscr{L}^{n}\left(\widehat{B}(0, r R) \cap\left\{u \circ F_{p} \neq v \circ F_{p}\right\}\right)=0 .
$$

It follows that $\left(\widetilde{u}_{r}\right)$ and $\left(\widetilde{v}_{r}\right)$ have the same measure limit, hence $u^{\star}(p)=v^{\star}(p)$.

(ii) Using Proposition 2.26 and the same argument used in (i) we obtain that the functions

$$
U(y):=\left\{\begin{array}{ll}
u^{+}(p) & \text { if } \widetilde{L}_{\nu_{u}(p)}(y)>0 \\
u^{-}(p) & \text { if } \widetilde{L}_{\nu_{u}(p)}(y)<0
\end{array} \quad \text { and } \quad V(y):= \begin{cases}v^{+}(p) & \text { if } \widetilde{L}_{\nu_{v}(p)}(y)>0 \\
v^{-}(p) & \text { if } \widetilde{L}_{\nu_{v}(p)}(y)<0\end{cases}\right.
$$

coincide for $\mathscr{L}^{n}$-almost every $y$, hence $\left(u^{+}(p), u^{-}(p), \nu_{u}(p)\right) \equiv\left(v^{+}(p), v^{-}(p), \nu_{v}(p)\right)$.

(iii) By point (i) we already know that $u^{\star}(p)=v^{\star}(p)$. Since

$$
\frac{u\left(F_{p}\left(\delta_{r}(y)\right)\right)-u^{\star}(p)}{r} \neq \frac{v\left(F_{p}\left(\delta_{r}(y)\right)\right)-v^{\star}(p)}{r} \Longleftrightarrow u\left(F_{p}\left(\delta_{r}(y)\right)\right) \neq v\left(F_{p}\left(\delta_{r}(y)\right)\right),
$$

the statement follows using Proposition 2.31 and an argument similar to part (i) above.

\section{REFERENCES}

[1] Ambrosio, L. Some fine properties of sets of finite perimeter in Ahlfors regular metric measure spaces. Adv. Math. 159, 1 (2001), 51-67.

[2] Ambrosio, L., Fusco, N., And Pallara, D. Functions of bounded variation and free discontinuity problems. Oxford Mathematical Monographs. The Clarendon Press, Oxford University Press, New York, 2000.

[3] Ambrosio, L., Ghezzi, R., and Magnani, V. BV functions and sets of finite perimeter in subRiemannian manifolds. Ann. Inst. H. Poincaré Anal. Non Linéaire 32, 3 (2015), 489-517.

[4] Ambrosio, L., Kleiner, B., And Le Donne, E. Rectifiability of sets of finite perimeter in Carnot groups: existence of a tangent hyperplane. J. Geom. Anal. 19, 3 (2009), 509-540.

[5] Ambrosio, L., And Magnani, V. Weak differentiability of BV functions on stratified groups. Math. Z. 245, 1 (2003), 123-153.

[6] Ambrosio, L., And Scienza, M. Locality of the perimeter in Carnot groups and chain rule. Ann. Mat. Pura Appl. (4) 189, 4 (2010), 661-678.

[7] Bellaïche, A. The tangent space in sub-Riemannian geometry. In Sub-Riemannian geometry, vol. 144 of Progr. Math. Birkhäuser, Basel, 1996, pp. 1-78.

[8] Biroli, M., And Mosco, U. Sobolev and isoperimetric inequalities for Dirichlet forms on homogeneous spaces. Atti Accad. Naz. Lincei Cl. Sci. Fis. Mat. Natur. Rend. Lincei (9) Mat. Appl. 6, 1 (1995), 37-44.

[9] Bramanti, M., Miranda, Jr., M., and Pallara, D. Two characterization of BV functions on Carnot groups via the heat semigroup. Int. Math. Res. Not. IMRN, 17 (2012), 3845-3876.

[10] Capogna, L., Danielli, D., and Garofalo, N. The geometric Sobolev embedding for vector fields and the isoperimetric inequality. Comm. Anal. Geom. 2, 2 (1994), 203-215. 
[11] Comi, G. E., And Magnani, V. The Gauss-Green theorem in stratified groups. Preprint 2018, arXiv:1806.04011.

[12] Danielli, D., Garofalo, N., and Nhieu, D.-M. Trace inequalities for Carnot-Carathéodory spaces and applications. Ann. Scuola Norm. Sup. Pisa Cl. Sci. (4) 27, 2 (1998), 195-252 (1999).

[13] De Giorgi, E. Nuovi teoremi relativi alle misure $(r-1)$-dimensionali in uno spazio ad $r$ dimensioni. Ricerche Mat. 4 (1955), 95-113.

[14] Don, S., Le Donne, E., Moisala, T., And Vittone, D. In preparation.

[15] Don, S., Massaccesi, A., And Vittone, D. Rank-one theorem and subgraphs of BV functions in Carnot groups. Preprint 2017, arXiv:1712.02242.

[16] Don, S., And Vittone, D. A compactness result for BV functions in metric spaces. Ann. Acad. Sci. Fenn. Math., to appear. arXiv:1803.07545.

[17] Federer, H. Geometric measure theory. Die Grundlehren der mathematischen Wissenschaften, Band 153. Springer-Verlag New York Inc., New York, 1969.

[18] Folland, G. B., And Stein, E. M. Hardy spaces on homogeneous groups, vol. 28 of Mathematical Notes. Princeton University Press, Princeton, N.J.; University of Tokyo Press, Tokyo, 1982.

[19] Franchi, B., Gallot, S., and Wheeden, R. L. Sobolev and isoperimetric inequalities for degenerate metrics. Math. Ann. 300, 4 (1994), 557-571.

[20] Franchi, B., Serapioni, R., and Serra Cassano, F. Meyers-Serrin type theorems and relaxation of variational integrals depending on vector fields. Houston J. Math. 22, 4 (1996), 859-890.

[21] Franchi, B., Serapioni, R., and Serra Cassano, F. Approximation and imbedding theorems for weighted Sobolev spaces associated with Lipschitz continuous vector fields. Boll. Un. Mat. Ital. B (7) 11, 1 (1997), 83-117.

[22] Franchi, B., Serapioni, R., and Serra Cassano, F. Rectifiability and perimeter in the Heisenberg group. Math. Ann. 321, 3 (2001), 479-531.

[23] Franchi, B., Serapioni, R., and Serra Cassano, F. On the structure of finite perimeter sets in step 2 Carnot groups. J. Geom. Anal. 13, 3 (2003), 421-466.

[24] Garofalo, N., And Nhieu, D.-M. Isoperimetric and Sobolev inequalities for Carnot-Carathéodory spaces and the existence of minimal surfaces. Comm. Pure Appl. Math. 49, 10 (1996), 1081-1144.

[25] Garofalo, N., and Nhieu, D.-M. Lipschitz continuity, global smooth approximations and extension theorems for Sobolev functions in Carnot-Carathéodory spaces. J. Anal. Math. 74 (1998), 67-97.

[26] Gromov, M. Carnot-Carathéodory spaces seen from within. In Sub-Riemannian geometry, vol. 144 of Progr. Math. Birkhäuser, Basel, 1996, pp. 79-323.

[27] HajŁasz, P., And Koskela, P. Sobolev met Poincaré. Mem. Amer. Math. Soc. 145, 688 (2000), x+101.

[28] Heinonen, J. Lectures on analysis on metric spaces. Universitext. Springer-Verlag, New York, 2001.

[29] Jerison, D. The Poincaré inequality for vector fields satisfying Hörmander's condition. Duke Math. J. 53, 2 (1986), 503-523.

[30] Le Donne, E. Lecture notes on sub-Riemannian geometry. sites.google.com/site/enricoledonne/, 2017.

[31] Magnani, V. Elements of geometric measure theory on sub-Riemannian groups. Scuola Normale Superiore, Pisa, 2002.

[32] Magnani, V. Characteristic points, rectifiability and perimeter measure on stratified groups. J. Eur. Math. Soc. (JEMS) 8, 4 (2006), 585-609.

[33] Magnani, V., Tyson, J. T., and Vittone, D. On transversal submanifolds and their measure. J. Anal. Math. 125 (2015), 319-351.

[34] Magnani, V., And Vittone, D. An intrinsic measure for submanifolds in stratified groups. J. Reine Angew. Math. 619 (2008), 203-232.

[35] Marchi, M. Regularity of sets with constant intrinsic normal in a class of Carnot groups. Ann. Inst. Fourier (Grenoble) 64, 2 (2014), 429-455.

[36] Mitchell, J. On Carnot-Carathéodory metrics. J. Differential Geom. 21, 1 (1985), 35-45.

[37] Montgomery, R. A tour of subriemannian geometries, their geodesics and applications, vol. 91 of Mathematical Surveys and Monographs. American Mathematical Society, Providence, RI, 2002.

[38] Monti, R. Distances, boundaries and surface measures in Carnot-Carathéodory spaces, 2001. PhD thesis, cvgmt.sns.it/paper/3706/

[39] Monti, R., Pigati, A., And Vittone, D. On tangent cones to length minimizers in Carnot-Carathéodory spaces. SIAM J. Control Optim., to appear. cvgmt.sns.it/paper/3529/.

[40] Monti, R., And Serra Cassano, F. Surface measures in Carnot-Carathéodory spaces. Calc. Var. Partial Differential Equations 13, 3 (2001), 339-376. 
[41] Nagel, A., Stein, E. M., And Wainger, S. Balls and metrics defined by vector fields. I. Basic properties. Acta Math. 155, 1-2 (1985), 103-147.

[42] Rothschild, L. P., and Stein, E. M. Hypoelliptic differential operators and nilpotent groups. Acta Math. 137, 3-4 (1976), 247-320.

[43] Simon, L. Lectures on geometric measure theory, vol. 3 of Proceedings of the Centre for Mathematical Analysis, Australian National University. Australian National University, Centre for Mathematical Analysis, Canberra, 1983.

[44] Song, Y. Q., And Yang, X. P. BV functions in the Heisenberg group $H^{n}$. Chinese Ann. Math. Ser. A 24, 5 (2003), 541-554.

[45] Vittone, D. Regolarità delle geodetiche nei gruppi di Carnot. Master thesis, 2003. Available at cvgmt.sns.it/paper/972/.

[46] Vittone, D. Lipschitz surfaces, perimeter and trace theorems for BV functions in Carnot-Carathéodory spaces. Ann. Sc. Norm. Super. Pisa Cl. Sci. (5) 11, 4 (2012), 939-998.

(Don and Vittone) Università di Padova, Dipartimento di Matematica "T. Levi-Civita", via TrieSTe 63, 35121 Padova, Italy

E-mail address: don@math.unipd.it

E-mail address: vittone@math.unipd.it 